\title{
Etude quantitative sur le mariage précoce et le vécu des adolescentes dans la zone d'intervention du projet: Etat des lieux et perspectives
}

\author{
Gisele Kaboré \\ Population Council \\ Rene Dala \\ Aristide R. Bado
}

Follow this and additional works at: https://knowledgecommons.popcouncil.org/departments_sbsr-pgy

Part of the Demography, Population, and Ecology Commons, Family, Life Course, and Society Commons, Gender and Sexuality Commons, International Public Health Commons, and the Medicine and Health Commons How does access to this work benefit you? Let us know!

\section{Recommended Citation}

Kaboré, Gisele, Rene Dala, and Aristide R. Bado. 2009. "Etude quantitative sur le mariage précoce et le vécu des adolescentes dans la zone d'intervention du projet: Etat des lieux et perspectives," Rapport. Ouagadougou: Population Council. 
Projet : « Eliminer le mariage des enfants au Burkina : un plan pour la protection, le renforcement et l'action communautaire »

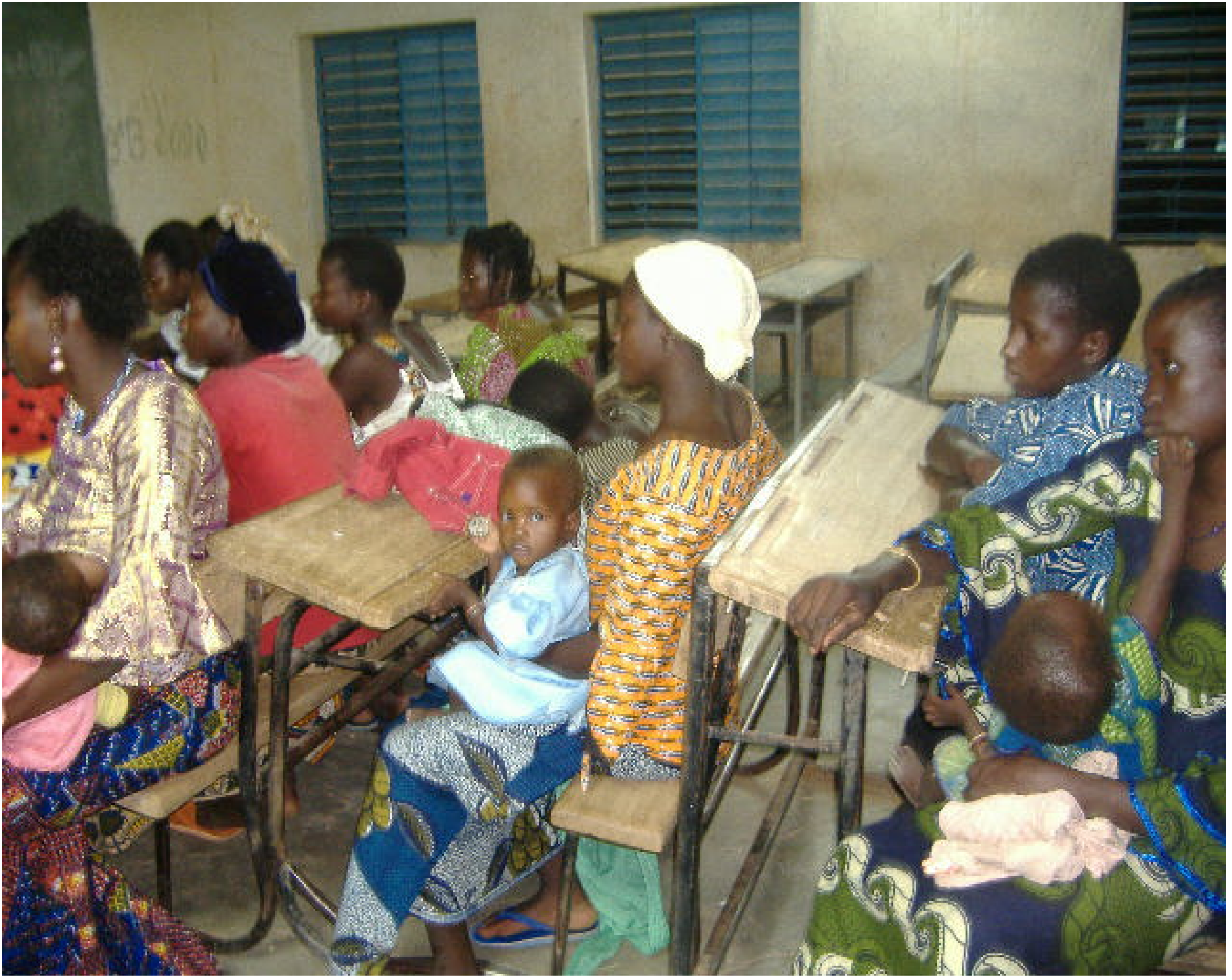

ETUDE QUANTITATIVE SUR LE MARIAGE PRECOCE ET LE VECU DES ADOLESCENTES DANS LA ZONE D'INTERVENTION DU PROJET : ETAT DES LIEUX ET PERSPECTIVES

(P Population Council 
PHOTOS DE COUVERTURE

PHOTO: Adolescentes mariées de Manga

EQUIPE DE RECHERCHE

Population Council

Gisèle Kaboré

Consultants

René Dala

Aristide R. Bado

EQUIPE DE TERRAIN

Superviseurs

Ouédraogo Yassia

Tall Yaya

Tioyé Justine

Zongo Lambert

Enquêteurs

Démé Rassoumane

Diallo Rahinatou

Illy Hawa

Kalmogo Flora

Kiénou Adama

Lompo Fidèle

Mano Daniel

Moyenga Hamidou

Pima Richard

Tilaté Kayaba

Zango Oussoumane 


\section{SOMMAIRE}

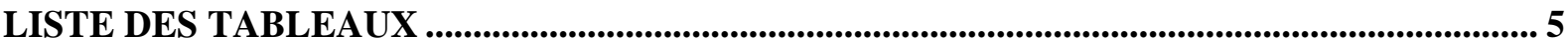

LISTE DES GRAPHIQUES........................................................................................................... 7

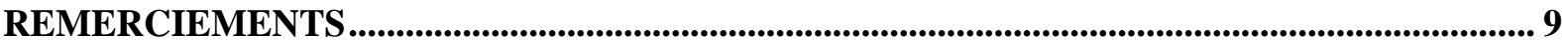

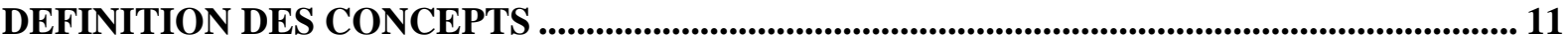

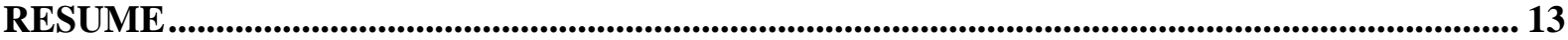

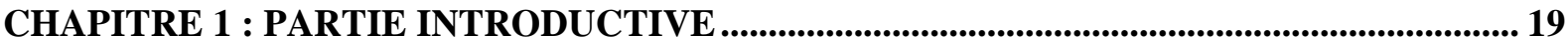

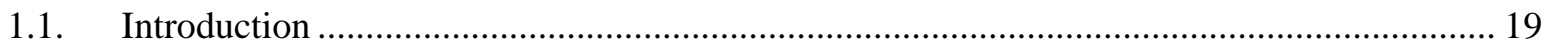

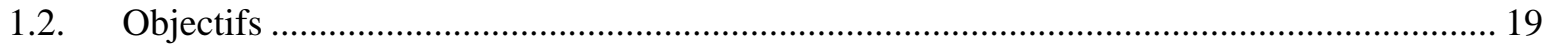

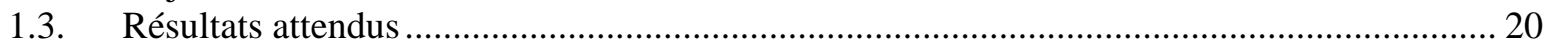

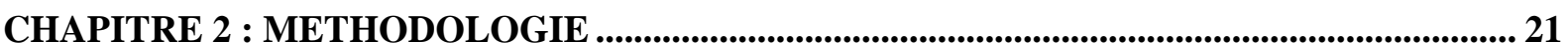

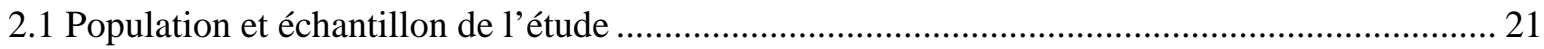

2.2 Sélection des ménages-échantillon et des adolescentes .............................................................. 22

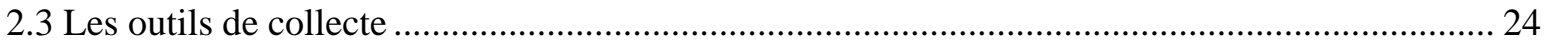

2.4 Recrutement, formation et sélection du personnel de terrain ...................................................... 24

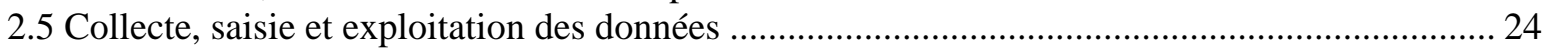

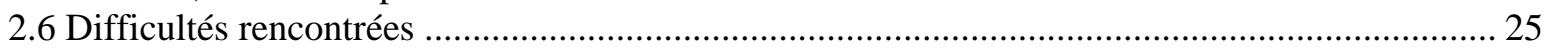

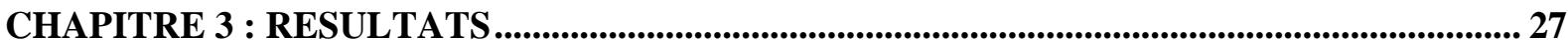

3.1 Caractéristiques sociodémographiques de la population étudiée .............................................. 27

3.1.1 Caractéristiques sociodémographiques des membres du ménage ........................................ 27

3.1.2 Caractéristiques sociodémographiques des chefs de ménage.......................................... 28

3.1.3 Caractéristiques sociodémographiques des adolescentes .................................................... 31

3.2 Connaissances des textes et de certaines dispositions légales concernant les adolescentes ......... 35

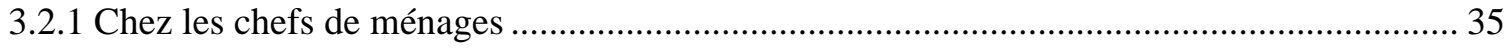

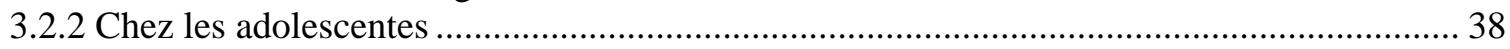

3.3. Perceptions sur le mariage des adolescents/tes............................................................................ 42

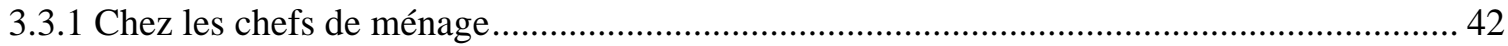

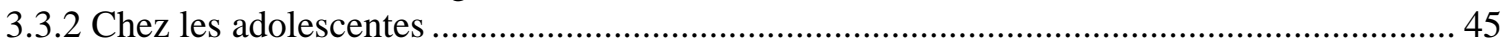

3.3.3 Canaux d'information chez les chefs de ménage ............................................................ 49

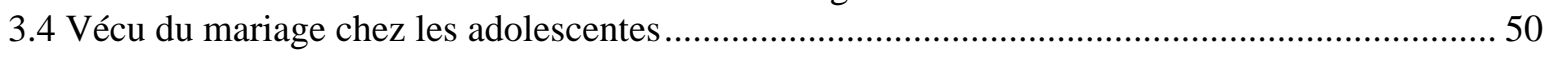

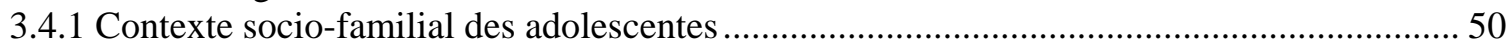

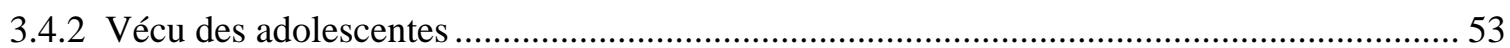

3.5 Connaissances, attitudes et pratiques en matière de la santé sexuelle et reproductive.....................60

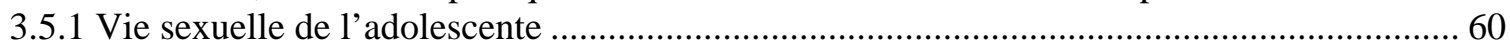

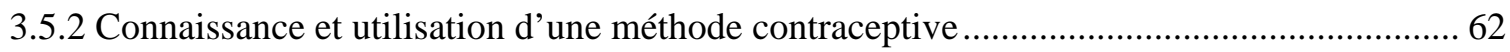

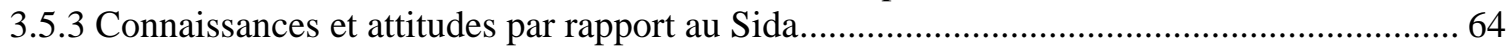

3.5.4 Niveau de connaissance des fistules obstétricales, des visites prénatales et postnatales..... 66

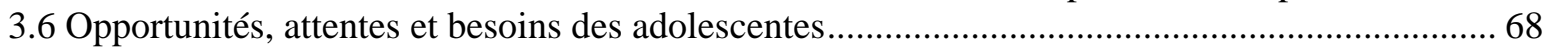

3.6.1 Opportunités, attentes et besoins en éducation et en formation................................................. 68

3.6.2 Opportunités, attentes et besoins en santé de la reproduction ................................................. 71

3.6.3 Opportunités, attentes et besoins d'emploi et d'accès au crédit ............................................ 74

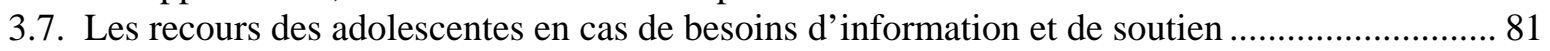

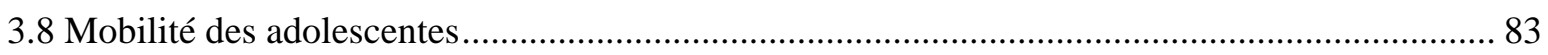

3.9 Relation entre le mariage précoce et certaines caractéristiques sociodémographiques et

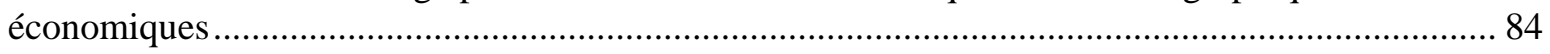

CHAPITRE 4 : CONCLUSION ET RECOMMANDATIONS ..................................................... 87

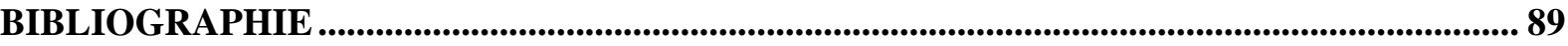

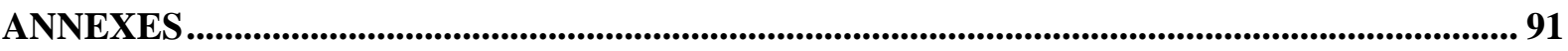

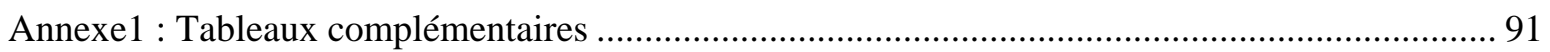





\section{LISTE DES TABLEAUX}

Tableau 2.1 : Répartition de l'échantillon entre les régions et entre les localités à l'intérieur des régions ............23

Tableau 3.1 : Structure par âge et sexe de la population recensée ..............................................................27

Tableau 3.2 : Répartition de la population selon le statut matrimonial et la région ..........................................28

Tableau 3.3 : Répartition par sexe des chefs de ménages enquêtés selon la région (\%) ....................................29

Tableau 3.4 : Répartition des chefs de ménage par niveau d’instruction selon la région (\%)..............................30

Tableau 3.5 : Répartition par région des chefs de ménage selon l’occupation principale (en \%) .........................31

Tableau 3.6 : Répartition des adolescentes par âge (en \%) ..........................................................................32

Tableau 3.7 : Répartition des adolescentes enquêtées selon l'ethnie (en \%)....................................................33

Tableau 3.8 : Répartition des adolescentes selon la religion (en \%) ................................................................33

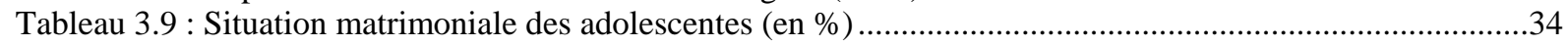

Tableau 3.10 : Niveau d'instruction des adolescentes (en \%) .....................................................................34

Tableau 3.11 : Connaissance des chefs de ménage de l’âge minimum légal au mariage (en \%) ..........................36

Tableau 3.12 : Connaissance de l'âge de scolarisation obligatoire selon la législation ......................................37

Tableau 3.13 : Connaissance des chefs de ménage de l’âge minimum légal pour un travail payé (en \%)............38

Tableau 3.14 : Age minimum pour le mariage de la fille et du garçon que prévoit le Code selon les déclarations des adolescentes

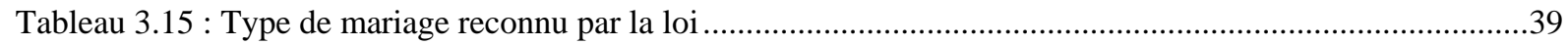

Tableau 3.16 : Connaissance des éléments constitutifs d’un dossier de mariage...............................................40

Tableau 3.17 : Connaissance du document officiel de mariage (en \%) ..........................................................40

Tableau 3.18 : Connaissance des conditions à remplir pour se marier légalement au Burkina Faso (en \%) .........41

Tableau 3.19 : Connaissance des conditions pour s’opposer à un mariage (en \%) ...........................................41

Tableau 3.20. Les structures de recours en cas de conflits conjugaux (en \%) ...............................................42

Tableau 3.21 : Age au mariage chez les filles selon la perception des chefs de ménage (en \%) .........................43

Tableau 3.22 : Age au mariage chez les garçons selon la perception des chefs de ménage (en \%) ......................43

Tableau 3.23 : Perception de la dot par les chefs de ménage (en \%) ...............................................................44

Tableau 3.24 : Opinions des chefs de ménage sur la scolarisation des filles et l'exercice d'une activité économique par la femme (en \%)................................................................................ 45

Tableau 3.25 : Perceptions des adolescentes sur l’âge auquel les filles et les garçons se marient généralement ou peuvent se marier dans les régions (en \%) ......................................................................... 46

Tableau 3.26 : Connaissances et perceptions par rapport à l’âge au mariage chez les adolescentes (en \%)..........46

Tableau 3.27: Caractéristiques de tendance centrale et de dispersion de l'âge au mariage précoce (en \%) ..........47

Tableau 3.28 : Pourcentage de mariage précoce (âge au premier mariage avant 17 ans) par région ....................47

Tableau 3.29 : Caractéristiques de tendance centrale et de dispersion de l’âge souhaité au mariage chez les adolescentes célibataires (en \%).

Tableau 3.30 : Caractéristiques de tendance centrale et de dispersion de l’âge souhaité au mariage chez les adolescentes mariées (en \%)................................................................................................ 48

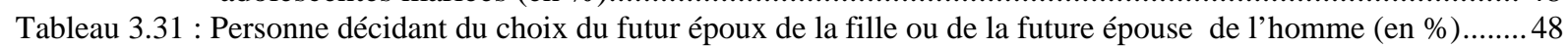

Tableau 3.32: Répartition des chefs de ménage selon le canal d’information le plus utilisé (en \%) ....................49

Tableau 3.33 : Possession d'équipement par les ménages (en \%) .................................................................50

Tableau 3.34 : Possession des moyens de transport (\%) ...........................................................................51

Tableau 3.35 : Possession d'électricité et la principale source d’approvisionnement en eau de boisson des ménages par région (en \%).

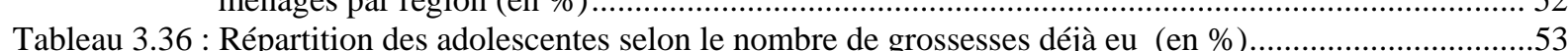

Tableau 3.37 : Choix du conjoint chez les adolescentes mariées (en \%) ...........................................................53

Tableau 3.38: Caractéristiques de tendance centrale et de dispersion de l’âge des adolescentes au premier mariage (en \%)

Tableau 3.39 : Type de cohabitation chez les adolescentes mariées (en \%)

Tableau 3.40 : Les conseillers des adolescentes sur la vie de couple (en \%) ......................................................56

Tableau 3.41 : Manquement du mari à ses devoirs d'époux (en \%) ......................................................................56

Tableau 3.42 : Personne assumant principalement les charges du ménage (en \%) ............................................57

Tableau 3.43: Prise de décisions dans les couples (en \%) ................................................................................58

Tableau 3.44 : Nature des conflits et mode de règlement (en \%) .......................................................................59

Tableau 3.45 : Recours en cas de besoins financiers dans les couples (en \%) ....................................................59

Tableau 3.46 : Thèmes de discussion dans les couples (en \%) ......................................................................60

Tableau 3.47 : Caractéristiques de tendance centrale et de dispersion de l'âge aux premiers rapports sexuels (années)

Tableau 3.48: Connaissance de la période féconde (en \%) .......................................................................61 
Tableau 3.49 : Connaissance de moyens pour éviter -une grossesse (en \%) ...................................................62

Tableau 3.50 : Sources d'information des adolescentes sur le planning familial (en \%) ...................................63

Tableau 3.51 : Les moyens par lesquels un individu peut contracter le Sida (en \%) ........................................64

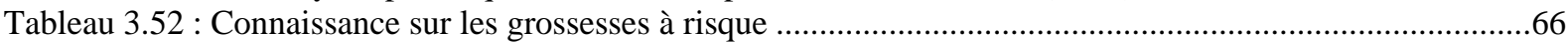

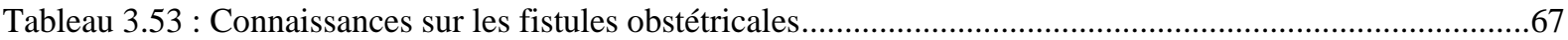

Tableau 3.54 : Connaissances des adolescentes sur les visites pré et postnatales ............................................67

Tableau 3.55 : Type de formation professionnelle reçus par les adolescentes enquêtées (en \%).........................69

Tableau 3.56 : Répartition des adolescentes selon le désir de poursuive une formation ou éducation plus poussée

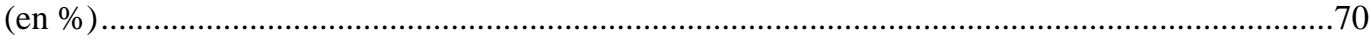

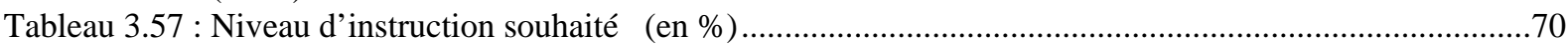

Tableau 3.58: Type de formation professionnelle souhaité par les adolescentes ...............................................70

Tableau 3.59 : Les opportunités en matière d’éducation existant dans les régions ..............................................71

Tableau 3.60 : Utilisation et niveau de satisfaction des centres de santé de la reproduction ................................72

Tableau 3.61 : Besoin des adolescentes en matière de santé de la reproduction................................................73

Tableau 3.62 : Activité principale des adolescentes (en \%) ..........................................................................75

Tableau 3.63 : Répartition des adolescentes selon l'exercice de travaux ménagers payants ...............................75

Tableau 3.64 : Activités économiques menées par les adolescentes (en \%) .....................................................75

Tableau 3.65 : Type de travail exercé par les adolescentes (en \%) ...................................................................76

Tableau 3.66 : Raisons évoquées par les adolescentes pour justifier leur travail (en \%) ....................................77

Tableau 3. 67 : Nature de l'emploi exercé par les adolescentes (en \%) .............................................................77

Tableau 3.68: Gestion des gains reçus par les adolescentes (en \%) .................................................................78

Tableau 3.69 : Utilisation de l'argent reçu par les adolescentes ....................................................................79

Tableau 3.70 : Répartition des adolescentes selon le secteur d'activités souhaité travailler (en \%) .....................79

Tableau 3.71 : Possibilité en emploi offerte par la localité (en \%) ..................................................................80

Tableau 3.72 -Appartenance ou non à des groupes de tontine et utilisation des ressources (en \%) .....................80

Tableau 3.73 : Présence d'une institution de micro-finances dans la localité et accès au crédit des adolescentes (en \%)

Tableau 3.74: Personnes ressources pour appartenir à une association (en \%) .................................................82

Tableau 75: Informations sur la mobilité des adolescentes.........................................................................83

Tableau 3.76 : Relation entre le mariage précoce et certaines caractéristiques sociodémographiques et économiques.

\section{Tableaux en annexe}

Tableau A1 : Lien de parenté des membres avec le chef de ménage ..............................................................91

Tableau A2 : Répartition par sexe des membres du ménage...........................................................................91

Tableau A3 : Nombre de chambres à coucher dans les ménages..................................................................91

Tableau A4 : Répartition des adolescentes selon le type d'école fréquentée (en \%) .........................................92

Tableau A5 : Indicateurs sur l'âge des conjoints des adolescentes (en \%) .......................................................99

Tableau A7 : Répartition des conjoints selon l’activité principale (en \%) ......................................................92

Tableau A8: Raisons pour l’âge souhaité au mariage chez les adolescentes ..................................................93

Tableau A10 : Caractéristiques de murs des logements par région (\%) .........................................................93

Tableau A11 : Caractéristiques de la toiture des logements par région (en \%)...................................................93

Tableau A12 : Type de toilettes des ménages par région.............................................................................93

Tableau A13 : Principal combustible utilisé pour la cuisine par région (en \%) ................................................94

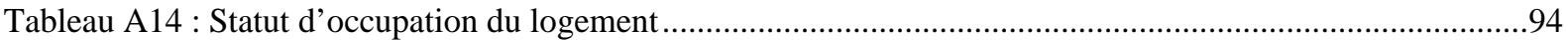

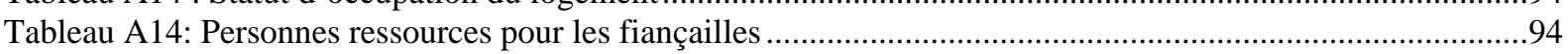

Tableau A15 : Personnes ressources pour sur les problèmes de santé ..............................................................95

Tableau A16: Personnes ressources pour les opportunités d'emploi .............................................................95

Tableau A17 : Personnes ressources pour la scolarisation ............................................................................95

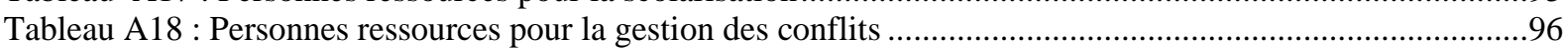

Tableau A19: Utilisation, type et lieu d'approvisionnement de méthodes contraceptives et les raisons de sa non utilisation chez les adolescentes

Tableau A20 : Connaissance d’un endroit où se procurer le préservatif..........................................................98

Tableau A21 : Présence d'un centre d'alphabétisation dans les localités ..........................................................98

Tableau A22 : Centre d'alphabétisation accessible aux adolescentes dans leur localité ......................................98

Tableau A23 : Présence de centres de formation professionnelle dans les localités ..........................................98

Tableau A24 : Type de formation professionnelle dans les localités ................................................................99

Tableau A25 : Fréquence des biens ou équipements possédés par les ménages selon le niveau de vie.................99 


\section{LISTE DES GRAPHIQUES}

Graphique 3.1 : Répartition de la population recensée selon le statut matrimonial et le sexe (en \%)................... 28

Graphique 3.2 : Répartition de chefs de ménage selon la fréquentation scolaire passée (\%)................................ 29

Graphique 3.3 : Répartition des chefs de ménage par région selon qu’ils soient alphabétisés ou non (\%)........... 30

Graphique 3.4: Répartition des chefs de ménages selon le milieu de résidence ................................................... 31

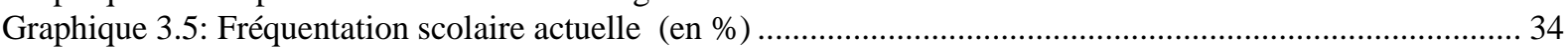

Graphique 3.6 : Répartition des adolescentes enquêtées selon le milieu de résidence (en \%) ................................. 35

Graphique 3.7 : Répartition des adolescentes selon le niveau du ménage ............................................................. 53

Graphique 3.8 : Choix du conjoint chez les adolescentes mariées selon le milieu de résidence (en \%) ............... 54

Graphique 3. 9: Utilisation du condom lors des premiers rapports sexuels ......................................................61

Graphique 3.10 : Répartition des adolescentes selon qu'elles aient entendu parler ou non du VIH (en \%) ......... 64

Graphique 3.11 : Formation professionnelle des adolescentes enquêtées (en \%) .................................................. 68

Graphique 3.12 : Répartition des adolescentes enquêtées selon qu'elles savent lire ou écrire (en \%)................... 69

\section{Graphique en annexe}

Graphique A1 : Présence de conflits dans les couples 



\section{REMERCIEMENTS}

L'équipe de recherche adresse ses remerciements à toutes les personnes qui ont contribué à la réalisation de cette étude. Nos remerciements vont particulièrement aux :

- partenaires techniques et financiers (UNFPA, UNICEF) et le Ministère de l'Action Sociale et de la Solidarité Nationale,

- responsables régionaux des régions du Centre-Sud, du Centre-Est, du Sahel, de l'Est et du Centre-Nord pour leur collaboration permanente,

- responsables provinciaux et départementaux qui ont facilité le travail de terrain,

- autorités administratives, locales et coutumières pour les facilités qu'elles ont apportées aux équipes sur le terrain,

- agents de terrain (contrôleurs, enquêteurs, agents de saisie) qui ont contribué de façon significative à la réussite de l’opération,

- populations des différentes localités visitées pour l’accueil réservé aux personnels de terrain et la disponibilité dont elles ont fait preuve. 



\section{DEFINITION DES CONCEPTS}

Le mariage : selon le Dictionnaire Robert, le mariage signifie l'union légitime entre un homme et une femme. Le mariage est une institution sociale et la légitimation de l'union entre des conjoints qui se soumettent à des obligations et à des droits réciproques.

La présente étude prend en compte toutes les formes d'union conjugale entre un homme et une femme. Il s'agit du mariage civil, du mariage coutumier, du mariage religieux et des unions libres.

Age légal au mariage de la fille : selon le Code des Personnes et de la Famille, l’âge légal au mariage de la fille est de 17 ans.

Mariage précoce : en se référant au Code des Personnes et de la Famille, la présente étude considère que le mariage est précoce chez l'adolescente lorsqu'il est contracté à moins de 17 ans.

Adolescente : Dans cette étude la définition de l'OMS a été retenue. Est considérée comme adolescente toute fille dont l'âge est compris entre 10 et 19 ans.

Adolescente mariée : dans cette étude, la notion d'adolescente mariée désigne donc toute fille dont l'âge est compris entre 10 et 19ans et vivant en union conjugale. Toutes les formes d'union sont prises en compte dans cette recherche.

Mobilité : dans cette étude, la notion de mobilité renvoie à la possibilité (avec ou sans permission) de l'adolescente de se rendre dans certains lieux tels que le marché, le centre de santé, les domiciles des voisins, etc.

La fistule : La fistule obstétricale est la constitution d'une communication anormale entre la vessie et le vagin (fistule vésico-vaginale) ou entre la vessie et le rectum (fistule vésicorectale). Elle survient d'ordinaire pendant un accouchement compliqué dû à l'étroitesse du bassin ou du rétrécissement de l'orifice vaginal et/ou vulvaire dans le cadre de l'excision. 



\section{RESUME}

L'étude sur le mariage précoce s'est déroulée dans cinq régions du Burkina Faso dont le Centre-Sud, l'Est, le Sahel, le Centre-Est et le Centre-Nord. Elle avait pour objectif général d'étudier les connaissances, les attitudes, les pratiques et les perceptions du mariage précoce ainsi que le vécu des adolescentes mariées ou non en vue d’orienter les actions du projet. L'étude a porté sur 1700 ménages et 1320 adolescentes âgées de 10 à 19 ans. La population totale recensée est de 10558 personnes dont 4992 hommes et 5566 femmes (52,7\%).Cette population est caractérisée par une forte proportion de jeunes. En effet les moins de 20 ans représentent $58 \%$ de la population totale. Ceci est valable pour toutes les régions. Plus de la moitié de la population âgée de plus de 10 ans est célibataire (52,4\%).

Les chefs de ménages sont surtout des hommes (89,4\%) contre 10,6\% pour les femmes chefs de ménage. Cette tendance s’observe dans toutes les régions. Une forte proportion d'entre eux n’a pas fréquenté l'école (52,6\%). Parmi ceux qui ont fréquenté, 45,4\% se sont limités au niveau primaire, $44,6 \%$ au niveau secondaire et seulement $8,7 \%$ au niveau supérieur. En terme d'occupation principale, la majorité des chefs de ménage (43,7\%) sont des agriculteurs. Parmi les femmes chefs de ménage, 9,2\% sont des adolescentes soit moins de $1 \%$ de l'ensemble des chefs de ménage.

L'âge moyen des adolescentes est de 15 ans contre 24 ans pour leur conjoint. Elles sont majoritairement des Mossi (60,7\%). Les Gourmantché représentent 12,9\%, les Bissa 10,8\% et les Peulhs 7,6\%. Plus de la moitié $(54,4 \%)$ sont des musulmanes, 36,1\% des catholiques, $8,4 \%$ sont des protestantes et seulement $1 \%$ des animistes. L'appartenance religieuse des adolescentes est du même ordre que celle des chefs de ménage.

La plupart des adolescentes sont célibataires (86,2\%). Les mariées représentent 9,3\% de l'ensemble. Concernant la fréquentation scolaire, 73,4\% d'entre elles ont été à l'école. Parmi celles-ci, plus de la moitié (51,4\%) ont atteint le niveau primaire et $48,1 \%$ le niveau secondaire. Chez les conjoints des adolescentes mariées, seulement $29 \%$ et $16 \%$ ont respectivement le niveau primaire et secondaire.

- S’agissant de la connaissance de certaines dispositions légales concernant le mariage, il ressort que :

-Chez les chefs de ménage : l'âge minimum au mariage pour la fille et le garçon tel que le prévoit le Code des Personnes et de la Famille du Burkina Faso (Burkina Faso, 1991), 17 ans pour la fille et 20 ans pour le garçon, est peu connu par les enquêtés. En effet, 46,1\% des chefs de ménage savent que la législation prévoit un âge minimum, $14,3 \%$ disent qu'il n'existe pas de législation en la matière et 39,6\% déclarent ne rien savoir. Parmi ceux qui ont déclaré connaître, très peu d'entre eux connaissent cet âge (25\% pour l'âge minimum au mariage des filles et $37,1 \%$ pour l'âge minimum au mariage des garçons).

Contrairement à l'âge minimum au mariage, une forte majorité des chefs de ménage connaissent que la législation prévoit un âge de scolarisation obligatoire des enfants (98,6\%). Parmi ceux-ci, 64,7\% ont déclaré que cet âge est de 7 ans contre 30,2\% qui ont cité l'âge de 6 ans.

-Concernant l'âge d'entrée sur le marché du travail, 66,6\% des chefs de ménage déclarent savoir que la législation prévoit un âge minimum légal pour un travail payé contre $19 \%$ d'entre eux qui ne le savent pas et 14,4\% qui déclarent qu'il n'y a aucune disposition en la matière. Cependant, très peu de chefs de ménage $(2,5 \%)$ connaissent que cet âge est fixé à 16 ans contre un peu plus du quart (26,3\%) qui fixent l’âge légal à la majorité (18 ans). 
- S'agissant des perceptions des chefs de ménage sur l'âge au mariage des adolescents/tes, on peut noter que le mariage des adolescentes est précoce dans les différentes régions concernées par l'enquête. En effet, 19\% des chefs de ménage déclarent que les adolescentes se marient avant 17 ans dans leurs localités et 10,6\% des chefs de ménage déclarent que les garçons se marient avant 20 ans.

- S'agissant de la perception et pratique de la dot chez les chefs de ménage, on peut conclure que la pratique de la dot est encore courante dans les localités. En effet, 90\% des chefs de ménage ont déclaré que la dot est encore pratiquée dans leurs localités. Cette pratique reste obligatoire selon 66,7\% des chefs de ménage. Parmi les chefs de ménages, $79,6 \%$ pensent que la pratique de la dot est une bonne chose contre respectivement $7,6 \%$ et $8,7 \%$ qui trouvent que c'est une mauvaise chose ou une pratique dépassée.

- S'agissant de certaines connaissances générales telles que l’importance de scolariser les filles d'une part et la possibilité qu'une femme exerce une activité économique d'autre part, on peut retenir que la quasi-totalité $(98,8 \%)$ des chefs de ménage pensent que c'est une bonne chose de scolariser les filles. Les raisons évoquées sont diverses : 61,6\% pensent que c'est un droit humain. Les pourcentages de déclaration des autres raisons telles que le pouvoir de se prendre en charge, le meilleur encadrement des enfants, la contribution aux dépenses du ménage et pour une meilleure réceptivité des messages de sensibilisation, tournent autour de 30\%. Pour ce qui est de l'exercice d'une activité économique par la femme, 92,3\% des chefs de ménage pensent que la femme peut exercer librement une activité économique.

- Concernant le canal d'information des chefs de ménage sur les événements du pays et du monde, il ressort que la radio est le canal le plus utilisé (50,9\%). Ensuite suivent les causeries familiales $(23,8 \%)$ et la télévision $(23,7 \%)$.

- S'agissant des perceptions des adolescentes sur l'âge auquel les filles et les garçons se marient généralement ou peuvent se marier, on peut souligner que selon 27,4\% des déclarations des adolescentes, les filles se marient généralement à 17 ans et selon $40 \%$ de leurs déclarations, les garçons se marient à 20 ans. L'âge à partir duquel une fille peut se marier est de 20 ans selon 30\% des déclarations des adolescentes contre 25 ans pour les garçons $(32,1 \%)$.

- Concernant les connaissances sur le Code des personnes et de la famille, le constat est que parmi les adolescentes interrogées, 91,8\% déclarent n’avoir pas entendu parler du Code ou ne savent pas. Parmi celles qui en ont entendu parler (8,3\%), 65,8\% déclarent savoir que le Code prévoit un âge minimum au mariage pour les filles et pour les garçons. Cependant cet âge est mal connu par les adolescentes. Dans le cas de la fille, le pourcentage de bonnes réponses est de $24,6 \%$ contre $36,2 \%$ pour le garçon. D’une manière générale, moins de la moitié des adolescentes $(48,5 \%)$ pensent que l'âge au mariage de la fille selon le Code est acceptable tandis que 56,4\% pensent que l'âge au mariage du garçon selon le Code est acceptable. 
Le contenu du Code des personnes et de la famille est très peu connu par les adolescentes. Seulement 39,1\% des adolescentes ont pu identifier le mariage civil comme étant celui reconnu par la loi.

De même les éléments constitutifs d'un dossier de mariage ne sont pas maîtrisés.

Par ailleurs, la grande majorité des adolescentes enquêtées (72\%) ne savent pas par quel document officiel on peut reconnaître que le mariage a été célébré. Seulement, 25\% des adolescentes citent le certificat de mariage comme document officiel d'acte de mariage.

Il ressort que d'une manière générale, les conditions pour se marier légalement au Burkina Faso ne sont pas bien maîtrisées par les adolescentes.

Dans l'ensemble, $11,8 \%$ des adolescentes déclarent « que les deux conjoints doivent être obligatoirement de sexe différent », 11,6\% citent l'âge obligatoire selon le Code, 22,6\% citent le consentement des 2 conjoints.

D’une manière générale, les conditions d'opposition au mariage sont mal connues par les adolescentes. Environ 30\% citent comme conditions l'absence de consentement des 2 conjoints, l'absence de consentement des parents.

Environ 50\% des adolescentes déclarent connaître de structures officielles auxquelles elles peuvent recourir en cas de conflits conjugaux, mais lorsqu'il s'agit de les citer, c'est surtout l'Action sociale qui revient fréquemment. Les autres adolescentes ne connaissent aucune structure et affirment se référer souvent aux parents pour régler ces conflits.

- S'agissant des perceptions du mariage chez les adolescentes, des analyses il ressort que la moitié des adolescentes déclarent que pour les filles, un mariage avant 15 ans peut être considéré comme un mariage précoce. Cependant, en considérant l’âge de 17 ans prescrit dans le Code, l'ampleur du mariage précoce est de 37,2\% dans la zone d'étude.

Pour le choix du conjoint, 67,5\% des adolescentes déclarent que c'est la jeune fille elle-même qui doit choisir son conjoint, et pour le choix de la future épouse, 71,1\% d'entre elles affirment que c'est le jeune homme qui doit décider lui-même. Cependant, chez les adolescentes mariées, seulement 34,3\% déclarent qu'elles ont fait le choix de leur conjoint sans l'influence de la famille (37\% en milieu urbain contre $30,6 \%$ en milieu rural).

- Concernant le vécu du mariage chez les adolescentes, deux aspects ont été analysés :

\section{Le contexte socio-économique et le vécu du mariage.}

Le contexte socio-économique peut être décrit par certaines caractéristiques de l'équipement et de l'habitat des ménages. Ces caractéristiques éclairent sur les conditions de vie et de santé des ménages qui peuvent influencer les pratiques, attitudes et perceptions sur le mariage précoce et le vécu des adolescentes.

En terme d'équipement, sur l'ensemble des ménages enquêtés, 82\% possèdent une radio. Seulement $39,5 \%$ et $12,2 \%$ d'entre eux possèdent respectivement un poste téléviseur et un réfrigérateur. Près de deux tiers $(65,5 \%)$ de ménages possèdent un téléphone (fixe ou mobile).

En terme de moyen de transport, le vélo est le moyen de transport le plus fréquent chez les ménages (83,5\%). Viennent ensuite la mobylette (36\%) et la moto $(22,3 \%)$. Seulement une proportion très réduite de ménages $(3,1 \%)$ possèdent une voiture. Le vélo est le moyen de transport le plus utilisé (54,5\%).

Concernant les caractéristiques des logements, il ressort que $42,8 \%$ des ménages possèdent l'électricité, 48,4\% utilisent l'eau des robinets publics ou l'eau courante à domicile.

Le bois est le principal combustible utilisé pour faire la cuisine dans les ménages (86,5\%). 
Les murs des logements sont le plus souvent en banco (35,3\%) ou en banco amélioré (37,3\%) et plus rarement en ciment (26,3\%). La toiture est dans la majorité des cas en tôles ondulées (72,5\%). Trois ménages sur 10 (29,6\%) ne possèdent pas de toilettes dans leur logement.

En plus de la description des caractéristiques de l'équipement et de l'habitat, un proxy de niveau de vie des ménages des adolescentes a été construit. Il ressort que 69,5\% des adolescentes sont issues de ménages à faible niveau de vie, $27,1 \%$ sont issues de ménages dont le niveau de vie est moyen et 3,4\% sont issues de ménages dont le niveau de vie est élevé.

La mise en relation entre le mariage précoce et certaines caractéristiques sociodémographiques et économiques telles que le milieu de résidence, la région, le niveau de vie, la fréquentation scolaire passée, le niveau d'instruction, la taille du ménage, la religion et l'ethnie fait ressortir qu'il existe un lien entre la région, le niveau de vie, la fréquentation scolaire passée, la religion et l'ethnie.

Concernant le vécu du mariage, on peut retenir les points suivants :

Par rapport à l'âge souhaité au mariage, $50 \%$ des adolescentes célibataires souhaiteraient se marier après 20 ans et 50\% des adolescentes mariées auraient souhaité se marier après 19 ans. Par rapport à l'âge au mariage, 50\% des adolescentes se sont mariées avant 17 ans. Parmi elles, 73\% trouvent que leur âge au mariage est convenable. Plus de $50 \%$ des conjoints des adolescentes se sont mariés après 26 ans. L’âge moyen au premier mariage chez les adolescentes est 16,6 ans tandis que chez leurs conjoints, l'âge moyen au mariage est de 27,2 ans. Les adolescentes se marient à des conjoints plus âgés qu'elles (environ 10 ans).

En ce qui concerne les grossesses, 10,6\% des adolescentes ont déjà eu au moins une grossesse.

Les adolescentes reçoivent des conseils dans plusieurs domaines. Sur la vie en couple, la mère est la personne centrale $(81,6 \%)$. Sur les fiançailles et le mariage la mère constitue la personne centrale (22\%), suivie des amis $(17,4 \%)$ et du père $(14,6 \%)$. Sur la santé, c'est principalement le père $(28,7 \%)$ et ensuite la mère $(21 \%)$ qui donnent les informations. En matière d'emploi, le père est le principal conseiller (22,4\%). S'agissant des opportunités de scolarisation, de la gestion des conflits, c'est essentiellement la mère qui donne les conseils (23\% de déclarations dans chaque cas).

En cas de conflit dans le couple, aucun recours n’est sollicité, selon $25 \%$ des déclarations des adolescentes. Dans la majorité des cas (52,5\%), le recours est fait aux parents du mari. La justice ou les associations sont peu sollicitées.

- Les connaissances des adolescentes sur les grossesses à risque et les fistules obstétricales sont insuffisantes. En effet, 59,4\% d'entre elles déclarent ne pas connaître les signes d'une grossesse à risque et moins de $10 \%$ déclarent avoir entendu parler de fistules obstétricales.

- L'importance des visites prénatales et postnatales est bien connue par les adolescentes. Plus de 9 adolescentes sur 10 (94\%) savent que ces visites doivent être effectuées. Cependant, seulement $17 \%$ d'entre elles savent que quatre visites prénatales sont recommandées au cours d'une grossesse.

- Au regard de la vie sexuelle de l'adolescente, $29 \%$ des adolescentes sont sexuellement actives et parmi elles, 54,1\% n'ont pas utilisé de condom lors de leurs premiers rapports sexuels. On note aussi que 60\% des adolescentes n’ont pas entendu parler de période féconde. 
- Quant à la connaissance et l'utilisation d'une méthode contraceptive, 42,7\% des adolescentes déclarent connaître des moyens pour éviter d'avoir une grossesse. Les moyens cités sont principalement le condom (73,1\%), la pilule (53\%), les injections (36,3\%) et l'abstinence (31,8\%). Parmi les adolescentes qui ont déclaré connaître une méthode pour éviter d'avoir une grossesse, 43,3\% déclarent en utiliser. Le condom est la méthode la plus utilisée (66,5\%).

- Concernant le planning familial, 47,7\% des adolescentes en ont entendu parler. Les canaux d'informations cités sont principalement la télévision (31,4\%), les amis (24,2\%), radio (23,7\%), les réunions (23,7\%) et les parents (10,5\%).

- Par rapport au Sida, 86,3\% des adolescentes en ont entendu parler. Les relations sexuelles non protégées (72\%), le partage de seringue (39,5\%), la transfusion sanguine $(19,3 \%)$ sont les voies de transmission du Sida les plus connues par les adolescentes. La transmission mère-enfant est très peu connue $(7,4 \%)$.

- En terme d'opportunités, d'attentes et de besoins des adolescentes, on peut retenir les points suivants :

-en éducation et formation professionnelle : seulement $7,4 \%$ des adolescentes ont reçu une formation professionnelle. Parmi celles qui ne sont pas actuellement à l'école, $80,8 \%$ souhaiteraient bénéficier d'une éducation ou d'une formation plus poussée.

Les besoins en formation professionnelle sont exprimés dans des domaines tels que le tissage/ teinture/ couture/ broderie $(27,7 \%)$, la santé $(11,8 \%)$, la gestion ou le commerce $(10,3 \%)$ et dans une moindre mesure en informatique $(8,3 \%)$, en alphabétisation (6,4\%), en secrétariat (4\%) et en coiffure (4\%). 89\% des adolescentes seraient disponibles à participer dans des programmes visant à améliorer les domaines de l'éducation, de la santé et de l'emploi.

-en santé de la reproduction : très peu d'adolescentes ont déjà utilisé des centres de santé de la reproduction (26,7\%). Parmi elles, 82\% déclarent avoir été satisfaites de ces services.

Les besoins en santé de la reproduction sont de deux ordres. En matière d'offre de services, les améliorations suivantes ont été sollicitées par les adolescentes: la proximité du centre de santé, l'accessibilité des médicaments, la disponibilité en personnel qualifié et en nombre suffisant et des services appropriés en santé de la reproduction des adolescentes (planning familial, vaccination, centre d'écoute pour les jeunes, counselling, sensibilisation). En matière d'information, les adolescentes aimeraient avoir davantage d'informations sur les IST, sur le planning familial et sur les soins prénatals et postnatals.

-en terme d'emploi et accès au crédit : on peut noter que $56,1 \%$ des adolescentes sont des élèves; 20,8\% exercent les travaux domestiques, 6,9\% sont dans le commerce. 11,4\% des adolescentes déclarent avoir déjà exercé une activité génératrice de revenus. 59,2\% des adolescentes ont commencé une activité génératrice de revenu à moins de 15 ans. Leurs motivations pour le travail sont le besoin d'avoir de l'argent (4,3\%), la recherche de l'indépendance financière (26,2\%), la fuite devant l'oisiveté (26,2\%), la contribution au revenu familial (14,3\%). 
L’accès au crédit n’est pas une réalité fréquente chez les adolescentes. En effet, 5\% d'entre elles ont déclaré qu'elles font partie d'une tontine et $0,8 \%$ ont eu recours à une institution de micro-finance.

Au regard des résultats qui découlent de l'étude, les recommandations suivantes peuvent être formulées afin de contribuer à améliorer la situation des adolescentes et à réduire l'ampleur du mariage précoce dans la zone d'étude :

- sensibiliser davantage les parents, l'entourage et les adolescentes sur les inconvénients du mariage précoce ;

- sensibiliser les chefs de ménage, l'entourage et les adolescentes sur le Code des personnes et de la famille ;

- appuyer financièrement les adolescentes qui souhaitent fréquenter ou poursuivre leurs études et créer ou renforcer des structures d'accueil pour des formations professionnelles accessibles aux adolescentes ;

- renforcer la connaissance des adolescentes sur la santé sexuelle et reproductive et mettre à leur disposition des services appropriés ;

- faciliter l'accès des adolescentes aux crédits, les sensibiliser sur les activités génératrices de revenus et renforcer leurs capacités en matière de gestion. 


\subsection{Introduction}

Le mariage précoce est un phénomène courant en Afrique Sub-saharienne. Le Burkina Faso ne fait pas exception. En effet, d'après l'EDS 2003, l'âge médian d'entrée en première union des femmes de 25-49 ans est estimé à 17,7 ans. La pratique est plus répandue en milieu rural qu'en milieu urbain. Au Burkina Faso, 63\% des filles issues de milieux ruraux étaient mariées ou en concubinage avant 18 ans contre 22\% en milieux urbains (EDS, 2003). Malgré les raisons avancées par les parents, le mariage précoce peut avoir des conséquences préjudiciables pour le développement des enfants se trouvant dans cette situation. On peut noter entre autres, le refus d'aller à l'école, les problèmes de santé maternelle et infantile liés aux grossesses prématurées, les infections sexuellement transmissibles, y compris le VIH/SIDA, la maltraitance, etc.

Malgré l'ampleur du phénomène, très peu de données sont disponibles sur le mariage précoce et les aspects juridiques et psychosociaux sont rarement pris en compte. Au-delà des efforts consentis par l'Etat et ses partenaires beaucoup reste encore à faire pour combler le manque de données nécessaires pour mieux appréhender le mariage précoce dans toutes ses dimensions d'une part, et d'autre part pour fournir une base fiable permettant de développer des interventions et stratégies pour satisfaire les besoins des adolescentes et pour éliminer la pratique du mariage précoce.

C'est pour contribuer à apporter des solutions aux problèmes que vivent les adolescentes que l'UNICEF, l'UNFPA, le Population Council et le Ministère de l'Action Sociale ont décidé d'intervenir dans cinq régions où le phénomène est d'une certaine ampleur.

La présente étude permettra de mieux comprendre la situation réelle des adolescentes et de recenser leurs besoins en éducation/formation, en santé sexuelle et reproductive et sur le plan économique.

\subsection{Objectifs}

L’objectif général de cette étude est de :

\section{Objectif général :}

Etudier les connaissances, les attitudes, les pratiques et les perceptions du mariage précoce ainsi que le vécu des adolescentes mariées ou non en vue d’orienter les actions du projet.

\section{Objectifs spécifiques :}

Pour atteindre cet objectif général, il s’agit de :

- Déterminer le profil sociodémographique et économique des adolescentes, de leurs conjoints et de leurs ménages ;

- Faire un état des lieux sur les connaissances, perceptions, attitudes et pratiques des adolescentes et des chefs de ménage en matière de mariage dans la zone d'intervention du projet.

- Décrire le contexte social et familial des adolescentes et le vécu des adolescentes mariées.

- Evaluer les besoins des adolescentes en termes d'opportunités économiques, d'éducation et de santé de la reproduction ;

- Faire des recommandations en vue de réduire l'ampleur du mariage précoce dans la zone d’intervention du projet. 


\subsection{Résultats attendus}

Les résultats attendus pour cette étude sont :

$\checkmark$ Le profil sociodémographique et économique des adolescentes, de leurs conjoints et de leurs ménages est connu ;

$\checkmark$ L’ampleur du mariage précoce est déterminée dans la zone d’intervention du projet ;

$\checkmark$ Un état des lieux sur les connaissances, attitudes, pratiques et perceptions en matière de mariage au Burkina Faso est fait ;

$\checkmark$ Le contexte social et familial et le vécu des adolescentes victimes de mariage précoce est décrit ;

$\checkmark$ Les besoins des adolescentes en termes d'opportunités économiques, d'éducation et de santé sexuelle et de la reproduction sont identifiés ;

$\checkmark$ Des recommandations sont faites pour orienter les actions du projet. 


\subsection{Population et échantillon de l'étude}

La zone d'étude comprend cinq régions à savoir le Centre-Sud, l'Est, le Sahel, le Centre-Est et le Centre-Nord. Elles ont été choisies en fonction de l'ampleur du mariage précoce.

La population d'étude est constituée par l'ensemble des adolescentes âgées de 10 à 19 ans au moment de l'enquête et qui résident dans la zone d'étude composée de l'ensemble des 24 localités retenues dans les cinq régions.

Toutes ces localités ont été retenues compte tenu du type d'échantillonnage opté pour l'étude (sondage stratifié proportionnel).

L'échantillon d'enquête a été tiré de façon aléatoire à partir de cette population. A cet effet, chaque région a été considérée comme une strate. Chaque strate est définie par l'ensemble des localités qui la composent. La taille de la strate est la population totale de ces localités. Il a été procédé à un sondage stratifié proportionnel : la taille globale de l'échantillon d'étude a été déterminée dans un premier temps et a été répartie proportionnellement au poids de chaque strate.

Pour ce faire, les étapes suivantes ont été adoptées pour déterminer la taille de l'échantillon d'étude ${ }^{1}$ :

- Estimation du pourcentage de mariages précoces dans la zone d’étude : 20\% ;

- Choix du changement attendu à la fin du projet, exprimé en points pourcentage : 10 points pourcentage ;

- Choix de la précision nécessaire pour détecter le changement attendu : cette précision est égale à la moitié du changement en points pourcentage, soit $\pm 5 \%$;

- Choix du dénominateur approprié pour le pourcentage de mariages précoces: les adolescentes âgées de 10 à 19 ans; ce dénominateur a été obtenu en consultant la Table générée à cet effet en intégrant le changement attendu et la précision.

Avec un changement de 10 points pourcentage et une précision égale à $\pm 5 \%$, la Table donne 1290 adolescentes de 10 à 19 ans.

- détermination du facteur de conversion nécessaire pour convertir le dénominateur taille de l'échantillon (nombre d'adolescentes âgées de 10 à 19 ans) en taille d'échantillon (nombre de ménages à enquêter). En d'autres termes, quel est le nombre moyen de ménages nécessaire pour trouver une adolescente âgée de 10 à 19 ans? Il a été estimé que dans approximativement 75\% des ménages, on peut trouver une adolescente âgée de 10 à 19 ans, ce qui a conduit à un facteur de conversion égal à 1/0,75, soit 1,33. En multipliant la taille

\footnotetext{
1 La source de cette table est : Household Survey Manual : Diarrhoea Case Management, Morbidity, and Mortality, Diarrhoeal Disease Control Programme, World Health Organisation, Geneva, 1989.
} 
d'échantillon (nombre d'adolescentes) par 1,33 (ou en la divisant par 0,75) on a obtenu le nombre de ménages-échantillon à interroger, soit 1720 ménages.

- à l'intérieur de chaque strate, l'échantillon a été réparti entre les localités, proportionnellement à leur poids dans la strate (pourcentage de leur population par rapport à la population totale de la strate).

- les populations utilisées pour la détermination de la taille de l'échantillon sont basées sur les données du recensement général de la population et de l'habitation de 2006 pour les sites concernés.

\subsection{Sélection des ménages-échantillon et des adolescentes}

En l'absence d'une base de sondage à jour (liste complète des ménages par localité et par strate ainsi que la population à jour des strates et localités), les ménages ont été sélectionnés par tirage aléatoire systématique selon les instructions suivantes fournies aux superviseurs : en se plaçant dans une position centrale dans chaque localité, à l'intérieur de chaque strate, inscrire les noms des 4 points cardinaux (Nord, Sud, Est, Ouest) sur des bouts de papier qui doivent être mélangés et mis dans un carton ; demander à une tierce-personne de tirer au hasard un bout de papier qui indiquera la direction dans laquelle les enquêteurs doivent s'orienter pour le tirage des ménages. A partir de cette direction, suivre un axe sur lequel il faut compter le nombre de concessions ; sélectionner ensuite au hasard un nombre compris entre 1 et le nombre de concessions sur l'axe. Ce numéro de concession sera le point de départ de l'enquête. Ensuite les concessions ont été sélectionnées de manière aléatoire systématique à raison d'une concession sur deux. A l'intérieur de chaque concession, un ménage sur deux a été sélectionné.

Dans les ménages tirés et après l'introduction faite au chef de ménage et après avoir obtenu son consentement tous les membres du ménage ont été enregistrés, ce qui a permis d'identifier les adolescentes éligibles (10-19 ans. Les formulaires de consentement signés sont gardés dans les archives. S’il n’y a pas d'adolescente éligible dans un ménage tiré, on progresse dans la sélection en suivant toujours le même procédé. Si dans un même ménage, il y a plusieurs adolescentes éligibles, il a été convenu de les enquêter toutes si elles ne dépassent pas 3 et au cas échéant d'enquêter au maximum 3, tout en gardant le souci d'obtenir l'échantillon prévu. Une fois la sélection terminée sur l'axe, les enquêteurs ont évolué selon le sens des aiguilles d'une montre pour sélectionner les autres ménages jusqu' à l'obtention du nombre total de ménages et concomitamment du nombre total d'adolescentes. La répartition de l'échantillon entre les localités se trouve dans le tableau ci-dessous (tableau 2.1). 
Tableau 2.1 : Répartition de l'échantillon entre les régions et entre les localités à l'intérieur des régions

\begin{tabular}{|c|c|c|c|}
\hline $\begin{array}{l}\text { Région du CENTRE-SUD : } \\
396 \text { ménages ; } \\
297 \text { adolescentes 10-19 ans }\end{array}$ & $\begin{array}{l}\text { Nombre de ménages } \\
\text { à enquêter }\end{array}$ & $\begin{array}{l}+10 \% \\
\text { (remplacement) }\end{array}$ & $\begin{array}{l}\text { Nombre d'adolescentes } 10-19 \text { ans à } \\
\text { enquêter }\end{array}$ \\
\hline 1. Manga & 87 & 96 & 65 \\
\hline 2. Kombissiri & 103 & 113 & 77 \\
\hline 3. Gaongo & 12 & 13 & 9 \\
\hline 4. Nafbanka & 28 & 31 & 21 \\
\hline 5. Gogo & 38 & 42 & 29 \\
\hline 6. Kopelin & 24 & 26 & 18 \\
\hline 7. Mouzi & 18 & 20 & 13 \\
\hline 8. Nagrigre & 27 & 30 & 20 \\
\hline 9. Samtinga & 17 & 19 & 13 \\
\hline 10.Tiougou & 25 & 27 & 19 \\
\hline 11. Yambasse & 16 & 19 & 12 \\
\hline TOTAL & 395 & 436 & 296 \\
\hline \multicolumn{4}{|l|}{$\begin{array}{l}\text { Région de l'EST : } \\
362 \text { ménages ; } \\
272 \text { adolescentes 10-19 ans }\end{array}$} \\
\hline 1. Fada & 181 & 199 & 136 \\
\hline 2. Bogandé & 65 & 72 & 49 \\
\hline 3. Mani & 33 & 36 & 24 \\
\hline 4. Koare & 22 & 24 & 16 \\
\hline 5. Natiaboani & 62 & 68 & 46 \\
\hline TOTAL & 363 & 399 & 271 \\
\hline \multicolumn{4}{|l|}{$\begin{array}{l}\text { Région du SAHEL : } \\
155 \text { ménages ; } \\
116 \text { adolescentes 10-19 ans }\end{array}$} \\
\hline 1.Dori & 90 & 99 & 67 \\
\hline 2. Gorom Gorom & 39 & 43 & 29 \\
\hline 3.Yakouta & 6 & 7 & 5 \\
\hline 4.Tibilédi & 8 & 9 & 6 \\
\hline 5. Saouga & 12 & 13 & 9 \\
\hline TOTAL & 155 & 171 & 116 \\
\hline \multicolumn{4}{|l|}{$\begin{array}{l}\text { Région du CENTRE-EST : } \\
482 \text { ménages ; } \\
362 \text { adolescentes 10-19 ans }\end{array}$} \\
\hline 1.Tenkodogo & 193 & 212 & 144 \\
\hline 2.Garango & 154 & 169 & 116 \\
\hline 3.Ouargaye & 43 & 47 & 33 \\
\hline 4.Moaga & $\mathbf{0}$ & $\mathbf{0}$ & $\mathbf{0}$ \\
\hline 5.Bissiga & 21 & 23 & 16 \\
\hline 6. Benna & 16 & 18 & 12 \\
\hline 7. Poestenga & 25 & 28 & 19 \\
\hline 8. Godin & 9 & 10 & 7 \\
\hline 9. Kinzeonguin & 11 & 12 & 8 \\
\hline 10.Koulbako & 9 & 10 & 7 \\
\hline TOTAL & 481 & 529 & 362 \\
\hline \multicolumn{4}{|l|}{$\begin{array}{l}\text { Région du CENTRE-NORD : } \\
344 \text { ménages ; } \\
258 \text { adolescentes } 10-19 \text { ans }\end{array}$} \\
\hline 1.Kaya & 234 & 257 & 175 \\
\hline 2.Boulsa & 76 & 84 & 57 \\
\hline 3.Pissila & 17 & 19 & 13 \\
\hline 4.Nessemtenga de Boussouma & 17 & 19 & 13 \\
\hline TOTAL & 344 & 379 & 258 \\
\hline TOTAL GENERAL & 1738 & 1914 & 1303 \\
\hline
\end{tabular}




\subsection{Les outils de collecte}

Deux questionnaires ont été utilisés pour la collecte des données : un questionnaire Ménage adressé au chef de ménage ou son répondant et un questionnaire individuel adressé aux adolescentes âgées de 10 à 19 ans. Un manuel d'instruction aux enquêteurs sur l'utilisation des questionnaires a été remis aux agents de terrain pour faciliter la collecte des données.

Le questionnaire Ménage comprend les modules suivants :

Module 0 : Identification du ménage

Module I : Caractéristiques sociodémographiques des membres du ménage

Module II : Possession de certains biens durables par le ménage :

Module III : Connaissances, attitudes, pratiques et perceptions du chef de ménage sur le mariage

Le questionnaire Individu adolescente comprend les modules suivants :

Module 0 : Identification

Module I : Caractéristiques de l'enquêtée

Module II : Instruction

Module III : Connaissances, attitudes, pratiques et perceptions du mariage

Module IV : Vécu du mariage chez les adolescentes mariées

Module V : Emploi et opportunités économiques

Module VI : Connaissances, attitudes et pratiques en matière de santé de la reproduction

Module VII : Mobilité des adolescentes

Module VIII : Travaux domestiques

Module IX : Relations et soutien communautaires.

\subsection{Recrutement, formation et sélection du personnel de terrain}

Le recrutement des enquêteurs à former s'est déroulé en deux étapes : dans un premier temps, il a été procédé à l'examen des Curriculum Vitae des 23 candidatures obtenues sur la base d'un certain nombre de critères (avoir au moins le niveau de la classe Terminale, avoir une expérience en matière d'enquête quantitative, maîtriser la langue parlée dans les localités et être immédiatement disponible). Dans un second temps, les candidats ont subi un entretien. A l’issue de cette présélection, 19 candidatures ont été retenues.

La formation sur les outils de collecte a duré trois jours (du 27 au 29 octobre 2008). Un prétest des outils a été effectué et les observations issues de celui-ci ont été intégrées dans les outils définitifs de collecte. A la fin de la formation, un test a été organisé en vue de retenir les enquêteurs (11) et superviseurs (4) qui ont conduit la collecte sur le terrain.

\subsection{Collecte, saisie et exploitation des données}

La collecte des données s'est déroulée du $1^{\mathrm{er}}$ au 22 novembre 2008 dans les 24 sites localisés dans 5 régions : le Centre-Nord, le Centre-Sud, le Centre-Est, l'Est et le Sahel. Quatre équipes dirigées chacune par un superviseur ont piloté l’opération.

Les consultants ont effectué des visites de supervision de cinq jours chacun auprès de l'équipe de terrain pour suivre et évaluer les progrès accomplis par les équipes et les aider à résoudre les problèmes rencontrés.

Les données ont été saisies avec EPIDATA pendant deux semaines.

L'exploitation des données a été faite avec SPSS. 


\subsection{Difficultés rencontrées}

Les difficultés relevées dans l’ensemble des sites se résument comme suit :

- la non disponibilité des enquêtés due à la période de récolte et des classes ce qui a obligé des enquêteurs à remettre les rendez-vous au soir voire dans la nuit;

- le volume du questionnaire jugé parfois trop long par bon nombre d'enquêtées entraînant ainsi une certaine lassitude chez les enquêtées ce qui obligeait les enquêteurs à se rendre plusieurs fois dans les ménages pour terminer l'entretien.

Cependant, ces difficultés n’ont pas empêchés de l'exécution du travail. 



\subsection{Caractéristiques sociodémographiques de la population étudiée}

\subsubsection{Caractéristiques sociodémographiques des membres du ménage}

L'âge, le sexe, le statut matrimonial, l'ethnie, la religion, le niveau d'instruction sont des caractéristiques sociodémographiques importantes dans l'analyse des phénomènes démographiques. Compte tenu de leur importance, un intérêt particulier a été accordé à leur enregistrement lors de l'enquête ménage.

\section{Structure par âge et par sexe de la population recensée}

Au total 1700 ménages ont été enquêtés. La population recensée lors de l'enquête ménage est de 10558 dont 4992 hommes et de 5566 femmes. Ces dernières représentent 52,7\% de l'effectif total. La population recensée est caractérisée par une forte proportion de jeunes. Les moins de 20 ans représentent 58\% de l'ensemble. Ces tendances s'observent dans toutes les cinq régions couvertes par l'étude.

Tableau 3.1 : Structure par âge et sexe de la population recensée

\begin{tabular}{lrrr}
\hline Groupe d'âges & \multicolumn{3}{c}{ Sexe } \\
\cline { 2 - 4 } & Masculin (\%) & Féminin (\%) & Ensemble (\%) \\
\hline $0-4$ ans & 15,2 & 12,0 & 13,5 \\
\hline $5-9$ ans & 14,6 & 13,7 & 14,1 \\
\hline $10-14$ ans & 13,6 & 15,6 & 14,7 \\
\hline $15-19$ ans & 13,1 & 18,0 & 15,7 \\
\hline $20-24$ ans & 8,6 & 8,6 & 8,6 \\
\hline $25-29$ ans & 7,2 & 7,0 & 7,1 \\
\hline $30-34$ ans & 6,2 & 5,6 & 5,9 \\
\hline $35-39$ ans & 5,1 & 5,1 & 5,1 \\
\hline $40-44$ ans & 3,9 & 4,1 & 3,0 \\
\hline $45-49$ ans & 3,0 & 3,0 & 2,6 \\
\hline $50-54$ ans & 3,0 & 2,3 & 1,5 \\
\hline $55-59$ ans & 1,6 & 1,4 & 1,5 \\
\hline $60-64$ ans & 1,8 & 1,3 & 1,0 \\
\hline $65-69$ ans & 1,1 & 0,9 & 0,8 \\
\hline $70-74$ ans & 1,0 & 0,7 & 0,4 \\
\hline $75-79$ ans & 0,6 & 0,3 & 0,2 \\
\hline $80-84$ ans & 0,2 & 0,2 & 0,2 \\
\hline 85 ans + & 0,1 & 0,3 & 100,0 \\
\hline Total & 100,0 & 100,0 & 10398 \\
\hline Effectif & 4906 & 5492 &
\end{tabular}

NB : 160 personnes (soit 1,6\% de la population) n’ont pas déclaré leur l’âge

\section{Statut matrimonial}

Les questions sur le statut matrimonial ont été renseignées pour toutes les femmes et pour tous les hommes de 10 ans ou plus des différents ménages sélectionnés.

Il ressort des analyses que plus de la moitié $(52,4 \%)$ de la population recensée est célibataire, $41,2 \%$ des individus sont mariés (28,7\% vivent en union monogamique et $12,5 \%$ en union polygamique). Les unions libres et les veufs/ves sont faiblement représentés avec 
respectivement 1,8\% et 3,1\% de l'ensemble. Les personnes fiancées ou promises de même que les divorcées/séparées représentent moins de 1\% de la population.

Tableau 3.2 : Répartition de la population selon le statut matrimonial et la région

\begin{tabular}{lrrrrrr}
\hline Etat matrimonial & \multicolumn{7}{c}{ Région } \\
\cline { 2 - 7 } & Centre-Sud & Est & Sahel & Centre-Est & Centre-Nord & Ensemble \\
\hline Célibataire & 50,2 & 53,3 & 51,5 & 56,0 & 50,3 & 52,4 \\
\hline Marié monogame & 28,6 & 24,3 & 34,7 & 28,7 & 30,2 & 28,7 \\
\hline Marié(e) polygame & 16,7 & 12,3 & 6,4 & 10,4 & 13,6 & 12,5 \\
\hline Union libre & 0,4 & 4,7 & 0,2 & 1,5 & 1,7 & 1,8 \\
\hline Divorcé(e)/séparé(e) & 0,6 & 0,9 & 2,4 & 0,8 & 0,7 & 0,9 \\
\hline Veuf (ve) & 2,8 & 3,7 & 4,1 & 2,5 & 3,0 & 3,1 \\
\hline Promise & 0,0 & 0,1 & 0,1 & 0,0 & 0,2 & 0,1 \\
\hline Fiancée & 0,7 & 0,5 & 0,5 & 0,1 & 0,3 & 0,4 \\
\hline Total & 100,0 & 100,0 & 100,0 & 100,0 & 100,0 & 100,0 \\
\hline Effectif & 1867 & 1688 & 824 & 2049 & 1743 & 8171 \\
\hline
\end{tabular}

Par ailleurs, le statut matrimonial varie selon le sexe. Ainsi, les femmes sont plus en union polygamique (15,4\% contre $9,2 \%)$, les hommes plus célibataires ( $56,8 \%$ contre $48,7 \%)$ et plus mariés monogames (30,8\% contre 27\%). Le pourcentage de veuves et de divorcées/séparées est plus important chez les femmes que chez les hommes.

Graphique 3.1 : Répartition de la population recensée selon le statut matrimonial et le sexe (en \%)

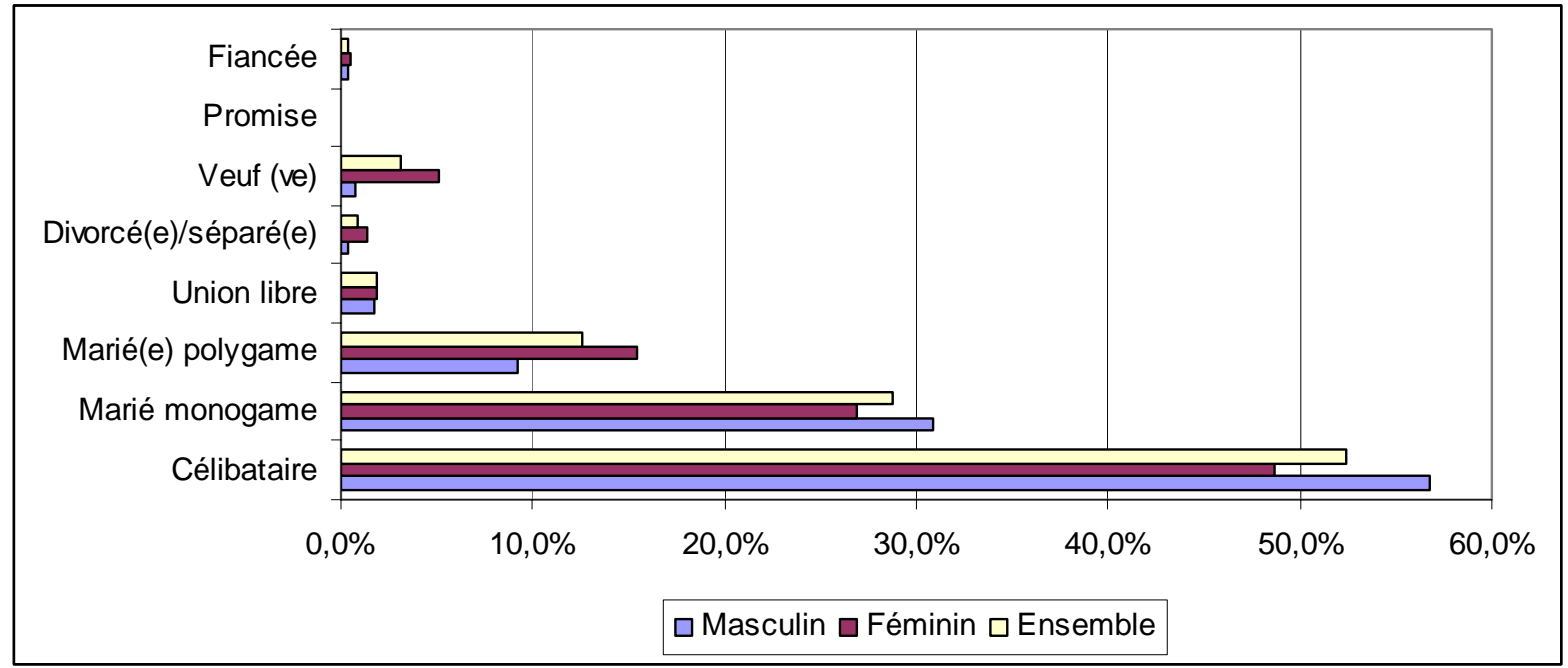

Effectif $=8171$

\subsubsection{Caractéristiques sociodémographiques des chefs de ménage}

\section{Sexe des chefs de ménage}

Sur l'ensemble des ménages enquêtés (1700), les hommes chefs de ménages représentent $89,41 \%$ alors que les femmes chefs de ménage ne représentent que 10,59\%. Cette tendance s'observe au niveau des différentes régions. En effet, les pourcentages d'hommes chefs de ménage varient entre $86,7 \%$ (région de l'Est) et 92,5\% (région du Centre-Sud) tandis que celles de femmes chefs de ménage varient entre 7,5\% (région du Centre-Sud) et 13,3\% (région de l'Est). 
Les adolescentes chefs de ménage représentent 9,2\% des femmes chefs de ménage, soit moins de $1 \%$ de l'ensemble des chefs de ménage.

Tableau 3.3 : Répartition par sexe des chefs de ménages enquêtés selon la région (\%)

\begin{tabular}{lrrrrrr}
\hline Sexe & \multicolumn{5}{c}{ Région } \\
\cline { 2 - 7 } & Centre-Sud & Est & Sahel & Centre-Est & Centre-Nord & Ensemble \\
\hline Masculin & 92,48 & 86,65 & 90,58 & 89,96 & 87,93 & 89,41 \\
\hline Féminin & 7,52 & 13,35 & 9,42 & 10,04 & 12,07 & 10,59 \\
\hline Total & 100,00 & 100,00 & 100,00 & 100,00 & 348 & 100,00 \\
\hline Effectif & 359 & 367 & 138 & 488 & 1700 \\
\hline
\end{tabular}

\section{Instruction et alphabétisation}

La répartition des chefs de ménage selon la fréquentation scolaire passée montre qu'une forte proportion (52,6\%) de ceux-ci n'est pas allée à l'école. La majorité des chefs de ménage n’ont pas fréquenté l'école. Cette forte proportion des chefs de ménage qui n’ont pas été à l'école s'observe également pour la région du Centre-Sud (62,7\%), la région du Sahel (55,8\%) et la région de l'Est $(53,4 \%)$. Dans le Centre-Nord, la proportion des chefs de ménage ayant été à l'école est juste la moitié (50,6\%) alors que dans le Centre-Est, cette proportion est supérieure (54\%) à celle des chefs de ménage n’ayant pas été à l’école.

Graphique 3.2 : Répartition de chefs de ménage selon la fréquentation scolaire passée (\%)

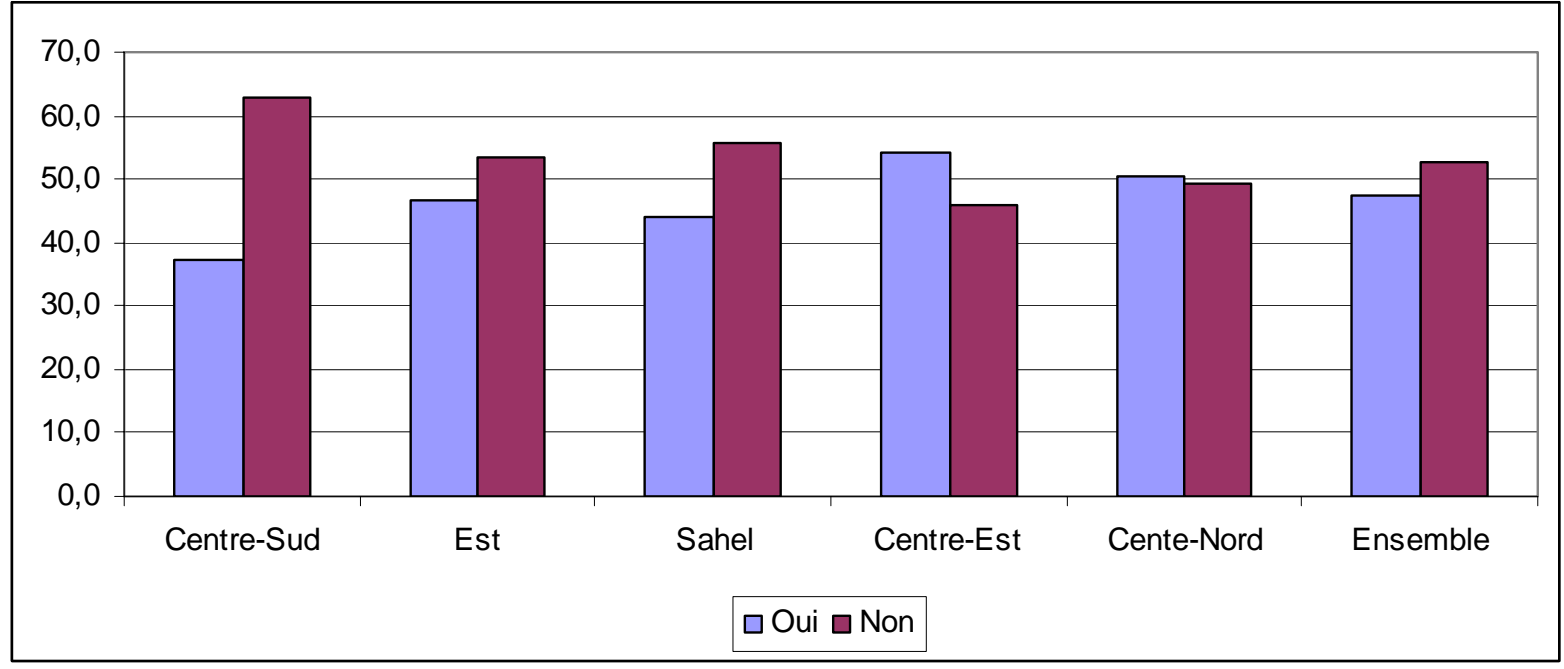

Effectifs : Centre-Sud (359), Est (367), Sahel (138), Centre-Est (488), Centre-Nord (348), Ensemble (1700)

Sur un ensemble de 1700 chefs de ménage enquêtés, seulement 796 (soit 47\%) ont été à l'école. Parmi ces derniers, $45,4 \%$ ont atteint le niveau primaire et $44,6 \%$ le niveau secondaire. Ceux ayant atteint le niveau supérieur ne représentent que 8,7\% des chefs de ménage instruits.

Dans les régions du Sahel et du Centre-Est, les chefs de ménage ayant un niveau d'instruction primaire sont les plus nombreux : 54,7\% dans le Centre-Est et 55,7\% dans le Sahel. Dans le Centre-Sud, l'Est et le Centre-Nord, la majorité des chefs de ménage ont atteint un niveau d'instruction secondaire. 
Concernant le niveau d'instruction supérieur, le Sahel suivi de la région de l'Est, enregistrent les faibles proportions des chefs de ménage ayant atteint ce niveau. Ces proportions sont de 3,3\% et 5,4\% respectivement pour le Sahel et pour l'Est. Dans le Centre-Sud, le Centre-Est et le Centre-Nord, les proportions de chefs de ménage ayant le niveau d'instruction supérieur sont respectivement de $11,3 \% ; 10,1 \%$ et $9,7 \%$.

Pour ce qui concerne les femmes chefs de ménage, il ressort que sur un total de 180, 74 d'entre elles (soit 41,3\%) ont été à l'école. Parmi celles-ci, 31,5\% se sont limitées au niveau primaire, $63 \%$ au niveau secondaire et seulement $5,5 \%$ au niveau supérieur.

Tableau 3.4 : Répartition des chefs de ménage par niveau d’instruction selon la région (\%)

\begin{tabular}{lrrrrrr}
\hline \multirow{2}{*}{$\begin{array}{l}\text { Niveau } \\
\text { d'instruction }\end{array}$} & Centre-Sud & Est & Sahel & Centre-Est & Centre-Nord & Ensemble \\
\cline { 2 - 8 } & 36,8 & 41,1 & 55,7 & 54,7 & 38,6 & 45,4 \\
\hline Primaire & 48,9 & 53,6 & 39,3 & 34,9 & 48,9 & 44,6 \\
\hline Secondaire & 11,3 & 5,4 & 3,3 & 10,1 & 9,7 & 8,7 \\
\hline Supérieur & 3 & 0 & 1,6 & 0,4 & 2,8 & 1,4 \\
\hline Ne sait pas & 100 & 100 & 100 & 100 & 100 & 100 \\
\hline Total & 134 & 171 & 61 & 264 & 176 & 806 \\
\hline Effectif & & & & & &
\end{tabular}

Selon l'alphabétisation, le constat qui ressort est qu'une forte proportion (63,2\%) des chefs de ménage ne savent pas lire ni écrire dans une langue quelconque. C'est dans la région de l'Est qu'on enregistre la plus faible proportion des chefs de ménage sachant lire et écrire dans une langue quelconque; en effet, seulement un peu plus d'un chef de ménage sur cinq est alphabétisé. C'est dans les régions du Sahel et du Centre-Nord qu'on observe les plus fortes proportions des chefs de ménages qui sont alphabétisés. Les proportions sont de $46 \%$ et de $45,6 \%$.

Graphique 3.3 : Répartition des chefs de ménage par région selon qu’ils soient alphabétisés ou non (\%)

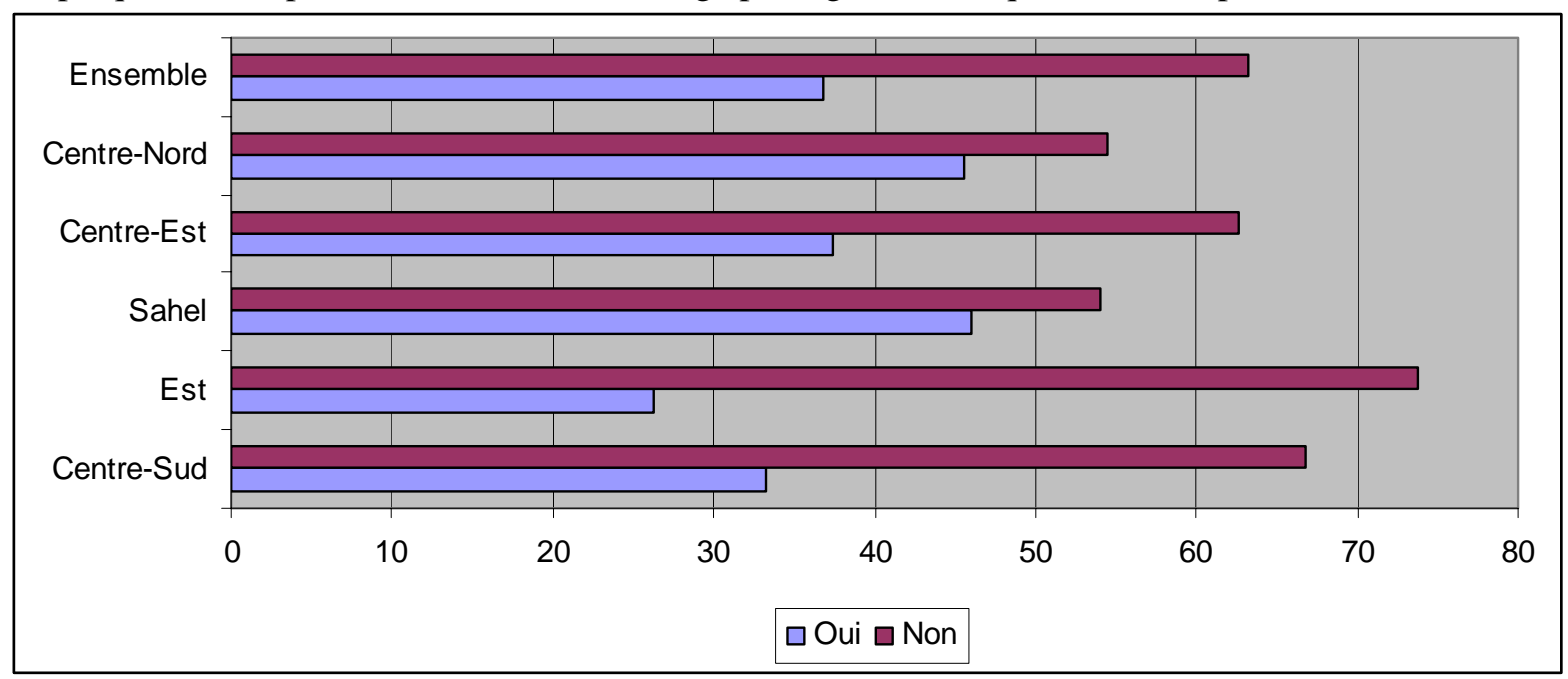

Effectifs : Centre-Sud (225), Est (196), Sahel (77), Centre-Est (224), Centre-Nord (172), Ensemble (894) 


\section{Milieu de résidence}

La répartition des chefs de ménage selon le milieu de résidence montre qu'environ $70 \%$ de ceux-ci vivent en milieu urbain contre $30 \%$ qui vivent en milieu rural.

Graphique 3.4: Répartition des chefs de ménages selon le milieu de résidence

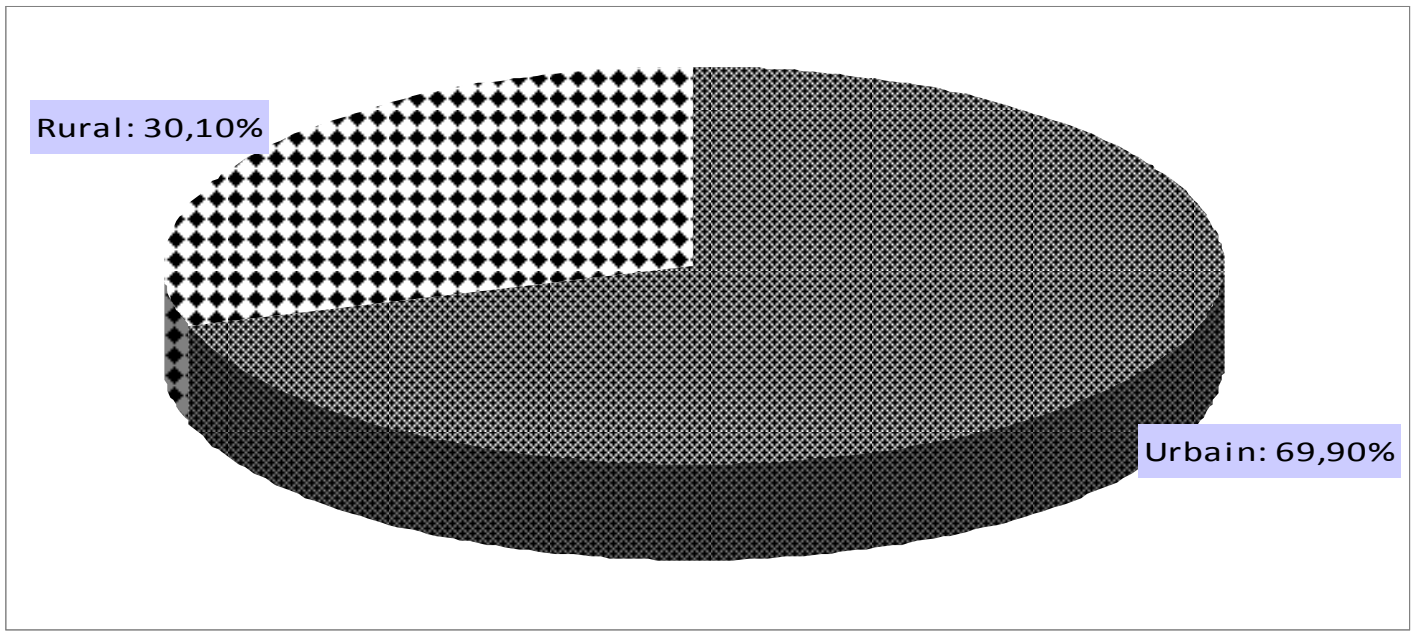

\section{Occupation principale des chefs de ménage}

La répartition des chefs de ménage selon leur occupation principale montre que la majorité $(43,7 \%)$ de ceux-ci exerce dans l'agriculture. Ce constat s'observe aussi pour toutes les régions. Dans la région du Centre-Sud, la proportion des chefs de ménage occupés par l'agriculture est très élevée soit six chefs de ménage sur dix. Le commerce est aussi une activité exercée par une proportion non négligeable $(13,5 \%)$ de chefs de ménage. cette activité est exercée par 22,2\% de chefs de ménage dans le Centre-Est. Les autres catégories d'activités (artisanat, élevage, ménagère, élève/étudiants) sont faiblement représentées chez les chefs de ménage.

Tableau 3.5 : Répartition par région des chefs de ménage selon l’occupation principale (en \%)

\begin{tabular}{lrrrrrr}
\hline $\begin{array}{l}\text { Occupation } \\
\text { principale }\end{array}$ & Centre-Sud & Est & Sahel & Centre-Est & Centre-Nord & Ensemble \\
\cline { 2 - 8 } Aucun & 1,1 & 1,1 & 0 & 1 & 5,8 & 2 \\
\hline Agriculture & 60,2 & 44,1 & 31,6 & 39,9 & 36 & 43,7 \\
\hline Elevage & 2,8 & 0,8 & 0,7 & 1,4 & 0 & 1,2 \\
\hline Artisanat & 0 & 1,9 & 4,4 & 3,3 & 0,9 & 1,9 \\
\hline Commerce & 8,6 & 9 & 14 & 22,2 & 11 & 13,5 \\
\hline Ménagère & 1,4 & 5,2 & 0,7 & 2,3 & 4,7 & 3,1 \\
\hline Elevé & 1,1 & 5,7 & 2,2 & 1,9 & 0,9 & 2,3 \\
\hline Autre & 24,8 & 32,2 & 46,3 & 27,8 & 40,1 & 32,1 \\
\hline Total & 100 & 100 & 100 & 100 & 100 & 100 \\
\hline Effectif & 359 & 367 & 138 & 488 & 348 & 1700 \\
\hline
\end{tabular}

\subsubsection{Caractéristiques sociodémographiques des adolescentes}

Au total, 1320 adolescentes ont été enquêtées dans les cinq régions selon la répartition suivante : 295 (22,3\%) dans le Centre-Sud, 288 (21,82\%) à l’Est, 116 (8,8\%) au Sahel, 361 (27,3\%) du Centre-Est, et 260 (19,7\%) dans le Centre-Nord. 


\section{Age des adolescentes enquêtées}

L'enquête s'est intéressée aux adolescentes de 10 à 19 ans. Dans l'ensemble, la moyenne d'âges de celles-ci se situe à 15 ans et les adolescentes de 19 ans sont les plus nombreuses. Excepté la région du Sahel où 50\% des adolescentes ont moins de 17 ans. Dans les quatre autres régions, la moitié des adolescentes enquêtées a moins de 15 ans (50\%).

S'agissant de leurs conjoints, on note que plus de 32\% des adolescentes mariées n'ont pas réussi à donner l'âge de leur conjoint. La moyenne d'âges des conjoints est de 24 ans dans l'ensemble et varie entre 22,6 ans dans le Sahel et 27,6 ans dans le Centre-Est.

Tableau 3.6 : Répartition des adolescentes par âge (en \%)

\begin{tabular}{lrrrrrr}
\hline Age de & \multicolumn{7}{c}{ Région } \\
\cline { 2 - 8 } l'individu & Centre-Sud & Est & Sahel & Centre-Est & Centre-Nord & Ensemble \\
\hline 10 & 5,1 & 4,9 & 0,9 & 10,9 & 8,9 & 7,0 \\
\hline 11 & 5,5 & 2,4 & 1,8 & 6,4 & 5,8 & 4,8 \\
\hline 12 & 10,2 & 7,7 & 7,1 & 11,7 & 9,7 & 9,7 \\
\hline 13 & 8,2 & 11,5 & 9,7 & 12,3 & 8,1 & 10,2 \\
\hline 14 & 9,2 & 12,9 & 8,0 & 7,8 & 9,3 & 9,5 \\
\hline 15 & 12,6 & 13,6 & 9,7 & 15,9 & 10,0 & 13,0 \\
\hline 16 & 13,0 & 12,2 & 12,4 & 7,5 & 12,0 & 11,1 \\
\hline 17 & 10,2 & 10,5 & 23,0 & 10,3 & 9,7 & 11,3 \\
\hline 18 & 11,3 & 11,9 & 14,2 & 5,6 & 10,4 & 9,9 \\
\hline 19 & 14,7 & 12,2 & 13,3 & 11,7 & 16,2 & 13,5 \\
\hline Total & 100,0 & 100,0 & 100,0 & 100,0 & 100,0 & 100,0 \\
\hline Effectif & 293 & 286 & 113 & 359 & 259 & 1310 \\
\hline Mode & 19,0 & 15,0 & 17,0 & 15,0 & 19,0 & 19,0 \\
\hline Médiane & 15,0 & 15,0 & 17,0 & 15,0 & 15,0 & 15,0 \\
\hline Moyenne & 15,2 & 15,2 & 15,9 & 14,4 & 15,0 & 15,0 \\
\hline Ecart type & 2,7 & 2,5 & 2,3 & 2,8 & 2,9 & 2,7 \\
\hline
\end{tabular}

NB : 6 adolescentes n’ont pas déclaré leur âge et 4 ne savent pas leur âge.

\section{Ethnie des adolescentes}

Sur l'ensemble des adolescentes enquêtées, 60,7\% sont des Mossi, 12,9\% de l'ethnie gourmantché, $10,8 \%$ de bissa et $7,6 \%$ de peulh.

Les adolescentes dans les régions du Centre-Sud et du Centre-Nord sont majoritairement des Mossi qui représentent respectivement 91,9\% et 91,2\% de l'ensemble. Le Centre-Est aussi englobe plus d'adolescentes Mossi (52,1\%) suivies par les Bissa qui constituent 36,5\% de l'ensemble des adolescentes). Au Sahel, les adolescentes Peulh sont plus représentées (59,3\%) et à l’Est, on a les Gourmantché (56,3\%) suivies par les Mossi (33\%).

- $\quad 2$ Le mode est la valeur de la variable statistique dont la fréquence est la plus élevée.

- La médiane est la valeur de la variable statistique qui partage en deux effectifs égaux, la série des observations préalablement ordonnées par valeur croissante ou décroissante du caractère : 50\% des observations ont une valeur inférieure ou égale à la médiane, et 50\% des valeurs ont une valeur supérieure ou égale à la médiane.

- La moyenne algébrique est la valeur que prendrait la variable statistique si la masse totale était uniformément répartie entre les unités statistiques de la population.

- L'écart type mesure la dispersion des valeurs de la variable statistique autour de leur moyenne. 
Tableau 3.7 : Répartition des adolescentes enquêtées selon l’ethnie (en \%)

\begin{tabular}{lrrrrrr}
\hline \multirow{2}{*}{ Ethnie } & \multicolumn{7}{c}{ Région } \\
\cline { 2 - 8 } & Centre-Sud & Est & Sahel & Centre-Est & Centre-Nord & Ensemble \\
\hline Mossi & 91,9 & 33,0 & 7,1 & 52,1 & 91,2 & 60,7 \\
\hline Peulh & 1,7 & 2,8 & 59,3 & 3,6 & 2,7 & 7,6 \\
\hline Gourmantché & 0,3 & 56,3 & 2,7 & 0,8 & 0,0 & 12,9 \\
\hline Bissa & 0,3 & 1,7 & 2,7 & 36,5 & 0,8 & 10,8 \\
\hline Dioula & 0,3 & 0,3 & 0,0 & 0,8 & 0,0 & 0,4 \\
\hline Autre & 5,4 & 5,9 & 28,3 & 6,1 & 5,4 & 7,7 \\
\hline Total & 100,0 & 100,0 & 100,0 & 100,0 & 100,0 & 100,0 \\
\hline Effectif & 295 & 288 & 116 & 361 & 260 & 1320 \\
\hline
\end{tabular}

\section{Religion}

Parmi les adolescentes enquêtées, plus de la moitié (54,4\%) sont des musulmanes, 36,1\% des catholiques, $8,4 \%$ sont des protestantes et seulement $1 \%$ d'animistes.

L'appartenance religieuse des adolescentes est du même ordre que celle des chefs de ménage. Les adolescentes du Centre-Sud sont principalement de religion catholique $(52,2 \%)$ ou musulmane $(41,3 \%)$.

Celles de l'Est sont principalement de religion musulmane (40,6\%), ou catholique (33\%) et protestante (25,3\%). Au Sahel, elles sont à 97,3\% de religion musulmane alors qu'au CentreEst elles sont à 58,1\% de religion musulmane et à 37,7\% de religion catholique. Au CentreNord, elles sont à 60,8\% de religion musulmane et à 33,5\% de religion catholique.

Tableau 3.8 : Répartition des adolescentes selon la religion (en \%)

\begin{tabular}{lrrrrrr}
\hline Religion & \multicolumn{7}{c}{ Région } & & \\
& Centre-Sud & Est & Sahel & Centre-Est & Centre-Nord & Ensemble \\
\hline Musulman & 41,3 & 40,6 & 97,3 & 58,1 & 60,8 & 54,4 \\
\hline Catholique & 52,2 & 33,0 & 2,7 & 37,7 & 33,5 & 36,1 \\
\hline Protestant & 5,8 & 25,3 & 0,0 & 3,6 & 2,7 & 8,4 \\
\hline Animiste & 0,7 & 1,0 & 0,0 & 0,6 & 3,1 & 1,1 \\
\hline Total & 100,0 & 100,0 & 100,0 & 100,0 & 100,0 & 100,0 \\
\hline Effectif & 295 & 288 & 116 & 361 & 260 & 1320 \\
\hline
\end{tabular}

\section{Situation matrimoniale}

La grande majorité des adolescentes enquêtées sont célibataires (86,2\%), les mariées représentent $9,3 \%$ (7,6\% de monogames et $1,7 \%$ de polygames). Celles qui sont en union libre représentent $1,3 \%$ alors que les promises $(0,5 \%)$, les fiancées $(2 \%)$ et les divorcées/séparées $(0,7 \%)$ sont très peu nombreuses. Cette tendance s’observe dans les différentes régions. 
Tableau 3.9 : Situation matrimoniale des adolescentes (en \%)

\begin{tabular}{lrrrrrr}
\hline Situation matrimoniale & \multicolumn{7}{c}{ Région } \\
\cline { 2 - 8 } & Centre-Sud & Est & Sahel & Centre-Est & Centre-Nord & Ensemble \\
\hline Célibataire & 83,7 & 89,6 & 72,6 & 88,9 & 87,7 & 86,2 \\
\hline Mariée (ménage monogame) & 7,5 & 5,6 & 22,1 & 5,0 & 7,3 & 7,6 \\
\hline Mariée (ménage polygame) & 2,4 & 2,4 & 0,0 & 0,8 & 1,9 & 1,7 \\
\hline Union libre & 1,0 & 0,3 & 0,0 & 2,8 & 1,2 & 1,3 \\
\hline Promise & 0,3 & 0,7 & 0,9 & 0,3 & 0,8 & 0,5 \\
\hline Fiancée & 4,4 & 1,4 & 2,7 & 0,8 & 1,2 & 2,0 \\
\hline Divorcée/séparée & 0,7 & 0,0 & 1,8 & 1,4 & 0,0 & 0,7 \\
\hline Total & 100,0 & 100,0 & 100,0 & 100,0 & 100,0 & 100,0 \\
\hline Effectif & 295 & 288 & 116 & 361 & 260 & 1320 \\
\hline
\end{tabular}

\section{Niveau d'instruction}

Sur les 1320 adolescentes enquêtées, 969 (soit 73,4\%) ont été à l'école. Parmi celles-ci, plus de la moitié $(51,4 \%)$ ont atteint le niveau primaire et $48,1 \%$ le niveau secondaire. Tandis que dans le Centre-Sud et le Centre-Est, la plupart des adolescentes ayant fréquenté ont atteint le niveau primaire, la tendance est inversée à l'Est, au Sahel et au Centre-Nord où c'est le niveau secondaire qui prédomine.

Chez les conjoints des adolescentes mariées, seulement $29 \%$ et $16 \%$ ont respectivement le niveau primaire et secondaire.

Tableau 3.10 : Niveau d'instruction des adolescentes (en \%)

\begin{tabular}{lrrrrrr}
\hline Haut niveau d'enseignement atteint & \multicolumn{7}{c}{ Région } \\
\cline { 2 - 7 } & Centre-Sud & Est & Sahel & Centre-Est & Centre-Nord & Ensemble \\
\hline Primaire & 60,8 & 32,2 & 39,4 & 67,0 & 44,3 & 51,4 \\
\hline Secondaire & 38,6 & 67,8 & 60,6 & 32,7 & 54,7 & 48,1 \\
\hline Medersa & 0,5 & 0,0 & 0,0 & 0,0 & 0,5 & 0,2 \\
\hline Ne sait pas & 0,0 & 0,0 & 0,0 & 0,3 & 0,5 & 0,2 \\
\hline Total & 100,0 & 100,0 & 100,0 & 100,0 & 100,0 & 100,0 \\
\hline Effectif & 189 & 211 & 71 & 295 & 203 & 969 \\
\hline
\end{tabular}

Dans toutes les régions, une forte proportion des adolescentes sont toujours à l'école, les proportions vont de 70,4\% au Centre-Sud à 85,7\% au Centre-Nord.

Graphique 3.5: Fréquentation scolaire actuelle (en \%)

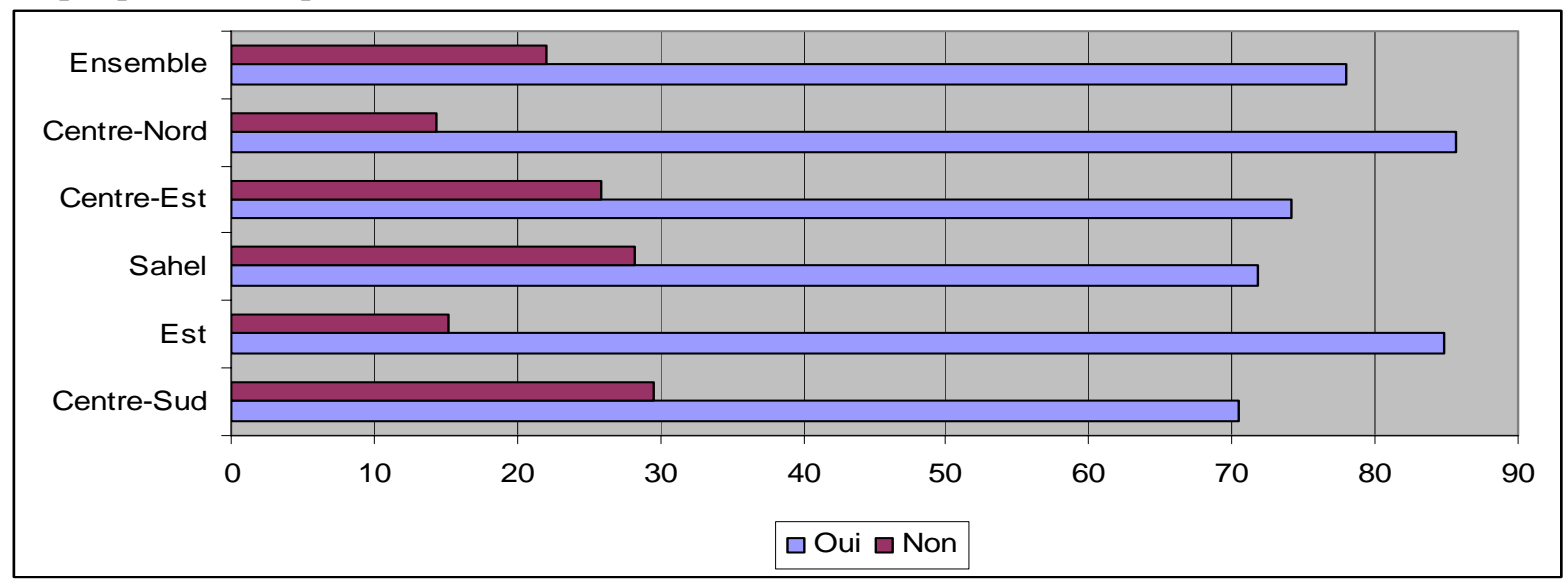

Effectifs : Centre-Sud (189), Est (211), Sahel (71), Centre-Est (295), Centre-Nord (203), Ensemble (989) 


\section{Milieu de résidence}

Sur l'ensemble des adolescentes enquêtées, la majorité vit en milieu urbain3 (70,2\%) contre $29,8 \%$ en milieu rural.

Graphique 3.6 : Répartition des adolescentes enquêtées selon le milieu de résidence (en \%)

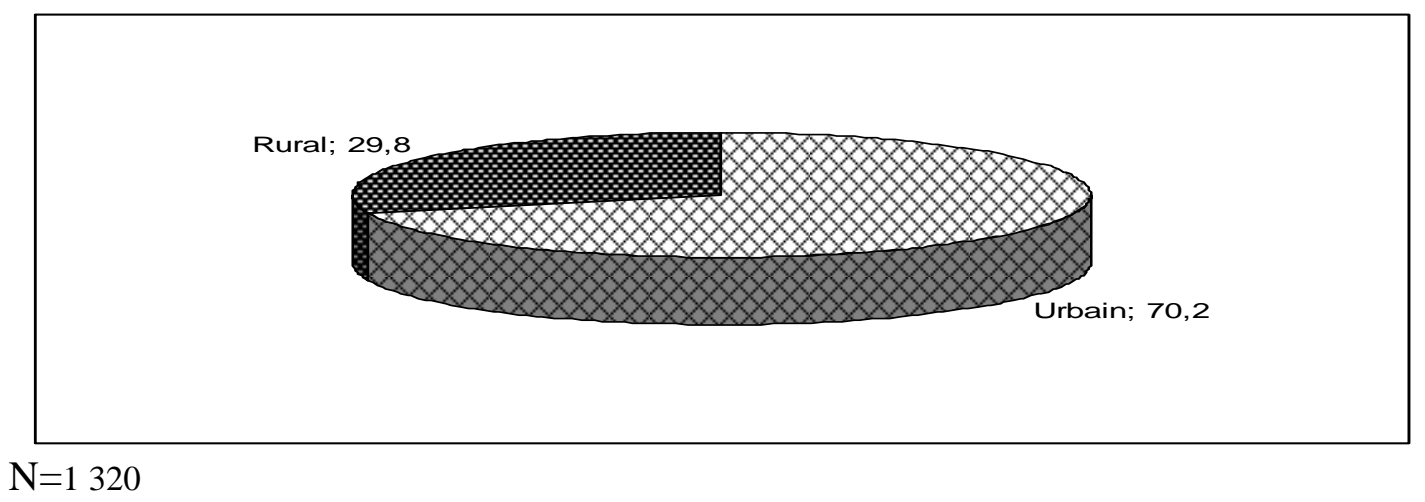

\subsection{Connaissances des textes et de certaines dispositions légales concernant les adolescentes}

\subsubsection{Chez les chefs de ménages}

\section{Mariage}

Le Code des Personnes et de la Famille du Burkina Faso (Burkina Faso, 1991) stipule en son article 238 que «le mariage ne peut être contracté qu'entre un homme âgé de plus de vingt ans et une femme de plus de dix-sept ans, sauf dispense d'âge accordée pour motif grave par le tribunal civil. Cette dispense d'âge ne peut être accordée en aucun cas pour un homme ayant moins de dix-huit ans et une femme ayant moins de quinze ans ». Il apparaît au vu des résultats, que cette information est peu connue par les enquêtés.

Dans l'ensemble, 46,1\% des chefs de ménage connaissent que la législation prévoit un âge au mariage pour les filles et pour les garçons contre 14,3\% qui disent qu'il n'existe pas de législation en la matière et 39,6\% qui déclarent ne rien savoir.

Selon les régions, c'est au Centre-Est que plus de la moitié des chefs de ménage (52,5\%) ne savent pas que la législation prévoit un âge minimum pour le mariage des filles et des garçons.

Cependant parmi ceux qui ont déclaré savoir que la législation prévoit un âge minimum au mariage des filles et des garçons, très peu d'entre eux connaissent cet âge. En effet, 25\% des personnes ont donné la bonne réponse sur l'âge minimum au mariage des filles (contre 37,1\% qui connaissent l'âge minimum au mariage des garçons).

\footnotetext{
${ }^{3}$ Le milieu urbain correspond aux chefs lieu des différentes provinces concernées par l'enquête.
} 
Tableau 3.11 : Connaissance des chefs de ménage de l'âge minimum légal au mariage (en \%)

\begin{tabular}{|c|c|c|c|c|c|c|}
\hline \multirow{2}{*}{$\begin{array}{l}\text { Connaissez-vous que la législation } \\
\text { prévoit un âge au mariage pour les } \\
\text { filles/garcons }\end{array}$} & \multicolumn{6}{|c|}{ Région } \\
\hline & Centre-Sud & Est & Sahel & Centre-Est & Centre-Nord & Ensemble \\
\hline Oui & 47,6 & 47,1 & 63,0 & 33,6 & 54,5 & 46,1 \\
\hline Non & 15,1 & 12,0 & 5,1 & 13,9 & 20,1 & 14,3 \\
\hline Ne sait pas & 37,3 & 40,9 & 31,9 & 52,5 & 25,4 & $\overline{39,6}$ \\
\hline Total & 100,0 & 100,0 & 100,0 & 100,0 & 100,0 & $\overline{100,0}$ \\
\hline Effectif & 359 & 367 & 138 & 488 & 348 & 1700 \\
\hline \multicolumn{7}{|l|}{ Age légal au mariage de filles } \\
\hline 14 & 1,2 & 0,0 & 0,0 & 0,0 & 0,0 & 0,3 \\
\hline 15 & 1,8 & 1,1 & 4,5 & 1,8 & 0,5 & 1,6 \\
\hline 16 & 0,0 & 1,1 & 0,0 & 12,6 & 2,1 & $\overline{3,4}$ \\
\hline 17 & 27,1 & 21,8 & 13,6 & 21,0 & 38,1 & 25,9 \\
\hline 18 & 28,8 & 42,0 & 46,6 & 32,9 & 32,0 & 35,3 \\
\hline 19 & 12,9 & 7,5 & 2,3 & 3,0 & 5,7 & 6,7 \\
\hline 20 & 16,5 & 16,7 & 27,3 & 15,0 & 11,3 & 16,1 \\
\hline 21 & 5,9 & 5,7 & 5,7 & 5,4 & 4,1 & $\overline{5,3}$ \\
\hline Ne sait pas & 5,9 & 4,0 & 0,0 & 8,4 & 6,2 & $\overline{5,4}$ \\
\hline Total & 100,0 & 100,0 & 100,0 & 100,0 & 100,0 & $\overline{100,0}$ \\
\hline Effectif & 171 & 173 & 87 & 164 & 190 & 784 \\
\hline \multicolumn{7}{|l|}{ Age légal des garçons au mariage } \\
\hline 17 & 0,6 & 0,0 & 0,0 & 1,2 & 1,5 & 0,8 \\
\hline 18 & 4,7 & 4,0 & 1,1 & 3,0 & 6,2 & 4,2 \\
\hline 19 & 1,8 & 0,0 & 0,0 & 1,8 & 6,2 & 2,3 \\
\hline 20 & 34,3 & 29,9 & 42,0 & 53,9 & 29,4 & 37,1 \\
\hline 21 & 13,6 & 1,7 & 0,0 & 1,8 & 8,2 & 5,7 \\
\hline 22 & 3,0 & 11,5 & 9,1 & 4,2 & 5,7 & 6,4 \\
\hline 23 & 3,0 & 1,7 & 2,3 & 0,0 & 0,5 & 1,4 \\
\hline 24 & 0,6 & 2,9 & 0,0 & 1,2 & 0,0 & 1,0 \\
\hline 25 & 15,4 & 19,0 & 33,0 & 19,8 & 22,2 & 20,7 \\
\hline 26 & 9,5 & 12,1 & 9,1 & 4,8 & 10,3 & $\overline{9,2}$ \\
\hline Ne sait pas & 13,6 & 17,2 & 3,4 & 8,4 & 9,8 & 11,2 \\
\hline Total & 100,0 & 100,0 & 100,0 & 100,0 & 100,0 & 100,0 \\
\hline Effectif & 170 & 174 & 88 & 167 & 194 & 793 \\
\hline
\end{tabular}

\section{Scolarisation}

Droit élémentaire reconnu par la convention relative aux droits de l'enfant (signée le 20 novembre 1989) que le Burkina a faite sienne, l'éducation figure au rang des secteurs prioritaires pour le développement du pays. Dans cette optique, la «Loi d'orientation de l'Education » adoptée en 1996 au Burkina Faso, dans le but d'atteindre la scolarisation universelle, a énoncé le principe de scolarité obligatoire de 6 à 16 ans. Il faut néanmoins souligner que l'âge minimum de scolarisation obligatoire était jusqu'à ces dernières années de 7 ans.

Selon le DECRET No 2008-236/ PRES/PM/MEBA/MESSRS/MASSN/MATD du 08 mai 2008 portant organisation de l'enseignement primaire, l'âge minimum de scolarisation obligatoire au Burkina a été de 6 ans. 
Contrairement au mariage, une majorité des chefs de ménage connaissent que la législation prévoit un âge de scolarisation obligatoire des enfants (98,6\%). 64,7\% de ceux-ci ont déclaré que cet âge est de 7 ans contre 30,2\% qui ont cité l'âge de 6 ans.

Tableau 3.12 : Connaissance de l’âge de scolarisation obligatoire selon la législation

\begin{tabular}{lrrrrrr}
\hline \multirow{2}{*}{$\begin{array}{l}\text { Connaissance de l'âge de scolarisation } \\
\text { Oui }\end{array}$} & \multicolumn{6}{c}{ Région } \\
\cline { 2 - 7 } & Centre-Sud & Est & Sahel & Centre-Est & Centre-Nord & Ensemble \\
\hline Non & 99,4 & 98,6 & 100,0 & 98,4 & 97,7 & 98,6 \\
\hline Ne sait pas & 0,0 & 0,8 & 0,0 & 1,0 & 0,6 & 0,6 \\
\hline Total & 0,6 & 0,5 & 0,0 & 0,6 & 1,7 & 0,8 \\
\hline Effectif & 100,0 & 100,0 & 100,0 & 100,0 & 100,0 & 100,0 \\
\hline & 359 & 367 & 138 & 488 & 348 & 1700 \\
\hline Age légal à la scolarisation obligatoire & & & & & & \\
\hline 4 & & & & & & \\
\hline 5 & 1,4 & 1,4 & 0,0 & 0,2 & 0,6 & 0,8 \\
\hline 6 & 2,8 & 5,0 & 0,7 & 1,7 & 2,9 & 2,8 \\
\hline 7 & 39,4 & 37,3 & 30,7 & 16,9 & 31,9 & 30,2 \\
\hline 8 & 56,1 & 55,2 & 65,7 & 78,3 & 64,0 & 64,7 \\
\hline 9 & 0,0 & 0,6 & 1,5 & 2,1 & 0,3 & 0,9 \\
\hline 10 ans ou plus & 0,0 & 0,0 & 0,7 & 0,0 & 0,0 & 0,1 \\
\hline Ne sait pas & 0,3 & 0,3 & 0,7 & 0,6 & 0,3 & 0,4 \\
\hline Total & 0,0 & 0,3 & 0,0 & 0,2 & 0,0 & 0,1 \\
\hline Effectif & 100,0 & 100,0 & 100,0 & 100,0 & 100,0 & 100,0 \\
\hline & 359 & 367 & 138 & 488 & 348 & 1700 \\
\hline
\end{tabular}

\section{Emploi}

Le travail des enfants qui fait l'objet d'une attention soutenue de la part de l'opinion publique internationale depuis un certain nombre d'années est réglementé au plan international et national. Ainsi, l’âge minimum légal d'exercice d'un emploi est fixé à 15 ans au plan international selon les conventions de l'OIT. Au niveau national, le Code Social, en ses articles 15 et 87 du Code du Travail de 1992, stipule que "Nul ne peut être engagé avant l'âge de 14 ans, même comme apprenti...l'âge minimum d'admission à tout type d'emploi ou de travail ne devra pas être inférieur à quatorze (14) ans ». Le Code du Travail a été revu en 2008 et par rapport à l'âge d'entrée sur le marché du travail, l’âge de 16 ans est désormais en vigueur (art. 152).

Concernant l'emploi, 66,6\% des chefs de ménage déclarent savoir que la législation prévoit un âge minimum légal pour un travail payé contre $19 \%$ d'entre eux qui ne le savent pas et $14,4 \%$ qui déclarent qu'il n’y a aucune disposition en la matière.

Cependant, très peu $(2,5 \%)$ de chefs de ménage connaissent que cet âge est fixé à 16 ans contre un peu plus du quart (26,3\%) qui fixent l’âge légal à la majorité à 18 ans. 
Tableau 3.13 : Connaissance des chefs de ménage de l’âge minimum légal pour un travail payé (en \%)

\begin{tabular}{lrrrrrr}
\hline $\begin{array}{l}\text { Connaissance de l'âge minimum } \\
\text { légal pour un travail payé }\end{array}$ & \multicolumn{7}{c}{ Région } \\
\cline { 2 - 8 } & Centre-Sud & \multicolumn{1}{c}{ Est } & \multicolumn{1}{c}{ Sahel } & Centre-Est & Centre-Nord & Ensemble \\
\hline Oui & 74,8 & 71,9 & 68,8 & 52,7 & 71,3 & 66,6 \\
\hline Non & 8,7 & 12,3 & 2,2 & 24,3 & 13,6 & 14,4 \\
\hline Ne sait pas & 16,5 & 15,8 & 29,0 & 23,0 & 15,1 & 19,0 \\
\hline Total & 100,0 & 100,0 & 100,0 & 100,0 & 100,0 & 100,0 \\
\hline Effectif & $\mathbf{3 5 9}$ & $\mathbf{3 6 7}$ & $\mathbf{1 3 8}$ & $\mathbf{4 8 8}$ & $\mathbf{3 4 8}$ & $\mathbf{1 7 0 0}$ \\
\hline & & & & & & \\
\hline Age légal au travail & & & & & & \\
\hline 14 & 12,4 & 3,0 & 27,4 & 40,8 & 20,3 & 19,7 \\
\hline 15 & 15,7 & 8,0 & 14,7 & 10,8 & 14,2 & 12,4 \\
\hline 16 & 4,9 & 2,3 & 1,1 & 0,4 & 2,8 & 2,5 \\
\hline 17 & 14,6 & 4,6 & 5,3 & 1,2 & 9,3 & 7,3 \\
\hline 18 & 21,0 & 33,8 & 21,1 & 23,1 & 29,3 & 26,3 \\
\hline 19 & 4,9 & 3,4 & 1,1 & 1,5 & 3,3 & 3,1 \\
\hline 20 & 15,7 & 26,2 & 25,3 & 10,4 & 13,0 & 17,2 \\
\hline 21 ans ou plus & 10,5 & 14,4 & 3,2 & 8,5 & 7,3 & 9,6 \\
\hline Ne sait pas & 0,4 & 4,2 & 1,1 & 3,5 & 0,4 & 2,0 \\
\hline Total & 100,0 & 100,0 & 100,0 & 100,0 & 100,0 & 100,0 \\
\hline Effectif & $\mathbf{2 6 9}$ & $\mathbf{2 6 4}$ & $\mathbf{9 5}$ & $\mathbf{2 5 7}$ & $\mathbf{2 4 8}$ & $\mathbf{1 1 3 2}$ \\
\hline
\end{tabular}

\subsubsection{Chez les adolescentes}

\section{Connaissances sur le Code des Personnes et de la Famille}

Excepté le Sahel où 34,8\% des adolescentes déclarent avoir entendu parler du Code des Personnes et de la Famille, d'une manière générale, les adolescentes sont sous-informées. Parmi celles qui en ont entendu parler, 65,8\% déclarent savoir que le code prévoit un âge minimum pour le mariage des filles et des garçons. Selon les régions, ce pourcentage varie de $42,9 \%$ à l'Est, à $80 \%$ au Sahel.

Une infime proportion d'adolescentes qui se situe entre $1,1 \%$ et $12,5 \%$, a participé à une séance de sensibilisation sur le Code des Personnes et de la Famille et dans la plupart des cas, c'est l'Action Sociale qui a fait cette sensibilisation.

\section{Age minimum pour le mariage de la fille et du garçon que prévoit le Code selon les déclarations des adolescentes}

L’âge minimum pour le mariage de la fille, déclaré par les adolescentes sur la base de leur connaissance du Code, est de 18 ans dans l'ensemble de la zone d'intervention. Il varie entre 16 et 20 ans, respectivement au Centre-Est et au Sahel. Le pourcentage de réponses correctes est de $24,6 \%$ pour les cinq régions. Ce pourcentage varie de $23,1 \%$ au Centre-Sud à $37,5 \%$ au Centre-Nord.

Cet âge est de 20 ans pour les garçons dans l'ensemble des cinq régions et aussi dans le Centre-Sud, l'Est et le Centre-Est. Il est de 19 et 25 ans respectivement dans le Centre-Nord et le Sahel. Le pourcentage de réponses correctes est de 36,2\%. Il varie de $25 \%$ au Centre-Nord à $100 \%$ à l’Est. 
Tableau 3.14 : Age minimum pour le mariage de la fille et du garçon que prévoit le Code selon les déclarations des adolescentes

\begin{tabular}{|c|c|c|c|c|c|c|}
\hline \multirow{2}{*}{$\begin{array}{l}\text { Age minimum pour le mariage } \\
\text { de la fille et du garçon (en } \\
\text { années) d'après le Code selon la } \\
\text { déclaration des adolescentes }\end{array}$} & \multicolumn{6}{|c|}{ Région } \\
\hline & Centre-Sud & Est & Sahel & Centre-Est & Centre-Nord & Ensemble \\
\hline $\begin{array}{l}\text { Age minimum pour la fille } \\
\text { (Mode) }\end{array}$ & 18 & $15,17,18,19$ & 20 & 16 & 17,18 & 18 \\
\hline Pourcentage & 61,5 & $25 \%$ chacun & 40 & 35,7 & $\begin{array}{l}37,5 \% \\
\text { chacun }\end{array}$ & 27,5 \\
\hline $\begin{array}{l}\text { Pourcentage de réponses correctes } \\
\text { (17 ans) }\end{array}$ & 23,1 & 25 & 23,3 & 21,4 & 37,5 & 24,6 \\
\hline $\begin{array}{l}\text { Age minimum pour le garçon } \\
\text { (Mode) }\end{array}$ & 20 & 20 & 25 & 20 & 19,25 & 20 \\
\hline Pourcentage & 46,2 & 100 & 33,3 & 64,3 & $25 \%$ chacun & 36,2 \\
\hline $\begin{array}{l}\text { Pourcentage de réponses correctes } \\
\text { (20 ans) }\end{array}$ & 46,2 & 100 & 13,3 & 64,3 & 25 & 36,2 \\
\hline Effectif & 13 & 4 & 30 & 14 & 8 & 69 \\
\hline
\end{tabular}

N.B. : 8,8\% d'adolescentes n’ont pas pu donner un âge minimum parmi 78.

Le contenu du Code des Personnes et de la Famille est très peu connu par les adolescentes. Excepté au Sahel où près de $60 \%$ des adolescentes déclarent que le mariage civil est celui reconnu par la loi, moins de $50 \%$ des adolescentes dans les autres régions ont pu le reconnaître.

Tableau 3.15 : Type de mariage reconnu par la loi

\begin{tabular}{lrrrrrr}
\hline Type de mariage reconnu par la loi & \multicolumn{7}{c}{ Région } \\
\cline { 2 - 8 } & \multicolumn{1}{c}{ Centre-Sud } & \multicolumn{1}{c}{ Est } & Sahel & Centre-Est & Centre-Nord & Ensemble \\
\hline Mariage civil & 30,8 & 37,1 & 59,8 & 35,5 & 46,5 & 39,1 \\
\hline Mariage religieux & 15,1 & 17,1 & 3,6 & 3,4 & 7,8 & 9,9 \\
\hline Mariage coutumier & 3,4 & 3,1 & 0,0 & 0,0 & 2,7 & 2,0 \\
\hline Union libre & 0,0 & 0,0 & 0,0 & 0,0 & 0,4 & 0,1 \\
\hline Ne sait pas & 50,7 & 42,7 & 36,6 & 61,2 & 42,6 & 49,0 \\
\hline Total & 100,0 & 100,0 & 100,0 & 100,0 & 100,0 & 100,0 \\
\hline Effectif & $\mathbf{2 9 5}$ & $\mathbf{2 8 8}$ & $\mathbf{1 1 6}$ & $\mathbf{3 6 1}$ & $\mathbf{2 6 0}$ & $\mathbf{1 3 2 0}$ \\
\hline
\end{tabular}

\section{Eléments constitutifs d'un dossier de mariage}

En général dans les 5 régions, les éléments constitutifs d'un dossier de mariage sont très peu connus par les adolescentes.

Moins de 50\% d'entre elles en général savent que l'acte de naissance ou le jugement supplétif fait partie du dossier de mariage.

Moins de 30\% savent que le certificat de résidence fait partie du dossier de mariage.

Moins de 21\% savent que le certificat de visite prénuptiale en fait partie.

Environ 21\% savent que le contrat de mariage s'il y a lieu fait partie du dossier de mariage.

Environ 16\% des adolescentes savent que la déclaration d'option de polygamie fait partie du dossier de mariage si tel est le choix des futurs époux.

Moins de 40\% d'entre elles savent que la photocopie certifiée conforme de la carte d'identité des témoins fait partie et moins de $15 \%$ des adolescentes savent que le certificat de nongrossesse pour une femme veuve ou divorcée en fait partie. 
Tableau 3.16 : Connaissance des éléments constitutifs d'un dossier de mariage

\begin{tabular}{|c|c|c|c|c|c|c|}
\hline \multirow{2}{*}{$\begin{array}{l}\text { Connaissance des éléments constitutifs d'un } \\
\text { dossier de mariage }\end{array}$} & \multicolumn{6}{|c|}{ Région } \\
\hline & Centre-Sud & Est & Sahel & Centre-Est & Centre-Nord & Total \\
\hline Acte de naissance/jugement supplétif de chacun & 33,8 & 53,1 & 53,6 & 36,5 & 45,2 & 42,7 \\
\hline Certificat de résidence pour chacun & 21,8 & 39,9 & 25,0 & 19,2 & 29,0 & 26,7 \\
\hline Certificat de visite prénuptiale & 15,1 & 31,1 & 9,8 & 15,9 & 25,9 & 20,5 \\
\hline Un contrat de mariage & 18,1 & 22,4 & 6,3 & 18,4 & 30,1 & 20,5 \\
\hline Consentement du père et de la mère/tuteur & 29,4 & 45,5 & 34,8 & 31,2 & 38,2 & 35,6 \\
\hline La déclaration d'option de polygamie & 14,3 & 14,3 & 15,2 & 18,4 & 15,1 & 15,7 \\
\hline Photocopie certifiée des CIB des témoins & 25,6 & 46,5 & 45,5 & 33,2 & 32,8 & $\overline{35,4}$ \\
\hline Une autorisation administrative & 11,9 & 36,0 & 7,1 & 24,2 & 20,1 & 21,8 \\
\hline $\begin{array}{l}\text { Un certificat de non-grossesse pour femme } \\
\text { veuve/divorcée }\end{array}$ & 10,2 & 16,1 & 8,9 & 17,3 & 15,1 & 14,3 \\
\hline Effectif & 295 & 288 & 116 & 361 & 260 & 1320 \\
\hline
\end{tabular}

Par ailleurs, la grande majorité des adolescentes enquêtées (72\%) ne savent pas par quel document officiel on peut reconnaître que le mariage a été célébré. Seulement, 25\% des adolescentes citent le certificat de mariage comme document officiel d'acte de mariage.

Tableau 3.17 : Connaissance du document officiel de mariage (en \%)

\begin{tabular}{lrrrrrr}
\hline Document officiel reconnaissant le mariage & \multicolumn{7}{c}{ Région } \\
\cline { 2 - 7 } & Centre-Sud & Est & Sahel & Centre-Est & Centre-Nord & Ensemble \\
\hline Certificat de mariage & 20,5 & 16,8 & 42,0 & 23,7 & 34,0 & 25,1 \\
\hline Contrat de mariage & 1,7 & 1,7 & 0,0 & 0,0 & 1,5 & 1,1 \\
\hline Autre & 3,1 & 1,0 & 0,9 & 0,0 & 4,6 & 1,9 \\
\hline Ne sait pas & 74,7 & 80,4 & 57,1 & 76,3 & 59,8 & 72,0 \\
\hline Total & 100,0 & 100,0 & 100,0 & 100,0 & 100,0 & 100,0 \\
\hline Effectif & 295 & 288 & 116 & 361 & 260 & 1320 \\
\hline
\end{tabular}

\section{Conditions à remplir pour se marier légalement au Burkina Faso}

Il ressort que d'une manière générale, les conditions pour se marier légalement au Burkina Faso ne sont pas bien maîtrisées par les adolescentes.

Dans l'ensemble, $11,8 \%$ des adolescentes déclarent «que les deux conjoints doivent être obligatoirement de sexe différent », 11,6\% citent l'âge obligatoire selon le Code, 22,6\% citent le consentement des 2 conjoints. 
Tableau 3.18 : Connaissance des conditions à remplir pour se marier légalement au Burkina Faso (en $\%)$

\begin{tabular}{lrrrrrr}
\hline $\begin{array}{l}\text { Conditions à remplir pour se marier } \\
\text { légalement au Burkina Faso }\end{array}$ & \multicolumn{7}{c}{ Région } \\
\cline { 2 - 8 } & Centre-Sud & Est & Sahel & Centre-Est & Centre-Nord & Ensemble \\
\hline -Etre obligatoirement de sexe différent & 12,3 & 3,8 & 5,4 & 19,5 & 12,4 & $11,8^{4}$ \\
\hline -Avoir au moins 17 ans pour une fille et 20 & 6,5 & 17,8 & 6,3 & 8,4 & 17,4 & 11,6 \\
pour un garçon & & & & & & \\
\hline -Dérogation du juge & 0,7 & 0,0 & 0,0 & 0,3 & 1,2 & 0,5 \\
\hline -Par consentement mutuel des futurs époux & 6,5 & 7,7 & 30,4 & 4,5 & 18,5 & 10,6 \\
\hline -Consentement des 2 conjoints & 20,5 & 15,4 & 36,6 & 29,2 & 17,8 & 22,6 \\
\hline -Consentement des parents & 14,3 & 29,0 & 44,6 & 30,4 & 14,3 & 24,5 \\
\hline -Autres & 6,1 & 2,1 & 2,7 & 0,6 & 5,4 & 3,3 \\
\hline -NSP & 60,1 & 57,0 & 27,7 & 58,5 & 54,4 & 55,1 \\
\hline Effectif & 295 & 288 & 116 & 361 & 260 & 1320 \\
\hline
\end{tabular}

\section{Connaissances des conditions pour s'opposer à un mariage}

D’une manière générale, les conditions d'opposition au mariage sont mal connues par les adolescentes. Environ 30\% citent comme conditions l'absence de consentement des 2 conjoints, l'absence de consentement des parents.

Tableau 3.19 : Connaissance des conditions pour s’opposer à un mariage (en \%)

\begin{tabular}{|c|c|c|c|c|c|c|}
\hline \multirow{2}{*}{$\begin{array}{l}\text { Dans quel cas, peut-on s'opposer à un } \\
\text { mariage? }\end{array}$} & \multicolumn{6}{|c|}{ Région } \\
\hline & Centre-Sud & Est & Sahel & Centre-Est & Centre-Nord & Ensemble \\
\hline $\begin{array}{l}\text { Si votre conjoint vous a caché sa véritable } \\
\text { identité }\end{array}$ & 6,5 & 7,7 & 0,0 & 11,7 & 8,1 & 8,0 \\
\hline Impuissance /stérilité Maladie mentale & 13,3 & 3,8 & 0,0 & 4,7 & 12,0 & 7,5 \\
\hline Maladie mentale & 6,1 & 3,1 & 1,8 & 7,2 & 5,4 & 5,3 \\
\hline Passé pénal & 3,1 & 0,7 & 0,0 & 1,9 & 4,6 & 2,3 \\
\hline S'il avait déjà une femme non déclarée & 9,9 & 1,7 & 0,0 & 5,3 & 8,1 & 5,7 \\
\hline $\begin{array}{l}\text { Si votre conjoint était légalement marié } \\
\text { (régime monogame) }\end{array}$ & 3,4 & 4,2 & 0,9 & 2,5 & 3,9 & 3,2 \\
\hline $\begin{array}{l}\text { Si vous ou votre conjoint n'a pas l'âge } \\
\text { requis }\end{array}$ & 1,0 & 16,4 & 9,0 & 7,5 & 5,0 & 7,6 \\
\hline Sans le consentement des 2 conjoints & 12,3 & 22,0 & 84,7 & 43,5 & 18,5 & 30,4 \\
\hline Sans le consentement des parents & 12,3 & 45,1 & 64,9 & 57,1 & 13,9 & 36,6 \\
\hline Autre & 49,3 & 33,2 & 2,7 & 20,3 & 51,7 & 34,4 \\
\hline Effectif & 295 & 288 & 116 & 361 & 260 & 1320 \\
\hline
\end{tabular}

Environ 50\% des adolescentes déclarent connaître des structures officielles auxquelles elles peuvent recourir en cas de conflits conjugaux, mais lorsqu'il s'agit de les citer, c'est surtout l'Action Sociale qui revient fréquemment. Les autres adolescentes ne connaissent aucune structure et affirment se référer souvent aux parents pour régler ces conflits.

\footnotetext{
${ }^{4}$ Le fait d'être obligatoirement de sexe différent est probablement évident pour la plupart des adolescentes, ce qui pourrait expliquer le faible pourcentage de déclaration pour cette condition.
} 
Tableau 3.20. Les structures de recours en cas de conflits conjugaux (en \%)

\begin{tabular}{|c|c|c|c|c|c|c|}
\hline \multirow{2}{*}{$\begin{array}{l}\text { En cas d'un conflit entre conjoints dans } \\
\text { un foyer, à qui ou à quelles structures } \\
\text { peuvent-ils recourir? }\end{array}$} & \multicolumn{6}{|c|}{ Région } \\
\hline & Centre-Sud & Est & Sahel & Centre-Est & Centre-Nord & Ensemble \\
\hline Tribunal/Justice & 1,4 & 6,3 & 8 & 5,1 & $\overline{2,3}$ & 4,2 \\
\hline Police & 3,1 & 7 & 3,6 & 6 & 3,5 & 4,8 \\
\hline Action sociale & 9,9 & 23,8 & 6,3 & 10,3 & 15,1 & 13,8 \\
\hline Autorités coutumières/religieuses & 19,5 & 18,2 & 8,9 & 25,1 & 15,1 & 18,9 \\
\hline Parents & 70,5 & 58 & 92 & 82,1 & 64,9 & 71,6 \\
\hline Témoins de mariage & 18,8 & 6,6 & 14,3 & 22,5 & 17,8 & 16,5 \\
\hline Autorités administratives et communales & 5,5 & 22 & 10,7 & 10,8 & 5 & 10,9 \\
\hline Autre & 4,1 & 4,2 & 9,8 & 1,4 & 5 & 4,1 \\
\hline NSP & 15,1 & 10,5 & 1,8 & 6,9 & 24,7 & 12,6 \\
\hline Effectif & 112 & 127 & 99 & 232 & 90 & 657 \\
\hline \multicolumn{7}{|l|}{$\begin{array}{l}\begin{array}{l}\text { Savez-vous qu'il existe des structures } \\
\text { officielles qui vous protègent }\end{array} \\
\end{array}$} \\
\hline Non & 62,2 & 55,9 & 14,7 & 35,6 & 65,2 & 50,2 \\
\hline Oui & 37,8 & 44,1 & 85,3 & 64,4 & 34,8 & 49,8 \\
\hline Total & 100 & 100 & 100 & 100 & 100 & 100 \\
\hline Effectif & 112 & 127 & 99 & 232 & 90 & 657 \\
\hline \multicolumn{7}{|l|}{$\begin{array}{l}\text { Structures officielles qui peuvent } \\
\text { protéger les adolescentes en cas de conflit } \\
\text { entre époux }\end{array}$} \\
\hline Tribunal/Justice & 12,6 & 17,6 & 54,6 & 17,6 & 7,7 & 20,9 \\
\hline Police & 14,4 & 12,8 & 15,5 & 33 & 16,5 & 21 \\
\hline Action sociale & 61,3 & 60 & 20,6 & 43,2 & 62,6 & 48,8 \\
\hline Autorités coutumières/religieuses & 4,5 & 16,8 & 25,8 & 28,6 & 5,5 & 18,6 \\
\hline Parents & 13,5 & 8 & 35,1 & 37,4 & 15,4 & 24,3 \\
\hline Témoins de mariage & 0,9 & 3,2 & 6,2 & 8,4 & 1,1 & 4,8 \\
\hline Autorités administratives et communales & 9 & 28 & 33 & 8,8 & 8,8 & 16,1 \\
\hline Autre & 4,5 & 0,8 & 0 & 0 & 7,7 & 2 \\
\hline NSP & 4,5 & 4,1 & 2,1 & 0,4 & 5 & 3 \\
\hline Effectif & 112 & 127 & 99 & 232 & 90 & 657 \\
\hline
\end{tabular}

\subsection{Perceptions sur le mariage des adolescents/tes}

\subsubsection{Chez les chefs de ménage}

\section{Age au mariage des filles dans les localités}

Le mariage des adolescentes est précoce dans les différentes régions concernées par l'enquête. En effet, plus de la moitié $(55,2 \%)$ des chefs de ménage déclarent que les adolescentes se marient avant 18 ans dans leurs localités.

Le Sahel se distingue des autres régions pour ce qui concerne la précocité du mariage des jeunes filles. Dans cette région, plus de $15 \%$ des chefs de ménage affirment que les filles se marient entre 10 et 14 ans et 47,8\% donnent la fourchette d'âge de 15 à 17 ans. Selon donc les propos des chefs de ménage environ $63,7 \%$ des filles au sahel se marient avant leur dixhuitième anniversaire. 
Tableau 3.21 : Age au mariage chez les filles selon la perception des chefs de ménage (en \%)

\begin{tabular}{lrrrrrr}
\hline $\begin{array}{l}\text { Groupe d'âges de mariage } \\
\text { des filles dans la localité }\end{array}$ & \multicolumn{7}{c}{ Région } \\
\cline { 2 - 8 } $10-14$ ans & Centre-Sud & Est & Sahel & Centre-Est & Centre-Nord & Ensemble \\
\hline $15-17$ ans & 2,0 & 1,9 & 15,9 & 2,9 & 0,9 & 3,1 \\
\hline 18 ans ou plus & 52,1 & 41,4 & 47,8 & 56,9 & 58,4 & 52,1 \\
\hline Ne sait pas & 37,5 & 50,7 & 33,3 & 34,7 & 36,9 & 39,1 \\
\hline Total & 8,4 & 6,0 & 2,9 & 5,5 & 3,8 & 5,7 \\
\hline Effectif & 100,0 & 100,0 & 100,0 & 100,0 & 100,0 & 100,0 \\
\hline & 359 & 367 & 138 & 488 & 348 & 1700 \\
\hline
\end{tabular}

Par rapport aux filles, les garçons se marient plus tardivement. En général, dans les différentes localités les garçons se marient entre 20 et 29 ans selon les chefs de ménage.

Tableau 3.22 : Age au mariage chez les garçons selon la perception des chefs de ménage (en \%)

\begin{tabular}{lrrrrrr}
\hline Groupe d'âges de mariage & \multicolumn{7}{c}{ Région } \\
\cline { 2 - 8 } des garçons dans la localité & Centre-Sud & Est & Sahel & Centre-Est & Centre-Nord & Ensemble \\
\hline Moins de 15 ans & 0,3 & 0,3 & 0,0 & 0,4 & 0,3 & 0,3 \\
\hline $15-19$ ans & 11,8 & 9,5 & 7,2 & 11,5 & 9,3 & 10,3 \\
\hline $20-24$ ans & 44,0 & 47,7 & 43,5 & 65,1 & 40,6 & 50,1 \\
\hline $25-29$ ans & 28,6 & 26,7 & 32,6 & 14,6 & 33,3 & 25,4 \\
\hline $30-34$ ans & 2,5 & 3,8 & 3,6 & 1,4 & 5,2 & 3,1 \\
\hline 35 ans ou plus & 0,3 & 0,3 & 0,0 & 0,0 & 0,6 & 0,2 \\
\hline Ne sait pas & 12,6 & 11,7 & 13,0 & 7,0 & 10,7 & 10,4 \\
\hline Total & 100,0 & 100,0 & 100,0 & 100,0 & 100,0 & 100,0 \\
\hline Effectif & 359 & 367 & 138 & 488 & 348 & 1700 \\
\hline
\end{tabular}

\section{Perception et Pratique sur la dot chez les chefs de ménage}

La dot est l'ensemble des cadeaux que l'un des mariés offre à sa nouvelle famille. Cette une vieille tradition africaine encore appliquée aujourd'hui. Dans la plupart des cas, la dot est offerte par l'homme à la famille de la femme. Les parents de la jeune fille exigent aussi la dot pour s'assurer qu'ils ont laissé leur fille en de bonnes mains.

Elle est essentiellement composée de présents pour la famille : pagnes pour les femmes, alcool pour les hommes (le plus souvent, il s'agit d'alcools traditionnels). Il peut également s’agir de mets destinés à la mère (viande, légumes, condiments, épices, huiles végétales,...) et qui traduit la gratitude du futur marié pour avoir élevé sa fiancée jusqu'à maturité (car en général, l'éducation de la jeune fille est le fruit des efforts maternels).

Au départ symbolique dans biens de sociétés au Burkina Faso, la dot est en train de perdre cet esprit pour devenir commercial.

L'article 244 du Code des Personnes et de la Famille stipule que «le versement de la dot soit en espèces soit en nature, soit sous forme de prestation de service est illégal » au Burkina Faso.

Cependant, cette pratique est encore courante dans les différentes localités couvertes par l'enquête. En effet, 90\% des chefs de ménages ont déclaré que la dot est encore pratiquée dans leur localité. La pratique de la dot reste obligatoire dans les localités selon $66,7 \%$ des chefs de ménage. Cette proportion varie de 99,3\% au Sahel à 87\% à l’Est. 
A la question de savoir ce que les chefs de ménage pensent de la pratique de la dot, 79,4\% pensent que cette pratique est une bonne chose contre $7,6 \%$ et $8,7 \%$ qui trouvent qu'elle est respectivement une mauvaise chose et une pratique dépassée.

Tableau 3.23 : Perception de la dot par les chefs de ménage (en \%)

\begin{tabular}{|c|c|c|c|c|c|c|}
\hline \multirow[t]{2}{*}{ Pratique de la dot } & \multicolumn{6}{|c|}{ Région } \\
\hline & Centre-Sud & Est & Sahel & Centre-Est & Centre-Nord & Ensemble \\
\hline Oui & 88,6 & 87 & 99,3 & 92,2 & 87,8 & 90 \\
\hline Non & 11,4 & 13 & 0,7 & 7,8 & 12,2 & 10 \\
\hline Total & 100 & 100 & 100 & 100 & 100 & 100 \\
\hline Effectif & 359 & 367 & 138 & 488 & 348 & 1700 \\
\hline \multicolumn{7}{|c|}{ Pratique de la dot est-elle obligatoire? } \\
\hline Oui & 63 & 55,6 & 97,8 & 67,3 & 68,6 & 66,7 \\
\hline Non & 37 & 44,4 & 2,2 & 32,7 & 31,4 & 33,3 \\
\hline Total & 100 & 100 & 100 & 100 & 100 & 100 \\
\hline Effectif & 359 & 367 & 138 & 488 & 348 & 1700 \\
\hline \multicolumn{7}{|l|}{ Opinion sur la dot } \\
\hline Bonne chose & 79,6 & 73,5 & 91,4 & 81,5 & 77,7 & 79,4 \\
\hline Mauvaise chose & 6,5 & 13 & 4,3 & 4,5 & 8,4 & 7,6 \\
\hline Pratique dépassée & 4,2 & 10,5 & 4,3 & 13,2 & 7 & 8,7 \\
\hline Autre & 9,6 & 3 & & 0,8 & 7 & 4,3 \\
\hline Total & 100 & 100 & 100 & 100 & 100 & 100 \\
\hline Effectif & 359 & 367 & 138 & 488 & 348 & 1700 \\
\hline
\end{tabular}

\section{Perceptions générales}

En plus des questions directement liées aux connaissances relatives au mariage, nous avons recueilli les opinions des chefs de ménage sur l'importance de la scolarisation des filles d'une part et sur la possibilité qu'une femme exerce une activité économique d'autre part.

La quasi-totalité $(98,8 \%)$ des chefs de ménage pense que c'est une bonne chose de scolariser les filles. Les raisons évoquées sont diverses : 61,6\% pensent que c'est un droit humain de scolariser les filles. Les pourcentages de déclaration des autres raisons tels que le pouvoir de se prendre en charge, le meilleur encadrement des enfants, la contribution aux dépenses du ménage et pour une meilleure réceptivité des messages de sensibilisation, tournent autour de $30 \%$.

Pour ce qui est de l'exercice d'une activité économique par la femme, 92,3\% des chefs de ménage pensent qu'elle peut le faire librement. 
Tableau 3.24 : Opinions des chefs de ménage sur la scolarisation des filles et l'exercice d'une activité économique par la femme (en \%)

\begin{tabular}{lrrrrrr}
\hline Opinion sur la scolarisation des filles & \multicolumn{7}{c}{ Région } \\
\cline { 2 - 7 } & Centre-Sud & Est & Sahel & Centre-Est & Centre-Nord & Ensemble \\
\hline Oui & 99,1 & 99,2 & 99,3 & 97,5 & 99,4 & 98,8 \\
\hline Non & 0,9 & 0,8 & 0,7 & 2,5 & 0,6 & 1,2 \\
\hline Total & 100,0 & 100,0 & 100,0 & 100,0 & 100,0 & 100,0 \\
\hline Effectif & 359 & 367 & 138 & 488 & 348 & 1700 \\
\hline
\end{tabular}

\begin{tabular}{lrrrrrrr}
\hline $\begin{array}{l}\text { Raisons pour la scolarisation des } \\
\text { filles }\end{array}$ & & & & & & & \\
\hline C'est droit humain & 30,6 & 16,1 & 6,5 & 42,9 & 32,7 & 29,3 \\
\hline Peut se prendre en charge & 48,9 & 61,3 & 85,5 & 71,8 & 51,3 & 61,6 \\
\hline Peut mieux encadrer ses enfants & 21,4 & 13,9 & 20,3 & 43,6 & 31,2 & 28,0 \\
\hline $\begin{array}{l}\text { Peut contribuer aux dépenses du } \\
\text { ménage }\end{array}$ & 23,7 & 29,7 & 56,5 & 37,5 & 30,0 & 32,9 \\
\hline $\begin{array}{l}\text { Peut mieux comprendre et réagir aux } \\
\text { messages de sensibilisation. }\end{array}$ & 41,1 & 19,9 & 97,1 & 12,4 & 32,7 & 31,2 \\
\hline Autre & 31,8 & 44,1 & 6,5 & 20,8 & 22,5 & 27,4 \\
\hline Effectif & 359 & 367 & 138 & 488 & 348 & 1700 \\
\hline
\end{tabular}

La femme peut-elle exercer librement une activité économique ?

\begin{tabular}{lrrrrrr}
\hline Oui & 96,6 & 79,2 & 93,5 & 99,2 & 91,6 & 92,3 \\
\hline Non & 3,4 & 20,8 & 6,5 &, 8 & 8,4 & 7,7 \\
\hline Total & 100,0 & 100,0 & 100,0 & 100,0 & 100,0 & 100,0 \\
\hline Effectif & 359 & 367 & 138 & 488 & 348 & 1700 \\
\hline
\end{tabular}

\subsubsection{Chez les adolescentes}

\section{Perceptions des adolescentes sur l'âge auquel les filles et les garçons se marient généralement ou peuvent se marier dans les régions}

Selon les déclarations des adolescentes, l'âge auquel les filles se marient généralement est de 17 ans pour l'ensemble des régions. Le Centre-Sud et le Centre-Nord présentent cette tendance alors que le Sahel, le Centre-Est et l'Est affichent respectivement 15, 16 et 18 ans. Quant aux garçons, les déclarations des adolescentes font ressortir qu'ils se marient généralement à 20 ans, quelle que soit la région.

S'agissant de l'âge à partir duquel une fille peut se marier, le consensus tourne autour de 20 ans, quelle que soit la région.

Pour les garçons, excepté le Centre-Est où les déclarations indiquent 20 ans, dans toutes les autres régions, c'est l'âge de 25 ans qui est avancé.

Parmi les adolescentes interrogées, 56,4\% pensent que l'âge au mariage du garçon selon le Code est acceptable. Le pourcentage varie par région, allant de 46,1\% au Centre-Nord à $66,4 \%$ à l'Est. 
Tableau 3.25 : Perceptions des adolescentes sur l'âge auquel les filles et les garçons se marient généralement ou peuvent se marier dans les régions (en \%)

\begin{tabular}{|c|c|c|c|c|c|c|}
\hline \multirow[t]{2}{*}{ Age en années } & \multicolumn{5}{|c|}{ Région } & \multirow[b]{2}{*}{ Ensemble } \\
\hline & Centre-Sud & Est & Sahel & Centre-Est & Centre-Nord & \\
\hline $\begin{array}{l}\text { Age auquel les filles se } \\
\text { marient généralement } \\
\text { (Mode) }\end{array}$ & 17 & 18 & 15 & 16 & 17 & 17 \\
\hline Pourcentage & 38 & 32,4 & 31 & 29,5 & 28,4 & 27,4 \\
\hline $\begin{array}{l}\text { Age auquel les garçons } \\
\text { se marient généralement } \\
\text { (Mode) }\end{array}$ & 20 & 20 & 20 & 20 & 20 & 20 \\
\hline Pourcentage & 31,8 & 37,9 & 51,2 & 49,4 & 33,1 & 40 \\
\hline $\begin{array}{l}\text { Age à partir duquel une } \\
\text { fille peut se marier } \\
\text { (Mode) }\end{array}$ & 20 & 20 & 20 & 20 & 20 & 20 \\
\hline Pourcentage & 30,2 & 33 & 36,9 & 27 & 27,2 & 30 \\
\hline $\begin{array}{l}\text { Age à partir duquel un } \\
\text { garçon peut se marier } \\
\text { (Mode) }\end{array}$ & 25 & 25 & 25 & 20 & 25 & 25 \\
\hline Pourcentage & 30,2 & 33,5 & 40,5 & 38,4 & 27,2 & 32,1 \\
\hline Effectif & 192 & 182 & 84 & 237 & 169 & 864 \\
\hline
\end{tabular}

N.B. : 34,6\% d'adolescentes n'ont pas pu donner un âge

D’une manière générale, moins de la moitié des adolescentes pensent que l'âge au mariage de la fille selon le code est acceptable. Les variations par région vont de $39 \%$ au Centre-Nord à $61,5 \%$ à l'Est.

Parmi les adolescentes interrogées, 56,4\% pensent que l'âge au mariage du garçon selon le Code est acceptable. Le pourcentage varie par région, allant de 46,1\% au Centre-Nord à $66,4 \%$ à l'Est.

Tableau 3.26 : Connaissances et perceptions par rapport à l'âge au mariage chez les adolescentes (en \%)

\begin{tabular}{|c|c|c|c|c|c|c|}
\hline \multirow{2}{*}{$\begin{array}{l}\text { Avez-vous entendu parler du Code des } \\
\text { Personnes et de la Famille }\end{array}$} & \multicolumn{6}{|c|}{ Région } \\
\hline & $\begin{array}{l}\text { Centre- } \\
\text { Sud }\end{array}$ & Est & Sahel & Centre-Est & Centre-Nord & Ensemble \\
\hline Oui & 6,8 & 4,5 & 34,8 & 5,9 & 5,8 & $\overline{8,3}$ \\
\hline Non & 92,8 & 95,5 & 56,3 & 70,4 & 89,9 & $\overline{83,6}$ \\
\hline NSP & 0,3 & 0,0 & 8,9 & 23,7 & 4,3 & 8,2 \\
\hline Total & 100,0 & 100,0 & 100,0 & 100,0 & 100,0 & 100,0 \\
\hline Effectif & 295 & 288 & 116 & 361 & 260 & 1320 \\
\hline \multicolumn{7}{|l|}{$\begin{array}{l}\text { Savez-vous que le code prévoit un âge } \\
\text { minimum de mariage pour les filles et les } \\
\text { garçons }\end{array}$} \\
\hline Oui & 65,0 & 42,9 & 80,0 & 69,6 & 50,0 & 65,8 \\
\hline Non & 15,0 & 50,0 & 10,0 & 17,4 & 25,0 & 19,7 \\
\hline NSP & 20,0 & 7,1 & 10,0 & 13,0 & 25,0 & 14,5 \\
\hline Total & 100,0 & 100,0 & 100,0 & 100,0 & 100,0 & 100,0 \\
\hline Effectif & 20 & 14 & 40 & 23 & 20 & 117 \\
\hline \multicolumn{7}{|l|}{$\begin{array}{l}\text { Appréciation de l'âge au mariage de la } \\
\text { fille selon le code }\end{array}$} \\
\hline Trop bas & 40,3 & 32,2 & 36,0 & 34,8 & 44,4 & 37,5 \\
\hline Acceptable & 47,1 & 61,5 & 59,5 & 42,9 & 39,0 & 48,5 \\
\hline Elevé & 0,3 & 0,0 & 0,0 & 4,7 & 0,4 & 1,5 \\
\hline
\end{tabular}




\begin{tabular}{lrrrrrr}
\hline Sans opinion & 12,3 & 6,3 & 4,5 & 17,5 & 16,2 & 12,5 \\
\hline Total & 100,0 & 100,0 & 100,0 & 100,0 & 100,0 & 100,0 \\
\hline Effectif & 295 & 288 & 116 & 361 & 260 & 1320 \\
\hline
\end{tabular}

\begin{tabular}{lrrrrrr}
\hline $\begin{array}{l}\text { Appréciation de l'Age au mariage du } \\
\text { garçon selon le code }\end{array}$ & & & & & & \\
\hline Trop bas & 34,8 & 26,2 & 41,1 & 20,1 & 36,0 & 29,7 \\
\hline Acceptable & 51,9 & 66,4 & 52,7 & 60,7 & 46,1 & 56,4 \\
\hline Elevé & 0,3 & 0,3 & 0,0 & 1,9 & 0,8 & 0,8 \\
\hline Sans opinion & 13,0 & 7,0 & 6,3 & 17,3 & 17,1 & 13,1 \\
\hline Total & 100,0 & 100,0 & 100,0 & 100,0 & 100,0 & 100,0 \\
\hline Effectif & 295 & 288 & 116 & 361 & 260 & 1320 \\
\hline
\end{tabular}

\section{Perceptions du mariage précoce}

A quel âge peut-on considérer qu'une fille s’est mariée très tôt (précocement)?

Sur l'ensemble des adolescentes enquêtées, 50\% déclarent qu'un mariage avant 15 ans peut être considéré comme un mariage précoce. Au Centre-Sud, au Centre-Nord et à l'Est, 50\% des adolescentes considèrent que le mariage est précoce pour la fille avant 15 ans alors qu'au Sahel et à l'Est, cet âge se situe avant 14 ans.

Tableau 3.27: Caractéristiques de tendance centrale et de dispersion de l’âge au mariage précoce (en \%)

\begin{tabular}{lrrrrrr}
\hline Caractéristiques & \multicolumn{7}{c}{ Région } & & \\
\hline & Centre-Sud & Est & Sahel & Centre-Est & Centre-Nord & Ensemble \\
\hline Mode & 15,0 & 15,0 & 15,0 & 10,0 & 15,0 & 15,0 \\
\hline Médiane & 15,0 & 15,0 & 14,0 & 14,0 & 15,0 & 15,0 \\
\hline Moyenne & 14,7 & 14,4 & 13,7 & 13,1 & 14,9 & 14,2 \\
\hline Ecart type & 2,2 & 1,6 & 1,7 & 2,3 & 2,1 & 2,2 \\
\hline Effectif & 262 & 266 & 105 & 301 & 215 & 1149 \\
\hline
\end{tabular}

En se référant au Code, le pourcentage de mariage précoce dans la zone de l'étude est de $37,2 \%$. Cependant, il varie de $8 \%$ au Centre-Nord à 74,1\% au Sahel en passant par $39,1 \%$ à l'Est et $45,2 \%$ au Centre-Est. Etant donné que les âges au mariage sont basés sur la simple déclaration des adolescentes, il se pourrait que le nombre de mariages précoces soit sousestimé pour certaines régions. Les données font ressortir que 10,2\% (14 sur 137) des mariages des adolescentes ont lieu entre 10 et 14 ans et la quasi-totalité de ces mariages sont enregistrés au Sahel (13 mariages).

Tableau 3.28 : Pourcentage de mariage précoce (âge au premier mariage avant 17 ans) par région

\begin{tabular}{lrrrrrr}
\hline & Centre-Sud & Est & Sahel & Centre-Est & Centre-Nord & Ensemble \\
\hline $\begin{array}{l}\text { Mariages } \\
\text { précoces }^{5}\end{array}$ & 6 & 9 & 20 & 14 & 2 & 51 \\
\hline Mariages $^{6}$ & 31 & 23 & 27 & 31 & 25 & 137 \\
\hline Pourcentage & 19,3 & 39,1 & 74,1 & 45,2 & 8,0 & 37,2 \\
\hline
\end{tabular}

\footnotetext{
${ }^{5}$ Mariage ayant eu lieu entre 10 et 16 ans

${ }^{6}$ Mariage ayant eu entre 10 et 19 ans
} 


\section{Age souhaité au mariage}

Pour les adolescentes non encore mariées, 4,7\% d'entre elles souhaiteraient se marier avant l'âge de 17 ans alors que la majorité (26,4\%) souhaiterait l'âge de 20 ans et $13 \%$ l'âge de 25 ans. L'âge minimum souhaité au mariage est de 15 ans alors que l'âge maximal se situe à 30 ans. $13,6 \%$ de l'ensemble des adolescentes ne savent pas l'âge auquel elles souhaiteraient se marier.

Excepté le Centre-Nord où 50\% des adolescentes célibataires souhaiteraient se marier avant 21 ans, dans les quatre autres régions, $50 \%$ des adolescentes célibataires le feraient avant 20 ans.

Tableau 3.29 : Caractéristiques de tendance centrale et de dispersion de l'âge souhaité au mariage chez les adolescentes célibataires (en \%)

\begin{tabular}{lrrrrrr}
\hline Caractéristiques & \multicolumn{7}{c}{ Région } \\
\cline { 2 - 8 } & Centre-Sud & Est & Sahel & Centre-Est & Centre-Nord & Ensemble \\
\hline Mode & 20,0 & 20,0 & 20,0 & 20,0 & 20,0 & 20,0 \\
\hline Médiane & 20,0 & 20,0 & 20,0 & 20,0 & 21,0 & 20,0 \\
\hline Moyenne & 21,2 & 21,4 & 20,4 & 20,8 & 21,9 & 21,2 \\
\hline Ecart type & 3,6 & 3,3 & 2,7 & 3,4 & 2,9 & 3,3 \\
\hline Effectif & 214 & 213 & 73 & 258 & 202 & 960 \\
\hline
\end{tabular}

A la question «A quel âge auriez-vous souhaité vous marier ?», 50\% des adolescentes mariées auraient souhaité se marier avant 17 ans au Sahel et avant 19 ans dans les autres régions. Le choix de cet âge est essentiellement personnel selon la grande majorité des adolescentes $(68,8 \%)$.

Tableau 3.30 : Caractéristiques de tendance centrale et de dispersion de l'âge souhaité au mariage chez les adolescentes mariées (en \%)

\begin{tabular}{lrrrrrr}
\hline Caractéristiques & \multicolumn{7}{c}{ Région } \\
\cline { 2 - 7 } & Centre-Sud & Est & Sahel & Centre-Est & Centre-Nord & Ensemble \\
\hline Mode & 19,0 & 17,0 & 17,0 & 20,0 & 17,0 & 17,0 \\
\hline Médiane & 19,0 & 18,0 & 17,0 & 19,0 & 18,5 & 19,0 \\
\hline Moyenne & 19,1 & 18,6 & 17,5 & 18,5 & 18,4 & 18,4 \\
\hline Ecart type & 1,9 & 2,0 & 1,7 & 2,4 & 1,7 & 2,1 \\
\hline Effectif & 32 & 21 & 24 & 30 & 24 & 131 \\
\hline
\end{tabular}

Selon 69,5\% des adolescentes enquêtées, c'est la jeune fille elle-même qui doit choisir son futur époux. Pour le choix de la future épouse d'un homme, 73,3\% d'entre elles affirment que c'est le jeune homme qui doit décider lui-même.

Tableau 3.31 : Personne décidant du choix du futur époux de la fille ou de la future épouse de l'homme (en \%)

\begin{tabular}{lrrrrrr}
\hline $\begin{array}{l}\text { Qui décide du choix du } \\
\text { futur époux d'une fille? }\end{array}$ & Centre-Sud & Est & Sahel & Centre-Est & Centre-Nord & Ensemble \\
\hline Père & 15,1 & 14,7 & 19,6 & 27,9 & 11,6 & 18,2 \\
\hline Mère & 0,3 & 0,3 & 4,5 & 0,6 & 1,2 & 0,9 \\
\hline Les deux & 10,6 & 5,2 & 11,6 & 0,3 & 6,6 & 5,9 \\
\hline $\begin{array}{l}\text { Autres membres de } \\
\text { famille }\end{array}$ & 0,3 & 0,3 & 3,6 & 0,3 & 0,8 & 0,7 \\
\hline Jeune fille elle-même & 67,5 & 76,2 & 58,9 & 69,1 & 69,4 & 69,5 \\
\hline Autre & 0,7 & 0,0 & 0,0 & 0,3 & 1,2 & 0,5 \\
\hline Ne sait pas & 5,5 & 3,1 & 1,8 & 1,7 & 9,3 & 4,4 \\
\hline Total & 100,0 & 100,0 & 100,0 & 100,0 & 100,0 & 100,0 \\
\hline Effectif & 295 & 288 & 116 & 361 & 260 & 1320 \\
\hline
\end{tabular}




\begin{tabular}{|c|c|c|c|c|c|c|}
\hline $\begin{array}{l}\text { Qui décide du choix de } \\
\text { la future épouse d'un } \\
\text { homme }\end{array}$ & & & & & & \\
\hline Père & 11,4 & 11,9 & 15,2 & 23,9 & 9,5 & 14,9 \\
\hline Mère & 0,3 & 1,0 & 5,4 & 0,6 & 0,0 & 0,9 \\
\hline Les deux & 9,3 & 4,5 & 10,7 & 0,0 & 6,3 & 5,2 \\
\hline $\begin{array}{l}\text { Autres membres de la } \\
\text { famille }\end{array}$ & 0,7 & 0,0 & 2,7 & 0,3 & 0,8 & 0,6 \\
\hline Jeune homme lui-même & 71,7 & 79,0 & 64,3 & 73,6 & 72,3 & 73,3 \\
\hline Autre & 0,7 & 0,0 & 0,0 & 0,3 & 0,4 & 0,3 \\
\hline Ne sait pas & 5,9 & 3,5 & 1,8 & 1,4 & 10,7 & 4,7 \\
\hline Total & 100,0 & 100,0 & 100,0 & 100,0 & 100,0 & 100,0 \\
\hline Effectif & 295 & 288 & 116 & 361 & 260 & 1320 \\
\hline
\end{tabular}

\subsubsection{Canaux d'information chez les chefs de ménage}

A la question de savoir comment les chefs de ménage s’informent sur les évènements du pays et du monde. Il ressort dans les cinq régions concernées par l'étude que la radio est le canal le plus utilisé (plus de 50\%). Ensuite suivent les causeries familiales et la télévision.

C'est dans le Centre-Est que la plus grande proportion des chefs de ménages $(66,6 \%)$ s'informe principalement par la radio. Ces proportions sont de 54,5\% pour le Centre-Nord, 43,7\% dans le Centre-Sud, 40,6\% dans le Sahel et de 37,3\% dans la région de l’Est.

Les causeries avec les amis ou en famille constituent le premier canal d'information sur les évènements du pays et du monde le plus utilisé dans la région de l'Est. Ce canal d'information vient en seconde position après la radio dans la région du Centre-Sud $(33,1 \%)$. Dans le Sahel, le Centre-Est et le Centre-Nord par contre, la télévision constitue le second canal d’information principalement utilisé.

Tableau 3.32: Répartition des chefs de ménage selon le canal d’information le plus utilisé (en \%)

\begin{tabular}{lrrrrrr}
\hline Canal d'information le plus utilisé & \multicolumn{7}{c}{ Région } \\
\cline { 2 - 7 } & Centre-Sud & Est & Sahel & Centre-Est & Centre-Nord & Ensemble \\
\hline Radio & 43,7 & 37,3 & 40,6 & 66,6 & 54,5 & 50,9 \\
\hline Télévision & 22,7 & 20,2 & 42,8 & 16,4 & 31,0 & 23,7 \\
\hline Journaux & 0,3 & 0,8 & 0,0 & 0,2 & 0,0 & 0,3 \\
\hline Conversation entre amis/famille & 33,1 & 40,9 & 16,7 & 12,9 & 14,2 & 23,8 \\
\hline Ecole & 0,0 & 0,8 & 0,0 & 0,0 & 0,0 & 0,2 \\
\hline Autre & 0,3 & 0,0 & 0,0 & 3,9 & 0,3 & 1,2 \\
\hline Total & 100,0 & 100,0 & 100,0 & 100,0 & 100,0 & 100,0 \\
\hline Effectif & 359 & 367 & 138 & 488 & 348 & 1700 \\
\hline
\end{tabular}




\subsection{Vécu du mariage chez les adolescentes}

\subsubsection{Contexte socio-familial des adolescentes}

\section{Caractéristiques de l'équipement et de l'habitat des ménages}

Lors de l'enquête ménage, l'on s'est intéressé à certaines caractéristiques du logement (disponibilité de l'électricité, approvisionnement en eau, matériaux du toit et revêtement du sol). On a également recueilli des informations sur la possession d'un certain nombre d’équipements modernes (radio, télévision, réfrigérateur, bicyclette, motocyclette, véhicule). Ces caractéristiques permettent d’apprécier les conditions socio-économiques des ménages. Ce sont, en outre, des éléments qui entrent en compte dans l'amélioration des conditions de vie et partant dans l'amélioration de l'état de santé et du bien-être de la population et permettent par ailleurs de comprendre un certain nombre d'attitudes sur le mariage précoce et le vécu des adolescentes.

\section{Equipement et moyens de transport}

La possession des biens de consommation durables par les ménages est révélatrice de leur niveau de vie et de leur accessibilité à certains services sociaux de base. Les questions posées sur les biens de consommation durables des ménages ont porté sur la possession d'un poste radio, d'une télévision, d'un téléphone ainsi que sur certains moyens de transport tels qu'une bicyclette, une motocyclette et une voiture.

Sur l'ensemble des ménages enquêtés, $82 \%$ possèdent une radio. Cette proportion est de plus de $87 \%$ au Sahel, au Centre-Est et au Centre-Nord. C'est dans la région de l'Est $(71,7 \%)$ qu'on a enregistré la proportion la plus faible.

Cependant, la possession d'un poste téléviseur et d'un réfrigérateur n'est pas la chose la plus couramment partagée par les ménages. En effet, 39,5\% et seulement 12,2\% des ménages possèdent respectivement un poste téléviseur et un réfrigérateur.

Pour ce qui est du téléphone (fixe ou mobile), il ressort que 65,5\% des ménages le possèdent. Cette proportion varie entre 53,8\% dans le Centre-Sud et de 70,8\% à l’Est.

Tableau 3.33 : Possession d'équipement par les ménages (en \%)

\begin{tabular}{lrrrrrr}
\hline $\begin{array}{l}\text { Possession de } \\
\text { d'équipement }\end{array}$ & biens & \multicolumn{7}{c}{ Région } \\
\cline { 2 - 8 } & Centre-Sud & Est & Sahel & Centre-Est & Centre-Nord & Ensemble \\
\hline Une radio & 77,0 & 71,7 & 87,0 & 87,7 & 88,1 & 82,0 \\
\hline Une télévision & 29,7 & 31,9 & 50,0 & 40,6 & 52,2 & 39,5 \\
\hline Un réfrigérateur & 11,2 & 9,5 & 13,8 & 11,3 & 16,5 & 12,2 \\
\hline $\begin{array}{l}\text { Un téléphone (fixe ou } \\
\text { mobile) }\end{array}$ & 53,8 & 70,8 & 68,8 & 66,0 & 69,9 & 65,5 \\
\hline Effectif & 359 & 367 & 138 & 488 & 348 & 1700 \\
\hline
\end{tabular}

En ce qui concerne les moyens de transport dont disposent les ménages, il ressort des résultats du tableau ci-dessous que le vélo est le moyen de transport que possède le plus grand nombre de ménages $(83,5 \%)$, ensuite la mobylette $(36,0 \%)$ et la moto $(22,3 \%)$. Seulement une proportion très réduite de ménages possède une voiture $(3,1 \%)$.

Le moyen le plus utilisé pour effectuer les transports par les différents membres du ménage est le vélo dans presque toutes les régions. La proportion de ménages se servant du vélo comme moyen de transport principal est de 65\% dans le Centre-Sud, 61\% dans l'Est et 32,6\% au Sahel. 
Tableau 3.34 : Possession des moyens de transport (\%)

\begin{tabular}{|c|c|c|c|c|c|c|}
\hline \multirow[t]{2}{*}{ Possession de biens de consommation } & \multicolumn{6}{|c|}{ Région } \\
\hline & Centre-Sud & Est & Sahel & Centre-Est & Centre-Nord & Ensemble \\
\hline Possession de vélo dans le ménage & 89,4 & 86,4 & 49,3 & 85,4 & 85,5 & 83,5 \\
\hline Possession de mobylette dans le ménage & 24,9 & 21,3 & 31,2 & 50,1 & 45,2 & 36,0 \\
\hline Possession de Moto dans le ménage & 26,1 & 28,9 & 36,2 & 6,2 & 28,7 & 22,3 \\
\hline Possession de voiture dans le ménage & 1,7 & 4,6 & 2,9 & 2,1 & 4,6 & 3,1 \\
\hline Effectif & 359 & 367 & 138 & 488 & 348 & 1700 \\
\hline \multicolumn{7}{|l|}{$\begin{array}{l}\text { Principal moyen de transport utilisé par } \\
\text { les membres du ménage }\end{array}$} \\
\hline Aucun & 5,6 & 9,0 & 23,9 & 4,6 & 7,6 & 8,0 \\
\hline Vélo & 65,0 & 61,0 & 32,6 & 47,1 & 55,7 & 54,5 \\
\hline Mobylette & 17,9 & 13,9 & 20,3 & 44,7 & 20,7 & 25,4 \\
\hline Moto & 9,8 & 16,1 & 17,4 & 2,9 & 14,0 & 10,7 \\
\hline Voiture & 0,6 & 0,0 & 0,7 & 0,4 & 0,0 & 0,3 \\
\hline Autre & 1,1 & 0,0 & 5,1 & 0,2 & 2,0 & 1,1 \\
\hline Total & 100,0 & 100,0 & 100,0 & 100,0 & 100,0 & 100,0 \\
\hline Effectif & 359 & 367 & 138 & 488 & 348 & 1700 \\
\hline
\end{tabular}

\section{Caractéristiques des logements}

Lors de l'enquête ménage, on s'est intéressé à certaines caractéristiques du logement (disponibilité de l'électricité, approvisionnement en eau, types de murs, matériaux de toiture et nombre de pièces utilisées pour dormir). Ces caractéristiques peuvent renseigner sur les conditions de vie des adolescentes dans leur ménage.

Il ressort du tableau ci-dessous que 42,8\% des ménages enquêtés disposent de l'électricité. C'est dans le Sahel qu'on enregistre la plus faible proportion des ménages disposant de l'électricité alors que dans le Centre-Nord plus de la moitié $(57,7 \%)$ des ménages en disposent.

L'eau de boisson est déterminante dans l'état sanitaire de la population. La consommation de l'eau non potable est à l'origine de plusieurs maladies hydriques : diarrhée, dracunculose, etc. Concernant la source de l'eau utilisée pour la consommation, on constate que plus d'un ménage sur deux (50,4 \%) utilisent l'eau des puits et des forages publics, 37,6 \% des ménages utilisent l'eau des robinets publics, 10,8 \% disposent de l'eau courante à domicile. L'eau des rivières est encore utilisée par 1,1 \% des ménages enquêtés. Dans la région du Centre-Sud, 3,4\% des ménages utilisent encore l'eau des marigots comme principale source d'approvisionnement.

Au cours de l'enquête ménage, on s'est également intéressé au principal combustible utilisé pour la cuisine dans les ménages. Il ressort que le bois est principalement utilisé $(86,5 \%)$ et le charbon de bois pour seulement 2,4\% des ménages.

Le type de murs des maisons dans la plupart des ménages est en banco (35,3\%) ou en banco amélioré pour 37,3\% des cas. Au Sahel, on note la prédominance du banco (66,7\%) et à l'Est, le banco amélioré $(63,5 \%)$.

Les maisons dans la majorité des ménages enquêtés sont recouvertes par les tôles ondulées (72,5\%). La paille est utilisée pour 17,9\% des cas mais plus fréquent dans le Centre-Sud (42,3\%), l'Est (16,6\%) et Centre-Est (15,6\%). 
Concernant le type de toilettes dont disposent les ménages, les résultats montrent que trois ménages enquêtés sur dix ne possèdent pas de toilettes dans leur logement (29,6\%). Cette proportion varie d'un maximum de $42 \%$ dans la région de l'Est à 8,4\% dans le Centre-Nord. Par ailleurs, 30,1\% des ménages disposent de fosses ou de latrines rudimentaires et $33,7 \%$ de toilettes améliorées.

Tableau 3.35 : Possession d'électricité et la principale source d'approvisionnement en eau de boisson des ménages par région (en \%)

\begin{tabular}{lrrrrrr}
\hline Electricité & \multicolumn{7}{c}{ Région } \\
\cline { 2 - 8 } & Centre-Sud & \multicolumn{1}{c}{ Est } & \multicolumn{1}{c}{ Sahel } & Centre-Est & Centre-Nord & Ensemble \\
\hline Oui & 31,7 & 27,8 & 49,3 & 49,8 & 57,7 & 42,8 \\
\hline Non & 68,3 & 72,2 & 50,7 & 50,2 & 42,3 & 57,2 \\
\hline Total & 100,0 & 100,0 & 100,0 & 100,0 & 100,0 & 100,0 \\
\hline Effectif & 357 & 367 & 138 & 486 & 343 & 1691 \\
\hline $\begin{array}{l}\text { Principale source } \\
\text { d'approvisionnement en } \\
\text { eau de boisson }\end{array}$ & & & & & & \\
\hline Puits, forage public & & & & & & \\
\hline Robinet public & 67,4 & 65,5 & 19,9 & 36,5 & 48,1 & 50,4 \\
\hline Robinet dans la maison & 19,4 & 31,0 & 61,8 & 51,3 & 34,8 & 37,6 \\
\hline Marigot & 9,4 & 2,2 & 16,9 & 12,0 & 17,1 & 10,8 \\
\hline Autre & 3,4 & 1,4 & 1,5 & 0,0 & 0,0 & 1,1 \\
\hline Total & 0,3 & 0,0 & 0,0 & 0,2 & 0,0 & 0,1 \\
\hline Effectif & 100,0 & 100,0 & 100,0 & 100,0 & 100,0 & 100,0 \\
\hline
\end{tabular}

\section{Construction d'un proxy de niveau de vie des ménages}

En plus de la description de l'équipement et de l'habitat des ménages enquêtés, il a été procédé à la construction d'un indicateur composite de niveau de vie qui prend en compte les caractéristiques de l'équipement et de l'habitat. Ainsi, les adolescentes se repartissent entre trois catégories de niveau de vie (faible, moyen et élevé).

Les ménages de niveau de vie élevé (riche) sont caractérisés par un pourcentage plus élevé dans la possession des biens ou équipements suivants : radio (100\%), téléphone (100\%), mobylette (100\%), télévision (50\%), électricité (50\%), des maisons en dur (50\%), latrines améliorée (50\%). Par contre les ménages de niveau de vie faible (pauvre) sont caractérisés par une faible possession des biens ou équipements suivants : radio (68,3\%), téléphone (31,7\%), télévision (7,3\%), mobylette $(4,9 \%)$, latrine améliorée $(0 \%)$ et maisons en dur $(0 \%)$. Les ménages de niveau de vie moyen occupent une position intermédiaire entre les catégories précédentes (voir tableau A25 en annexe).

Il ressort que la majorité des adolescentes sont issues de ménages pauvres (69,5\%). Celles des ménages de niveau de vie moyen représentent $27,1 \%$ contre seulement $3,4 \%$ issues de ménages de niveau de vie élevé. 
Graphique 3.7 : Répartition des adolescentes selon le niveau du ménage

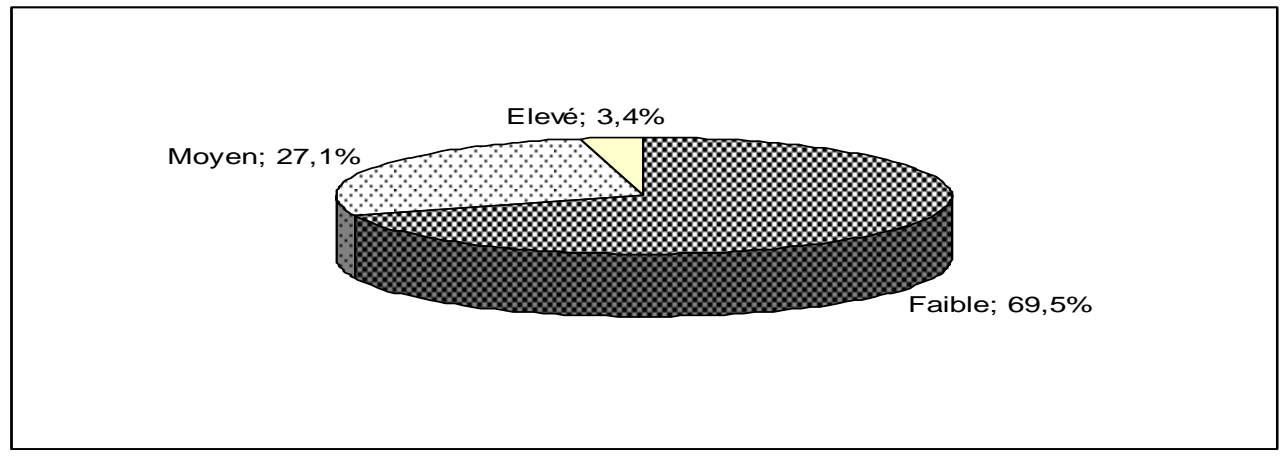

\subsubsection{Vécu des adolescentes}

\section{Grossesses chez les adolescentes}

Seulement $10 \%$ des adolescentes ont déjà eu au moins une grossesse. La majorité des adolescentes (8,9\%) est à leur première grossesse alors que $1,7 \%$ et $0,2 \%$ sont respectivement à leur deuxième et troisième grossesse.

La proportion des adolescentes ayant déjà eu une grossesse est faible dans toutes les régions avec une proportion variant entre $6,6 \%$ et $12,9 \%$.

Tableau 3.36 : Répartition des adolescentes selon le nombre de grossesses déjà eu (en \%)

\begin{tabular}{lrrrrrr}
\hline Combien de grossesses & \multicolumn{7}{c}{ Région } \\
\cline { 2 - 8 } avez-vous déjà eu ? & \multicolumn{1}{c}{ Centre-Sud } & \multicolumn{1}{c}{ Est } & \multicolumn{1}{c}{ Sahel } & Centre-Est & Centre-Nord & Ensemble \\
\hline 0 & 87,1 & 89,6 & 85,8 & 93,3 & 87,3 & 89,3 \\
\hline 1 & 11,2 & 9,4 & 8,8 & 5,8 & 10,0 & 8,9 \\
\hline 2 & 1,4 & 0,7 & 5,3 & 0,8 & 2,7 & 1,7 \\
\hline 3 & 0,3 & 0,3 & 0,0 & 0,0 & 0,0 & 0,2 \\
\hline Total & 100,0 & 100,0 & 100,0 & 100,0 & 100,0 & 100,0 \\
\hline Effectif & 295 & 288 & 113 & 359 & 260 & 1315 \\
\hline
\end{tabular}

\section{Considérations spécifiques des adolescentes mariées}

Sauf au Centre-Nord où 55,6\% des déclarations indiquent que c'est la jeune fille elle-même qui a fait le choix de son conjoint, d'une manière générale, l'avis des parents seuls ou en accord avec la jeune fille jouent un rôle important.

Dans la majorité des cas (93\%), la jeune fille affirme avoir approuvé ce choix.

Tableau 3.37 : Choix du conjoint chez les adolescentes mariées (en \%)

\begin{tabular}{|c|c|c|c|c|c|c|}
\hline \multirow{2}{*}{$\begin{array}{l}\text { Qui a fait le choix de votre } \\
\text { conjoint? }\end{array}$} & \multicolumn{6}{|c|}{ Région } \\
\hline & Centre-Sud & Est & Sahel & Centre-Est & Centre-Nord & Ensemble \\
\hline Parents/famille seuls & 15,2 & 0 & 37 & 33,3 & 25,9 & 23,1 \\
\hline $\begin{array}{l}\text { Parents/famille avec l'accord du } \\
\text { sujet }\end{array}$ & 15,2 & 0 & 33,3 & 9,1 & 0 & 11,9 \\
\hline $\begin{array}{l}\text { Vous-même avec accord de la } \\
\text { famille }\end{array}$ & 27,3 & 82,6 & 22,2 & 12,1 & 18,5 & 30,1 \\
\hline $\begin{array}{l}\text { Vous-même sans l'influence de } \\
\text { la famille }\end{array}$ & 39,4 & 17,4 & 7,4 & 45,5 & 55,6 & 34,3 \\
\hline Ne sait pas & 3 & 0 & 0 & 0 & 0 & 0,7 \\
\hline Total & 100 & 100 & 100 & 100 & 100 & 100 \\
\hline Effectif & 33 & 23 & 27 & 33 & 27 & 143 \\
\hline
\end{tabular}




\begin{tabular}{lrrrrrr}
\hline Avez-vous approuvé ce choix? & & & & & & \\
\hline Oui & 100 & 100 & 74,1 & 90,9 & 100 & 93 \\
\hline Non & 0 & 0 & 25,9 & 9,1 & 0 & 7 \\
\hline Total & 100 & 100 & 100 & 100 & 100 & 100 \\
\hline Effectif & 33 & 23 & 27 & 33 & 27 & 143 \\
\hline
\end{tabular}

D’après le graphique 3.8, il ressort que les proportions d'adolescentes mariées dont le choix du mari a été fait par la jeune fille en accord avec la famille ne diffèrent pas selon le milieu de résidence (29\% en milieu rural contre $30,9 \%$ en milieu urbain). Il en est de même lorsque pour les adolescentes mariées dont le choix du conjoint a été fait par les parents/famille seuls (22,6\% en milieu rural contre $23,5 \%$ en milieu urbain).

Cependant, pour 37\% d'adolescentes mariées en milieu urbain contre 30,6\% en milieu rural, le choix du conjoint a été opéré par l'adolescente elle-même sans l'influence de la famille. A l'inverse, pour $17,7 \%$ d'adolescentes mariées en milieu rural contre 7,4\% en milieu urbain, le choix du conjoint a été fait par les parents ou la famille avec l'accord de l'adolescente.

Graphique 3.8 : Choix du conjoint chez les adolescentes mariées selon le milieu de résidence (en \%)

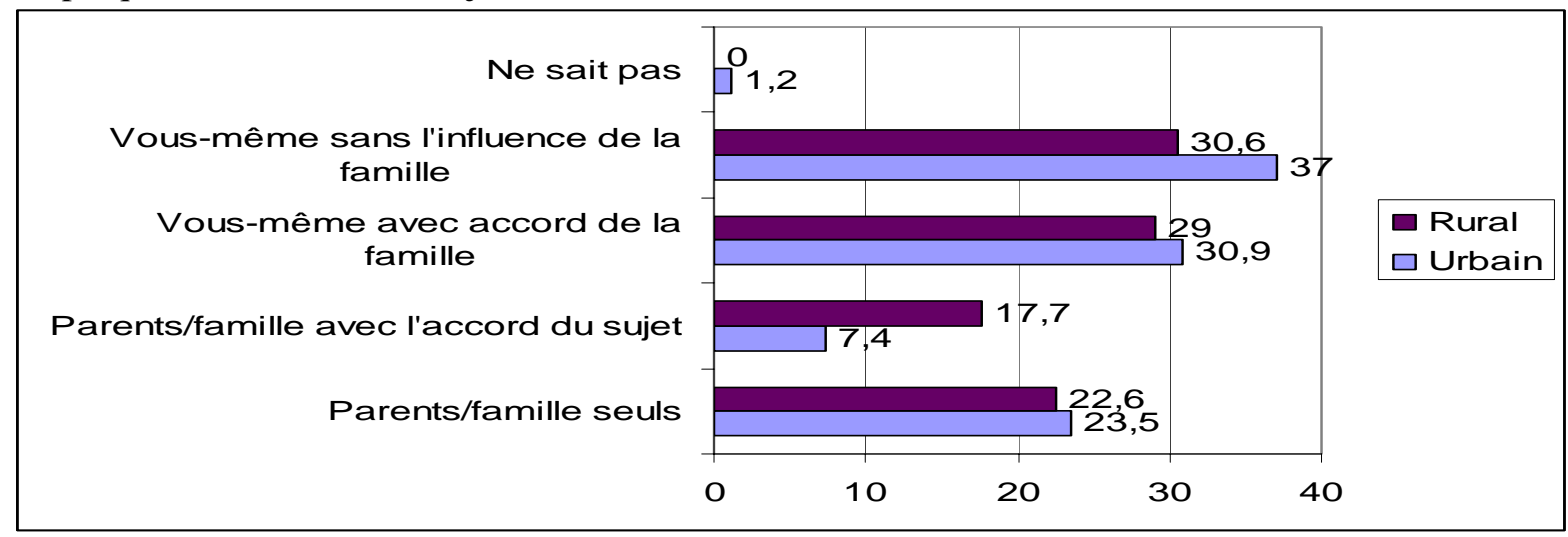

\section{Age au premier mariage}

La majorité des adolescentes mariées l'ont été à l'âge de 17 ans. C'est dans le Sahel que la plupart des adolescentes se sont mariées précocement avec un âge moyen au premier mariage de 15 ans alors que dans les autres régions, cet âge est de 17 ans.

Une forte proportion des adolescentes (73\%) trouve que leur âge au premier mariage est convenable.

Tableau 3.38: Caractéristiques de tendance centrale et de dispersion de l'âge des adolescentes au premier mariage (en \%)

\begin{tabular}{|c|c|c|c|c|c|c|}
\hline \multirow[t]{2}{*}{ Caractéristiques } & \multicolumn{3}{|c|}{ Région } & \multirow[b]{2}{*}{ Centre-Est } & \multirow[b]{2}{*}{ Centre-Nord } & \multirow[b]{2}{*}{ Ensemble } \\
\hline & Centre-Sud & Est & Sahel & & & \\
\hline Mode & 17,0 & 16,0 & 14,0 & 17,0 & 17,0 & 17,0 \\
\hline Médiane & 17,0 & 17,0 & 15,0 & 17,0 & 17,0 & 17,0 \\
\hline Moyenne & 17,1 & 16,9 & 15,1 & 16,6 & 17,4 & 16,6 \\
\hline Ecart type & 0,7 & 0,9 & 1,9 & 1,2 & 0,7 & 1,4 \\
\hline Effectif & 33 & 23 & 27 & 33 & 27 & 143 \\
\hline \multicolumn{7}{|l|}{$\begin{array}{l}\text { Votre } \\
\text { appréciation }\end{array}$} \\
\hline Trop tôt & 9,4 & 34,8 & 42,3 & 29,0 & 11,1 & 24,5 \\
\hline
\end{tabular}




\begin{tabular}{lrrrrrr}
\hline Convenable & 87,5 & 65,2 & 57,7 & 64,5 & 85,2 & 72,7 \\
\hline Sans opinion & 3,1 & 0,0 & 0,0 & 6,5 & 3,7 & 2,9 \\
\hline Total & 100,0 & 100,0 & 100,0 & 100,0 & 100,0 & 100,0 \\
\hline Effectif & 33 & 23 & 27 & 33 & 27 & 143 \\
\hline
\end{tabular}

\section{Type de cohabitation}

La majorité des adolescentes mariées ont déclaré vivre avec leur conjoint (92,1\%) alors que pour 7,9\% ne vivent pas avec leur conjoint. La plupart de ces jeunes couples vivent avec d'autres membres de leurs familles (64,6\%). Dans le Sahel, la quasi-totalité des couples d'adolescentes mariées (90,9\%) vivent avec d'autres membres de la famille. Ces membres sont généralement les parents biologiques du conjoint $(65,9 \%)$ ou les membres de la grande famille du conjoint (28\%).

Tableau 3.39 : Type de cohabitation chez les adolescentes mariées (en \%)

\begin{tabular}{|c|c|c|c|c|c|c|}
\hline \multirow{2}{*}{$\begin{array}{l}\text { Le couple vit-il } \\
\text { ensemble? }\end{array}$} & \multicolumn{6}{|c|}{ Région } \\
\hline & Centre-Sud & Est & Sahel & Centre-Est & Centre-Nord & Ensemble \\
\hline Oui & 93,9 & 90,9 & 88,5 & 90,3 & 96,3 & 92,1 \\
\hline Non & 6,1 & 9,1 & 11,5 & 9,7 & 3,7 & 7,9 \\
\hline Total & 100,0 & 100,0 & 100,0 & 100,0 & 100,0 & 100,0 \\
\hline Effectif & 33 & 22 & 26 & 31 & 27 & 139 \\
\hline \multicolumn{7}{|l|}{$\begin{array}{l}\text { Le couple vit-il avec } \\
\text { d'autres parents? }\end{array}$} \\
\hline Oui & 74,2 & 35,0 & 90,9 & 46,4 & 73,1 & 64,6 \\
\hline Non & 25,8 & 65,0 & 9,1 & $\overline{53,6}$ & 26,9 & 35,4 \\
\hline Total & 100,0 & 100,0 & 100,0 & 100,0 & 100,0 & 100,0 \\
\hline Effectif & 31 & 20 & 22 & 28 & 26 & 127 \\
\hline \multicolumn{7}{|l|}{ Avec qui vit-il ? } \\
\hline $\begin{array}{ll}\text { Parents } & \text { biologiques } \\
\text { du mari } & \\
\end{array}$ & 56,5 & 85,7 & 66,7 & 58,3 & 73,7 & 65,9 \\
\hline Parents de la femme & 0,0 & 0,0 & 4,8 & 0,0 & 0,0 & 1,2 \\
\hline $\begin{array}{l}\text { Autres parents/Grande } \\
\text { famille du mari }\end{array}$ & 39,1 & 14,3 & 28,6 & 16,7 & 26,3 & 28,0 \\
\hline Autre & 4,3 & 0,0 & & 25,0 & 0,0 & 4,9 \\
\hline Total & 100,0 & 100,0 & 100,0 & 100,0 & 100,0 & 100,0 \\
\hline Effectif & 23 & 7 & 21 & 12 & 19 & 82 \\
\hline
\end{tabular}

\section{Conseillers des adolescentes sur la vie en couple}

La majorité des adolescentes déclarent avoir reçu des conseils sur la vie du couple pendant le mariage, sauf au Centre-Est où cela est moins fréquent. Les personnes qui donnent ces conseils varient par ordre d'importance, d'une région à l'autre. Mais dans la majorité des cas, la mère constitue la personne centrale. Au Centre-Est, il s'agit de la mère et des beaux parents. Au Centre-Sud, ce sont les beaux parents, la mère et les autres parents. A l'Est, c'est la mère et les autres parents. Au Centre-Nord, ce sont les beaux-parents, les autres parents et la mère. Au Sahel, il s'agit des beaux-parents, des autres parents et de la mère. Elles déclarent recevoir les conseils avant et après le mariage. 
Tableau 3.40 : Les conseillers des adolescentes sur la vie de couple (en \%)

\begin{tabular}{lrrrrrrr}
\hline $\begin{array}{l}\text { Avoir eu conseils avant } \\
\text { mariage }\end{array}$ & le & \multicolumn{7}{c}{ Région } \\
\cline { 2 - 8 } & \multicolumn{2}{c}{ Centre-Sud } & Est & \multicolumn{1}{c}{ Sahel } & Centre-Est & Centre-Nord & Ensemble \\
\hline Oui & 81,3 & 43,5 & 96,3 & 48,4 & 77,8 & 70 \\
\hline Non & 18,8 & 56,5 & 3,7 & 51,6 & 22,2 & 30 \\
\hline Total & 100 & 100 & 100 & 100 & 100 & 100 \\
\hline Effectif & 33 & 23 & 27 & 33 & 27 & 143 \\
\hline
\end{tabular}

\begin{tabular}{lrrrrrr}
\hline $\begin{array}{l}\text { La personne ayant donné les } \\
\text { conseils }\end{array}$ & \multicolumn{1}{l}{} \\
\hline Mère & 88,5 & 81,8 & 69,2 & 73,3 & 95 & 81,6 \\
\hline Père & 0 & 0 & 7,7 & 0 & 0 & 2 \\
\hline Baux parents & 0 & 0 & 0 & 13,3 & 5 & 3,1 \\
\hline Autres parents & 3,8 & 18,2 & 19,2 & 6,7 & 0 \\
\hline Conseillers religieux & 3,8 & 0 & 0 & 6,7 & 0 \\
\hline Autre & 3,8 & 0 & 3,8 & 0 & 0 \\
\hline Total & 100 & 100 & 100 & 100 & 100 \\
\hline Effectif & 26 & 11 & 26 & 15 & 20 \\
\hline
\end{tabular}

\begin{tabular}{|c|c|c|c|c|c|c|}
\hline $\begin{array}{l}\text { Avoir des conseils pendant le } \\
\text { mariage }\end{array}$ & & & & & & \\
\hline Non & 37,5 & 21,7 & 14,8 & 61,3 & 37 & 35,7 \\
\hline Oui & 62,5 & 78,3 & 85,2 & 38,7 & 63 & 64,3 \\
\hline Total & 100 & 100 & 100 & 100 & 100 & 100 \\
\hline Effectif & 33 & 23 & 27 & 33 & 27 & 143 \\
\hline \multicolumn{7}{|l|}{ Personne donnant ces conseils } \\
\hline Mère & 25 & 71,4 & 6,7 & 75 & 16,7 & 29,5 \\
\hline Père & 0 & 0 & 0 & 0 & 16,7 & 2,3 \\
\hline Beaux parents & 33,3 & 0 & 73,3 & 25 & 50 & 43,2 \\
\hline Autres parents & 25 & 28,6 & 20 & 0 & 16,7 & 20,5 \\
\hline Autre & 16,7 & 0 & 0 & 0 & 0 & 4,5 \\
\hline Total & 100 & 100 & 100 & 100 & 100 & 100 \\
\hline Effectif & 12 & 7 & 15 & 4 & 6 & 44 \\
\hline
\end{tabular}

\section{Manquements dans le couple}

Les différents manquements du mari à ses devoirs d'époux cités par les adolescentes mariées sont par ordre d'importance le manque d'affection (60\%), l'absence de vie de couple (50\%) le manque de respect (45\%), l'infidélité dans le couple (40,7\%). Lorsque ces actes sont posés par un conjoint, la quasi-totalité des adolescentes du Centre-Sud et à l'Est n'ont eu aucun recours, et celles des régions du Sahel, du Centre-Est et du Centre affirment avoir eu recours à une structure ou à des personnes pour régler le problème.

Tableau 3.41 : Manquement du mari à ses devoirs d’époux (en \%)

\begin{tabular}{|c|c|c|c|c|c|c|}
\hline \multirow{2}{*}{$\begin{array}{l}\text { Manquement du mari à } \\
\text { ses devoirs d'époux }\end{array}$} & \multicolumn{6}{|c|}{ Région } \\
\hline & Centre-Sud & Est & Sahel & Centre-Est & Centre-Nord & Total \\
\hline Oui & 21,9 & 17,4 & 18,5 & 19,4 & 11,1 & 17,9 \\
\hline Non & 78,1 & 82,6 & 81,5 & 80,6 & 88,9 & 82,1 \\
\hline Total & 100,0 & 100,0 & 100,0 & 100,0 & 100,0 & 100,0 \\
\hline Effectif & 33 & 23 & 27 & 33 & 27 & 143 \\
\hline
\end{tabular}




\begin{tabular}{|c|c|c|c|c|c|c|}
\hline Motifs & & & & & & \\
\hline Manquement à la fidélité & 12,5 & 50,0 & 66,7 & 50,0 & 33,3 & 40,7 \\
\hline Non-assistance & 20,0 & 0,0 & 60,0 & 50,0 & 50,0 & 40,0 \\
\hline Non respect & 20,0 & 50,0 & 60,0 & 66,7 & 0,0 & 45,0 \\
\hline Non affection & 40,0 & 0,0 & 80,0 & 83,3 & 50,0 & 60,0 \\
\hline Absence de communauté & 60,0 & 0,0 & 60,0 & 50,0 & 50,0 & 50,0 \\
\hline Effectif & 7 & 4 & 5 & 6 & 3 & 26 \\
\hline
\end{tabular}

\begin{tabular}{lrrrrrr}
\hline $\begin{array}{l}\text { Avoir recours à une } \\
\text { structure ou à des } \\
\text { personnes pour régler un } \\
\text { problème }\end{array}$ & & & & & & \\
\hline Oui & 0,0 & 0,0 & 80,0 & 50,0 & 50,0 & 42,1 \\
\hline Non & 100,0 & 100,0 & 20,0 & 50,0 & 50,0 & 57,9 \\
\hline Total & 100,0 & 100,0 & 100,0 & 100,0 & 100,0 & 100,0 \\
\hline Effectif & 4 & 2 & 5 & 6 & 2 & 19 \\
\hline
\end{tabular}

\section{Responsabilités vis-à-vis des charges du ménage}

Au cours de l'enquête individuelle avec les adolescentes mariées, une série de questions leur a été posées concernant la personne qui assume les charges du ménage. Il ressort des résultats que le conjoint est principalement celui qui assume la responsabilité vis-à vis des charges du ménage pour 83, 6\% des cas. Dans une moindre mesure, les autres membres de la famille du conjoint ont la responsabilité des charges du ménage. Il s’agit essentiellement des cas où le couple vit avec les parents biologiques du mari.

Tableau 3.42 : Personne assumant principalement les charges du ménage (en \%)

\begin{tabular}{lrrrrrr}
\hline \begin{tabular}{l} 
Personne assumant $\begin{array}{l}\text { Région } \\
\text { principalement les } \\
\text { charges du ménage }\end{array}$ \\
\cline { 2 - 8 }
\end{tabular} & Centre-Sud & Est & Sahel & Centre-Est & $\begin{array}{r}\text { Centre- } \\
\text { Nord }\end{array}$ & Ensemble \\
\hline l'homme & 77,4 & 75,0 & 82,6 & 92,9 & 88,5 & 83,6 \\
\hline La femme & 3,2 & 0,0 & 0,0 & 0,0 & 0,0 & 0,8 \\
\hline $\begin{array}{l}\text { L'homme et } \\
\text { femme }\end{array}$ & 3,2 & 10,0 & 0,0 & 7,1 & 0,0 & 3,9 \\
\hline Autre parent & & & & & & \\
\hline Autre & 9,7 & 0,0 & 13,0 & 0,0 & 11,5 & 7,0 \\
\hline Total & 6,5 & 15,0 & 4,3 & 0,0 & 0,0 & 4,7 \\
\hline Effectif & 100,0 & 100,0 & 100,0 & 100,0 & 100,0 & 100,0 \\
\hline
\end{tabular}

\section{Centres de décision}

Les décisions concernant la vie du couple sont prises généralement par l'homme. Concernant la prise de décisions en matière de santé, l’homme décide pour $81,3 \%$ des cas et les deux (l'homme et la femme) décident pour 7,8\% des cas.

Pour ce qui concerne l'éducation des enfants, 58,6\%, 11,7\% et seulement 4,7\% des décisions sont prises respectivement par l'homme, les deux conjoints et par la femme. Cette tendance se maintient dans le cas de la prise de décision en matière de la santé des enfants. 
Tableau 3.43: Prise de décisions dans les couples (en \%)

\begin{tabular}{|c|c|c|c|c|c|c|}
\hline \multirow{2}{*}{$\begin{array}{l}\text { La personne qui } \\
\text { prend les décisions en } \\
\text { matière de santé }\end{array}$} & \multicolumn{6}{|c|}{ Région } \\
\hline & Centre-Sud & Est & Sahel & Centre-Est & Centre-Nord & Ensemble \\
\hline l'homme & 77,4 & 90,0 & 82,6 & 82,1 & 76,9 & 81,3 \\
\hline L'homme et la femme & 3,2 & 0,0 & & 17,9 & 15,4 & 7,8 \\
\hline Autre parent & 6,5 & 0,0 & 13,0 & 0,0 & 3,8 & 4,7 \\
\hline Autre & 12,9 & 10,0 & 4,3 & 0,0 & 3,8 & 6,3 \\
\hline Total & 100,0 & 100,0 & 100,0 & 100,0 & 100,0 & 100,0 \\
\hline Effectif & 33 & 23 & 27 & 33 & 27 & 143 \\
\hline \multicolumn{7}{|l|}{$\begin{array}{l}\text { La personne qui } \\
\text { prend les décisions en } \\
\text { matière d'éducation } \\
\text { des enfants }\end{array}$} \\
\hline l'homme & 64,5 & 35,0 & 52,2 & 75,0 & 57,7 & 58,6 \\
\hline La femme & 0,0 & 0,0 & 26,1 & 0,0 & 0,0 & 4,7 \\
\hline L'homme et la femme & 3,2 & 10,0 & 4,3 & 17,9 & 23,1 & 11,7 \\
\hline Autre parent & 9,7 & 5,0 & 13,0 & 0,0 & 11,5 & 7,8 \\
\hline Autre & 22,6 & 50,0 & 4,3 & 7,1 & 7,7 & 17,2 \\
\hline Total & 100,0 & 100,0 & 100,0 & 100,0 & 100,0 & 100,0 \\
\hline Effectif & 33 & 23 & 27 & 33 & 27 & 143 \\
\hline \multicolumn{7}{|l|}{$\begin{array}{l}\text { La personne qui } \\
\text { prend les décisions en } \\
\text { matière de santé des } \\
\text { enfants }\end{array}$} \\
\hline l'homme & 64,5 & 36,8 & 69,6 & 75,0 & 57,7 & 62,2 \\
\hline La femme & 3,2 & 0,0 & 4,3 & 0,0 & 7,7 & 3,1 \\
\hline L'homme et la femme & 6,5 & 15,8 & 8,7 & 14,3 & 15,4 & 11,8 \\
\hline Autre parent & 9,7 & 0,0 & 13,0 & 0,0 & 7,7 & 6,3 \\
\hline Autre & 16,1 & 47,4 & 4,3 & 10,7 & 11,5 & 16,5 \\
\hline Total & 100,0 & 100,0 & 100,0 & 100,0 & 100,0 & 100,0 \\
\hline Effectif & 33 & 23 & 27 & 33 & 27 & 143 \\
\hline
\end{tabular}

\section{Nature des conflits et mode de règlement}

A la question de savoir s'il y a parfois des conflits dans le couple, 30,7\% des adolescentes mariées ont répondu par l'affirmative. C'est dans la région du Centre-Est que plus de la moitié des adolescentes mariées ont déclaré l'existence de conflits dans leur couple. Cette proportion est respectivement de $15,4 \%$ et $21,7 \%$ dans les régions du Centre-Nord et du Sahel. Le type de conflits couramment cité concerne les discussions sur la gestion du ménage (62,5\%), les violences conjugales (20\%), les soins des enfants (17,5\%) et la mobilité (15\%).

En cas de conflits, les couples ont généralement recours aux parents du mari (52,5\%) et dans $25 \%$ des cas, ils ne font recours à personne. Les parents de l'adolescentes sont consultés dans une moindre mesure (10\%). Les structures telles que la justice (2\%) et les associations sont très peu sollicitées pour apporter des solutions aux couples en cas de conflit. 
Tableau 3.44 : Nature des conflits et mode de règlement (en \%)

\begin{tabular}{lrrrrrr}
\hline Nature des conflits & \multicolumn{7}{c}{ Région } \\
\cline { 2 - 7 } & Centre-Sud & Est & Sahel & Centre-Est & Centre-Nord & Ensemble \\
\hline Education des enfants & 0,0 & 0,0 & 0,0 & 20,0 & 0,0 & 7,5 \\
\hline Soin des enfants & 10,0 & 16,7 & 0,0 & 33,3 & 0,0 & 17,5 \\
\hline Gestion du ménage & 30,0 & 66,7 & 60,0 & 80,0 & 75,0 & 62,5 \\
\hline Violence conjugale & 0,0 & 0,0 & 20,0 & 46,7 & 0,0 & 20,0 \\
\hline Mobilité & 10,0 & 16,7 & 20,0 & 6,7 & 50,0 & 15,0 \\
\hline Emploi/commerce & 10,0 & 16,7 & 0,0 & 0,0 & 0,0 & 5,0 \\
\hline Autre & 50,0 & 0,0 & 0,0 & 6,7 & 25,0 & 17,5 \\
\hline Effectif & 10 & 6 & 5 & 15 & 4 & 40 \\
\hline & & & & & & \\
\hline Principal recours en cas & & & & & & \\
de conflits & 20,0 & 50,0 & 40,0 & 13,3 & 25,0 & 25,0 \\
\hline Personne & 40,0 & 50,0 & 60,0 & 60,0 & 50,0 & 52,5 \\
\hline Parents du mari & 20,0 & 0,0 & & 13,3 & & 10,0 \\
\hline parents de la femme & 0,0 & 0,0 & 0,0 & 13,3 & 25,0 & 7,5 \\
\hline Amis/voisins & 10,0 & 0,0 & 0,0 & 0,0 & 0,0 & 2,5 \\
\hline Justice & 10,0 & 0,0 & 0,0 & 0,0 & 0,0 & 2,5 \\
\hline Autre & 100,0 & 100,0 & 100,0 & 100,0 & 100,0 & 100,0 \\
\hline Total & 10 & 6 & 5 & 15 & 4 & 40 \\
\hline Effectif & & & &
\end{tabular}

\section{Recours en cas de besoins financiers}

En cas de besoins financiers dans les couples, 41,7\% des adolescentes font recours aux parents de leur mari, $40,2 \%$ préfèrent se résigner. Une proportion très faible de couples a recours à une banque ou aux tontines alors que $6,3 \%, 2,4 \%$ et $2,4 \%$ font recours respectivement à des amis/voisins, aux parents de l'adolescente et aux associations.

Tableau 3.45 : Recours en cas de besoins financiers dans les couples (en \%)

\begin{tabular}{lrrrrrr}
\hline $\begin{array}{l}\text { Recours principal des } \\
\text { couples en cas de besoins } \\
\text { financiers }\end{array}$ & Centre-Sud & Est & Sahel & Centre-Est & Centre-Nord & Ensemble \\
\hline Aucun recours & 51,6 & 50,0 & 18,2 & 32,1 & 46,2 & 40,2 \\
\hline Parents du mari & 25,8 & 35,0 & 68,2 & 53,6 & 30,8 & 41,7 \\
\hline parents de la femme & 3,2 & 5,0 & 0,0 & 0,0 & 3,8 & 2,4 \\
\hline Amis/voisins & 6,5 & 0,0 & 9,1 & 0,0 & 15,4 & 6,3 \\
\hline Associations & 6,5 & 0,0 & 4,5 & 0,0 & 0,0 & 2,4 \\
\hline Banques/Tontines & 0,0 & 0,0 & 0,0 & 7,1 & 0,0 & 1,6 \\
\hline Autre & 6,5 & 10,0 & 0,0 & 7,1 & 3,8 & 5,5 \\
\hline Total & 100,0 & 100,0 & 100,0 & 100,0 & 100,0 & 100,0 \\
\hline Effectif & 33 & 23 & 27 & 33 & 27 & 143 \\
\hline
\end{tabular}




\section{Thèmes de discussion dans les couples}

La discussion entre mari et femme n'est pas une chose fréquente dans les couples des adolescentes mariées. Néanmoins dans les couples où cela se fait, les thèmes de discussions les plus couramment abordés sont la vie du couple (72,7\%), les grossesses $(64,1 \%)$, les accouchements $(60,2 \%)$ et les mariages $(50 \%)$.

La planification familiale, la santé sexuelle et reproductive, l'éducation, la scolarisation des enfants et les opportunités en matière d'emploi sont abordés par les couples pour plus de $30 \%$ des cas.

Tableau 3.46 : Thèmes de discussion dans les couples (en \%)

\begin{tabular}{|c|c|c|c|c|c|c|}
\hline \multirow{2}{*}{$\begin{array}{l}\text { Discutez-vous dans le } \\
\text { couple des questions } \\
\text { relatives aux thèmes } \\
\text { suivants }\end{array}$} & \multicolumn{6}{|c|}{ Région } \\
\hline & Centre-Sud & Est & Sahel & Centre-Est & Centre-Nord & Ensemble \\
\hline $\begin{array}{l}\text { Planification Familiale } \\
\text { (PF) }\end{array}$ & 32,3 & 30,0 & 34,8 & 39,3 & 50,0 & 37,5 \\
\hline Accouchement & 51,6 & 55,0 & 39,1 & 78,6 & 73,1 & 60,2 \\
\hline Grossesse & 64,5 & 60,0 & 43,5 & 78,6 & 69,2 & 64,1 \\
\hline $\begin{array}{l}\text { Santé sexuelle et } \\
\text { reproductive }\end{array}$ & 41,9 & 25,0 & 30,4 & 39,3 & 57,7 & 39,8 \\
\hline $\begin{array}{l}\text { Education/Scolarisation des } \\
\text { enfants }\end{array}$ & 38,7 & 20,0 & 26,1 & 46,4 & 38,5 & 35,2 \\
\hline $\begin{array}{l}\text { Emploi/opportunités } \\
\text { d'affaires }\end{array}$ & 45,2 & 20,0 & 43,5 & 25,0 & 38,5 & 35,2 \\
\hline Mariage & 58,1 & 70,0 & 34,8 & 35,7 & 53,8 & 50,0 \\
\hline Vie du couple & 67,7 & 75,0 & 52,2 & 89,3 & 76,9 & 72,7 \\
\hline
\end{tabular}

\subsection{Connaissances, attitudes et pratiques en matière de la santé sexuelle et reproductive}

\subsubsection{Vie sexuelle de l'adolescente}

La majorité des adolescentes enquêtées (70,9 \% \%) ne sont pas sexuellement actives. Celles qui sont sexuellement actives ont eu leur premier rapport sexuel à l'âge de 15,8 ans en moyenne.

La moitié des adolescentes du Centre-Est ont eu leurs premiers rapports sexuels avant 16 ans. La moitié de celles du Centre-Sud, de l'Est et du Sahel ont eu ces rapports avant 17 ans et la moitié de celles du Centre-Nord avant 18 ans.

Tableau 3.47 : Caractéristiques de tendance centrale et de dispersion de l'âge aux premiers rapports sexuels (années)

\begin{tabular}{lrrrrrr}
\hline Caractéristiques & Centre-Sud & \multicolumn{1}{l}{ Est } & Sahel & Centre-Est & Centre-Nord & Ensemble \\
\hline Mode & 17,0 & 17,0 & 16,0 & 16,0 & 17,0 & 17,0 \\
\hline Médiane & 16,0 & 16,0 & 16,0 & 15,0 & 17,0 & 16,0 \\
\hline Moyenne & 16,1 & 16,2 & 15,4 & 15,1 & 16,2 & 15,8 \\
\hline Ecart type & 1,2 & 1,4 & 1,5 & 1,7 & 1,3 & 1,5 \\
\hline Effectif & 77 & 79 & 49 & 99 & 79 & 383 \\
\hline
\end{tabular}

Dans l'ensemble, on constate que plus de la moitié des adolescentes $(54,1 \%)$ n'ont pas utilisé un condom lors des premiers rapports sexuels. Le pourcentage d'adolescentes ayant déclaré avoir utilisé un condom lors des premiers rapports sexuels varie d'une région à l'autre. Il 
passe de 11\% (Centre-Est) à 62,1\% (Est). Il y a lieu de mettre l'accent sur la double protection au cours de l'intervention.

Graphique 3. 9: Utilisation du condom lors des premiers rapports sexuels

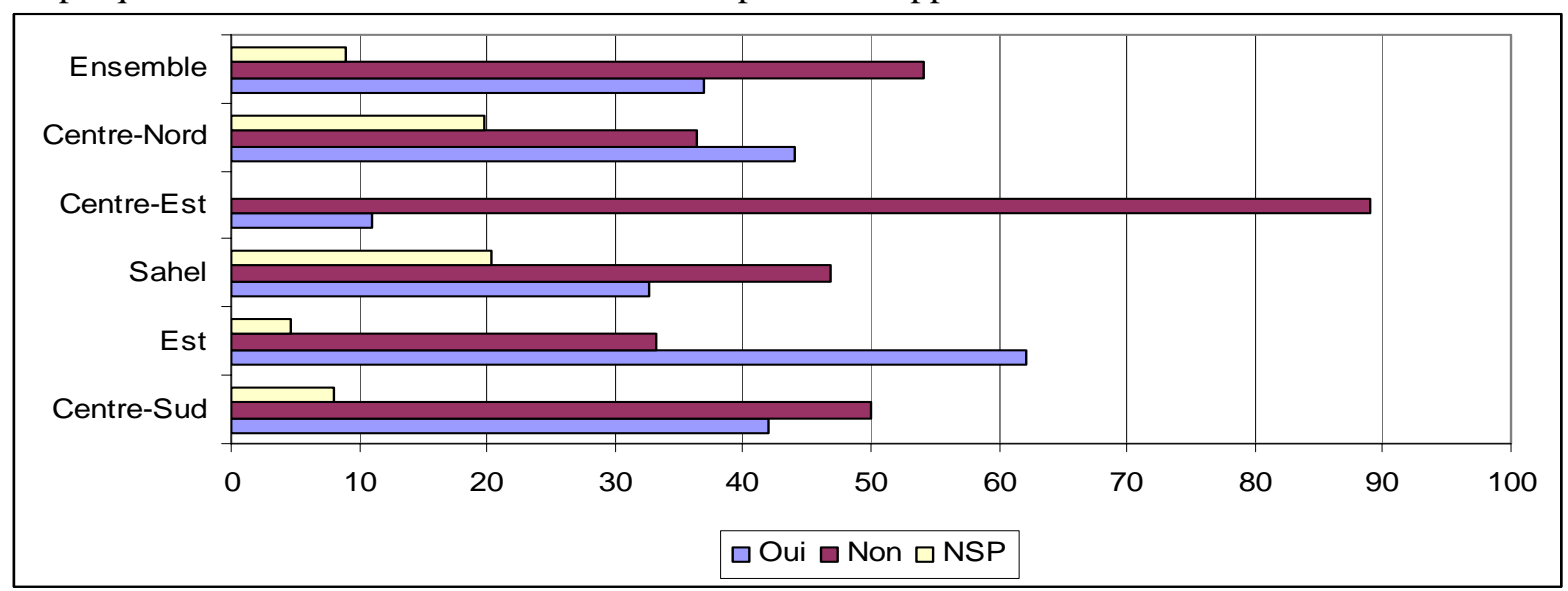

Effectifs : Centre-Sud (77), Est (79), Sahel (49), Centre-Est (99), Centre-Nord (79), Ensemble (383)

Moins de $40 \%$ des adolescentes déclarent avoir entendu parler de la période féconde. Par région, ce pourcentage varie de 35\% (Est) à 59,6\% (Sahel).

La période féconde est mal connue par les adolescentes dans l'ensemble des régions et plus particulièrement au Sahel, à l'Est et au Centre-Nord. Seulement 20,7\% des adolescentes ont déclaré que cette période se situe environ deux semaines avant le début des règles.

Tableau 3.48: Connaissance de la période féconde (en \%)

\begin{tabular}{|c|c|c|c|c|c|c|}
\hline \multirow{2}{*}{$\begin{array}{l}\text { Avoir entendu parler de la } \\
\text { période féconde }\end{array}$} & \multicolumn{6}{|c|}{ Région } \\
\hline & Centre-Sud & Est & Sahel & Centre-Est & Centre-Nord & Ensemble \\
\hline Oui & 36,5 & 35 & 59,6 & 37,2 & 37,8 & 38,6 \\
\hline Non & 63,5 & 65 & 40,4 & 62,8 & 62,2 & 61,4 \\
\hline Total & 100 & 100 & 100 & 100 & 100 & 100 \\
\hline Effectif & 295 & 288 & 116 & 361 & 260 & 1320 \\
\hline \multicolumn{7}{|l|}{$\begin{array}{l}\text { Connaissance de la période } \\
\text { féconde }\end{array}$} \\
\hline Juste avant le début des règles & 5,6 & 6 & 8,8 & 11,2 & 27,6 & 11,8 \\
\hline Pendant les règles & 3,7 & 12 & 5,9 & 2,2 & 14,3 & 7,3 \\
\hline Juste après les règles & 30,8 & 26 & 66,2 & 25,4 & 21,4 & 31,4 \\
\hline $\begin{array}{l}\text { Environ } 2 \text { semaines avant le } \\
\text { début des règles/mi-cycle }\end{array}$ & 23,4 & 16 & 5,9 & 32,1 & 17,3 & 20,7 \\
\hline N'importe quand du mois & 6,5 & 0 & 4,4 & 2,2 & 0 & 2,6 \\
\hline Autre & 1,9 & 1 & 0 & 0 & 5,1 & 1,6 \\
\hline Ne sait pas & 28 & 39 & 8,8 & 26,9 & 14,3 & 24,7 \\
\hline Total & 100 & 100 & 100 & 100 & 100 & 100 \\
\hline Effectif & 108 & 101 & 69 & 134 & 98 & 510 \\
\hline
\end{tabular}




\subsubsection{Connaissance et utilisation d'une méthode contraceptive}

\section{Connaissance de moyens pour éviter d'avoir une grossesse}

Dans l'ensemble, 42,7\% des répondantes déclarent connaître des moyens pour éviter une grossesse. Ce pourcentage varie par région passant de 37,2\% au Centre-Sud à 82,1\% au Sahel. Les moyens cités pour éviter une grossesse sont principalement le condom (73,1\%), la pilule (53\%), l'injection (36,3\%) et l'abstinence (31,8\%). Les autres moyens (le retrait, les spermicides, le DIU et le Norplant) ont été rarement cités. Cependant, les réponses varient par région. Ainsi, pour le condom, les pourcentages des réponses varient de 60,9\% au Sahel à 81,9\% au Centre-Nord ; en ce qui concerne la pilule, elles vont de $41 \%$ à l'Est à $62,3 \%$ au Centre-Est ; s'agissant de l'injection on enregistre des variations allant de $19 \%$ à l'Est à $59,8 \%$ au Sahel ; quant à l'abstinence, les variations dans les réponses vont de $20 \%$ au CentreNord à 52,2\% au Sahel.

Plus de 9 adolescentes sur 10 pensent que le planning familial est une bonne chose, mais elles n'arrivent pas à en donner les raisons. Une infime proportion d'adolescentes pense que c'est une mauvaise chose et la principale raison avancée est que le planning familial rend stérile.

Tableau 3.49 : Connaissance de moyens pour éviter -une grossesse (en \%)

\begin{tabular}{|c|c|c|c|c|c|c|}
\hline \multirow[t]{2}{*}{ Connaissance de moyens pour éviter d'avoir une grossesse } & \multicolumn{6}{|c|}{ Région } \\
\hline & Centre-Sud & Est & Sahel & Centre-Est & Centre-Nord & Ensemble \\
\hline Oui & 37,2 & 36,7 & 82,1 & 41,1 & 40,5 & 42,7 \\
\hline Non & 62,8 & 63,3 & 17,9 & 58,9 & 59,5 & $\overline{57,3}$ \\
\hline Total & 100,0 & 100,0 & 100,0 & 100,0 & 100,0 & 100,0 \\
\hline Effectif & 295 & 288 & 116 & 361 & 260 & 1320 \\
\hline \multicolumn{7}{|l|}{ Moyens pour éviter la grossesse } \\
\hline Pilule & 52,3 & 41,0 & 62,0 & 62,3 & 44,8 & 53,0 \\
\hline DIU & 0,9 & 2,9 & 1,1 & 0,7 & 2,9 & 1,6 \\
\hline Norplant & 21,1 & 20,0 & 16,3 & 11,6 & 21,0 & 17,6 \\
\hline Injection & 38,5 & 19,0 & 59,8 & 35,6 & 31,4 & 36,3 \\
\hline Condom & 67,9 & 73,3 & 60,9 & 78,1 & 81,9 & 73,1 \\
\hline Spermicide & 0,9 & 1,0 & 0,0 & 1,4 & 1,9 & 1,1 \\
\hline Ogino/calendrier & 0,9 & 12,4 & 23,9 & 0,7 & 2,9 & 7,2 \\
\hline Retrait & 0,0 & 0,0 & 0,0 & 2,1 & 1,0 & 0,7 \\
\hline Gris-gris ou méthode traditionnelle & 0,0 & 1,0 & 2,2 & 0,7 & 0,0 & 0,7 \\
\hline Abstinence & 31,2 & 21,9 & 52,2 & 34,9 & 20,0 & 31,8 \\
\hline Collier & 0,9 & 1,0 & 3,3 & 0,7 & 4,8 & 2,0 \\
\hline Effectif & 110 & 106 & 95 & 148 & 105 & 564 \\
\hline
\end{tabular}

\section{Utilisation de méthode contraceptive}

Au total, 43,3\% des adolescentes déclarent utiliser une méthode contraceptive, le plus fort pourcentage étant enregistré au Centre-Sud (58,7\%) et le plus faible au Sahel (28,3\%). Le condom apparaît comme étant la méthode la plus utilisée (66,5\% des réponses), variable de $53,8 \%$ au Sahel à 79,1\% au Centre-Est. Les lieux d'approvisionnement les plus cités sont par ordre d'importance la boutique, la pharmacie et le centre de santé. 


\section{Les canaux d'information sur le planning familial}

D’une manière générale, moins de la moitié des adolescentes $(47,7 \%)$ ont entendu parler du planning familial. Cela se confirme particulièrement dans les régions de l'Est, du Centre-Est et du Centre-Sud. Quant au Sahel et au Centre-Nord, plus de la moitié des adolescentes ont entendu parler du planning familial.

Les canaux d'informations citées par les adolescentes sont essentiellement la télévision (31,4\%), les amis (24,2\%), la radio (23,7\%), les réunions $(23,7 \%)$ et les parents $(10,5 \%)$. Cependant, rarement le mari a contribué à cela. Par région, les occasions sont variées et peuvent se résumer comme suit :

$\checkmark$ au Centre-Sud, c'est par les amis d'abord, ensuite lors d'une réunion, par la radio et enfin par la télévision ;

$\checkmark$ à l'Est, c'est d'abord par la télévision et ensuite lors d'une réunion ;

$\checkmark$ au Sahel, c'est par les amis d'abord, ensuite la télévision ;

$\checkmark$ au Centre-Est, c'est par la télévision d'abord, la radio, les amis et lors d'une réunion ;

$\checkmark$ au Centre-Nord, c'est par la radio d'abord, ensuite la télévision.

Tableau 3.50 : Sources d'information des adolescentes sur le planning familial (en \%)

\begin{tabular}{lrrrrrr}
\hline $\begin{array}{l}\text { Avoir déjà entendu } \\
\text { parler ou discuté du } \\
\text { planning familial ? }\end{array}$ & \multicolumn{7}{c}{ Région } \\
\cline { 2 - 8 } & Centre-Sud & \multicolumn{1}{c}{ Est } & Sahel & Centre-Est & Centre-Nord & Ensemble \\
\hline Non & 56,0 & 61,8 & 32,1 & 50,1 & 49,4 & 52,3 \\
\hline Oui & 44,0 & 38,2 & 67,9 & 49,9 & 50,6 & 47,7 \\
\hline Total & 100,0 & 100,0 & 100,0 & 100,0 & 100,0 & 100,0 \\
\hline Effectif & 295 & 288 & 116 & 361 & 260 & 1320 \\
\hline
\end{tabular}

A quelle occasion

?/avec qui ?

\begin{tabular}{lrrrrrr}
\hline Collègues de service & 0,0 & 0,0 & 0,0 & 3,4 & 0,8 & 1,1 \\
\hline Mari & 1,6 & 3,7 & 6,8 & 2,2 & 3,8 & 3,2 \\
\hline Parents & 16,3 & 2,8 & 4,1 & 18,5 & 3,8 & 10,5 \\
\hline Amis & 27,1 & 9,2 & 41,9 & 30,9 & 14,5 & 24,2 \\
\hline Lors d'une réunion & 24,8 & 12,8 & 17,6 & 25,3 & 15,3 & 20,0 \\
\hline Radio & 23,3 & 5,5 & 10,8 & 36,0 & 29,8 & 23,7 \\
\hline Télévision & 23,3 & 24,8 & 32,4 & 42,1 & 29,8 & 31,4 \\
\hline Association & 0,0 & 0,9 & 2,7 & 1,1 & 0,8 & 1,0 \\
\hline Effectif & 130 & 110 & 79 & 180 & 132 & 630 \\
\hline
\end{tabular}

\begin{tabular}{|c|c|c|c|c|c|c|}
\hline \multicolumn{7}{|l|}{ Opinion sur le PF } \\
\hline Une bonne chose & 91,5 & 82,6 & 84,0 & 96,0 & 85,9 & 89,2 \\
\hline Une mauvaise chose & 3,8 & 7,3 & 10,7 & 2,3 & 5,5 & 5,2 \\
\hline Pas nécessaire & 0,8 & 3,7 & 0,0 & 0,6 & 0,0 & $\overline{1,0}$ \\
\hline Pas d'opinion & 3,8 & 6,4 & 5,3 & 1,1 & 8,6 & 4,7 \\
\hline Total & 100,0 & 100,0 & 100,0 & 100,0 & 100,0 & 100,0 \\
\hline Effectif & 130 & 110 & 79 & 180 & 132 & 630 \\
\hline \multicolumn{7}{|c|}{$\begin{array}{lll}\begin{array}{l}\text { Raison } \\
\text { PF }\end{array} & \text { sur opinion } \\
\end{array}$} \\
\hline Rend stérile & 66,7 & 60,9 & 30,0 & 95,2 & 76,0 & 70,3 \\
\hline
\end{tabular}




\begin{tabular}{lrrrrrr}
\hline Rend maladie & 8,3 & 0,0 & 40,0 & 4,8 & 8,0 & 8,8 \\
\hline C'est Dieu qui donne & 0,0 & 21,7 & 30,0 & 0,0 & 12,0 & 12,1 \\
\hline A cause de la religion & 0,0 & 4,3 & 0,0 & 0,0 & 0,0 & 1,1 \\
\hline Autre & 25,0 & 13,0 & 0,0 & 0,0 & 4,0 & 7,7 \\
\hline Total & 100,0 & 100,0 & 100,0 & 100,0 & 100,0 & 100,0 \\
\hline Effectif & 6 & 12 & 8 & 5 & 7 & 39 \\
\hline
\end{tabular}

\subsubsection{Connaissances et attitudes par rapport au Sida}

La majorité des adolescentes (86,3\%) ont entendu parler du Sida. Par région, les pourcentages varient de 73,3\% (Centre-Sud) à 88,8\% (Centre-Nord). Ce pourcentage apparaît très bas au regard de l'importance des efforts faits par les acteurs pour sensibiliser la population.

Graphique 3.10 : Répartition des adolescentes selon qu’elles aient entendu parler ou non du VIH (en \%)

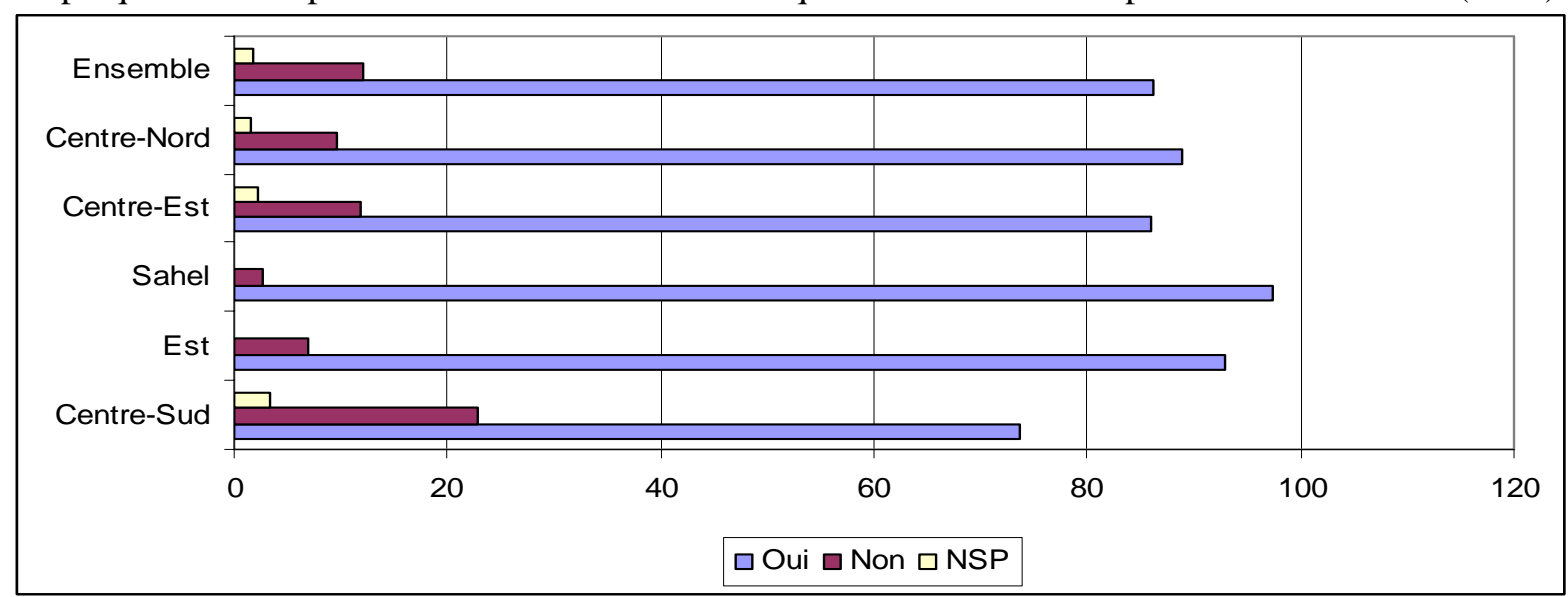

Effectifs : Centre-Sud (295), Est (288), Sahel (116), Centre-Est (361), Centre-Nord (260), Ensemble (1320)

Les relations sexuelles non protégées, le partage de seringue, la transfusion sanguine sont les voies de transmission du Sida les plus connues par les adolescentes de contracter le Sida.

Cependant certaines pensent encore que la piqûre d'un moustique ou autre insecte et le contact avec une personne infectée sont aussi des voies par lesquelles on peut contracter le Sida. Cependant, on constate que la transmission mère-enfant est très peu connue par les adolescentes $(7,4 \%)$.

Tableau 3.51 : Les moyens par lesquels un individu peut contracter le Sida (en \%)

\begin{tabular}{|c|c|c|c|c|c|c|}
\hline \multirow{2}{*}{$\begin{array}{l}\text { Mentionnez s'il vous plaît toutes les } \\
\text { manières dont selon vous, un } \\
\text { individu peut contracter le SIDA }\end{array}$} & \multicolumn{6}{|c|}{ Région } \\
\hline & Centre-Sud & Est & $\overline{\text { Sahel }}$ & Centre-Est & Centre-Nord & Ensemble \\
\hline Relations sexuelles non protégées & 78,7 & 61,0 & 85,3 & 71,3 & 73,0 & 72,0 \\
\hline Partage de seringue/objets souillés & 44,4 & 31,1 & 12,8 & 52,8 & 39,6 & 39,5 \\
\hline Transfusion sanguine & 24,1 & 4,9 & 10,1 & 29,0 & 23,0 & 19,3 \\
\hline Pendant la grossesse & 1,9 & 0,4 & 5,5 & 3,9 & 4,3 & 2,9 \\
\hline $\begin{array}{lllll}\text { De la mère } & \text { à l'enfant } & \text { durant } \\
\text { l'accouchement }\end{array}$ & 8,3 & 4,2 & 6,4 & 8,1 & 9,6 & 7,4 \\
\hline $\begin{array}{lcccc}\begin{array}{l}\text { Morsure } \\
\text { insectes }\end{array} & \text { de } & \text { moustique } & \text { ou } & \text { autre } \\
\end{array}$ & 2,8 & 0,8 & 0,0 & 0,0 & 0,9 & 0,9 \\
\hline Par le lait maternel & 1,9 & 1,1 & 1,8 & 1,3 & 0,9 & 1,3 \\
\hline Contact avec une personne infectée & 4,2 & 7,2 & 5,5 & 5,9 & 1,3 & 4,9 \\
\hline Autre & 0,9 & 14,7 & 43,1 & 2,0 & 3,0 & 9,0 \\
\hline NSP & 11,6 & 24,5 & 5,5 & 18,2 & 17,8 & $\overline{17,1}$ \\
\hline
\end{tabular}




\begin{tabular}{lrrrrrr}
\hline Effectif & 217 & 268 & 113 & 310 & 231 & 1139 \\
\hline & & & & & & \\
\hline $\begin{array}{l}\text { Pensez vous que le mariage protège } \\
\text { contre le SIDA ? }\end{array}$ & & & & & & \\
\hline Non & 38,0 & 48,9 & 51,4 & 34,2 & 27,4 & 38,7 \\
\hline Oui & 26,9 & 18,4 & 33,0 & 21,5 & 37,8 & 26,2 \\
\hline NSP & 35,2 & 32,7 & 15,6 & 44,3 & 34,8 & 35,1 \\
\hline Total & 100,0 & 100,0 & 100,0 & 100,0 & 100,0 & 100,0 \\
\hline Effectif & 217 & 268 & 113 & 310 & 231 & 1139 \\
\hline & & & & & & \\
\hline $\begin{array}{l}\text { Forme de mariage qui protège contre le } \\
\text { vIH/SIDA }\end{array}$ & & & & & & \\
\hline Aucune & 19,9 & 6,4 & 43,1 & 25,6 & 14,0 & 19,3 \\
\hline Monogamique & 2,3 & 0,4 & 18,3 & 0,3 & 4,4 & 3,3 \\
\hline Polygamique & 6,5 & 6,4 & 6,4 & 11,1 & 13,1 & 9,1 \\
\hline Monogamique et polygamique & 0,5 & 3,8 & 4,6 & 0,0 & 1,3 & 1,7 \\
\hline Lévirat/remariage & 0,5 & 2,3 & 2,8 & 7,5 & 2,2 & 3,4 \\
\hline Autre & 8,3 & 2,6 & 1,8 & 2,0 & 10,5 & 5,1 \\
\hline Ne sait pas & 62,0 & 78,2 & 22,9 & 53,4 & 54,6 & 58,2 \\
\hline Total & 100,0 & 100,0 & 100,0 & 100,0 & 100,0 & 100,0 \\
\hline Effectif & 79 & 53 & 38 & 78 & 98 & 346 \\
\hline & & & & & & \\
\hline
\end{tabular}

\begin{tabular}{lrrrrrr}
\hline $\begin{array}{l}\text { Une personne apparemment en bonne } \\
\text { santé peut -elle avoir le SIDA? }\end{array}$ \\
\hline Non & 28,2 & 15,8 & 14,7 & 8,5 & 24,3 & 17,8 \\
\hline Oui & 57,9 & 68,0 & 70,6 & 69,4 & 65,2 & 66,1 \\
\hline NSP & 13,9 & 16,2 & 14,7 & 22,1 & 10,4 & 16,0 \\
\hline Total & 100,0 & 100,0 & 100,0 & 100,0 & 100,0 & 100,0 \\
\hline Effectif & 295 & 288 & 116 & 361 & 260 & 1320 \\
\hline
\end{tabular}

\begin{tabular}{|c|c|c|c|c|c|c|}
\hline $\begin{array}{l}\text { Avez-vous fait le test de } \\
\text { VIH/SIDA? }\end{array}$ & & & & & & \\
\hline Oui & 15,7 & 13,2 & 14,7 & 8,6 & 15,2 & 13,0 \\
\hline Non & 84,3 & 86,8 & 85,3 & 91,4 & 84,8 & 87,0 \\
\hline Total & 100,0 & 100,0 & 100,0 & 100,0 & 100,0 & 100,0 \\
\hline Effectif & 217 & 268 & 113 & 310 & 231 & 1139 \\
\hline \multicolumn{7}{|l|}{ A quelle occasion? } \\
\hline Volontaire & 82,9 & 71,4 & 92,3 & 84,0 & 77,1 & 79,7 \\
\hline Imposé & 0,0 & 11,4 & 0,0 & 0,0 & 0,0 & 2,8 \\
\hline Lors d'une maladie & 2,9 & 5,7 & 0,0 & 0,0 & 5,7 & 3,5 \\
\hline Au cours d'une grossesse & 14,3 & 11,4 & 7,7 & 16,0 & 17,1 & 14,0 \\
\hline Total & 100,0 & 100,0 & 100,0 & 100,0 & 100,0 & $\overline{100,0}$ \\
\hline Effectif & 34 & 35 & 17 & 27 & 35 & 148 \\
\hline
\end{tabular}

Un peu plus d'un quart des adolescentes (26,2\%) pensent que le mariage peut contribuer à protéger contre le Sida. Ce pourcentage passe de 18,4\% (Est) à 37,8\% (Centre-Nord).

Près de deux tiers (66,1\%) des adolescentes pensent qu'une personne apparemment en bonne santé peut avoir le Sida. Les pourcentages varient par région, passant de 57,9\% (Centre-Sud) à $70,6 \%$ (Sahel). 
Une infime proportion d'adolescentes déclare avoir fait le test du VIH/Sida (13\%). Les pourcentages varient par région, allant de 8,6\% (Centre-Est) à 15,7\% (Centre-Sud).

C'est essentiellement de manière volontaire $(79,7 \%)$ ou au cours de la grossesse $(14 \%)$ que les adolescentes ont fait le test de dépistage du VIH.

L'enquête s'est également intéressée à la connaissance des adolescentes sur le lieu d'approvisionnement des préservatifs. Dans toutes les cinq régions, une forte proportion d'adolescentes $(90,9 \%)$ savent où se procurer des préservatifs. Les pourcentages vont de 82,9\% (Est) à 100\% (Centre-Est).

La boutique, la pharmacie et le marché sont les principaux lieux d'approvisionnement cités par les répondantes dans les régions du Centre-Sud et de l'Est. Celles du Sahel, du Centre-Est et du Centre-Nord ont plutôt mentionné la pharmacie, la boutique et le Centre de santé.

\subsubsection{Niveau de connaissance des fistules obstétricales, des visites prénatales et postnatales}

\section{Connaissance sur les grossesses à risque}

D’une manière générale, les adolescentes (59,4\%) déclarent ne pas connaitre les signes d'une grossesse à risque. Cela peut s'expliquer en partie par le faite qu'une grande partie des adolescentes interrogées n’a pas encore vécu l'expérience de la grossesse.

Le décès de la mère ou de l'enfant, l'âge de la mère (jeune ou âgée) et les difficultés liés à l'accouchement sont les conséquences les plus connues.

Tableau 3.52 : Connaissance sur les grossesses à risque

\begin{tabular}{|c|c|c|c|c|c|c|}
\hline \multirow[t]{2}{*}{ Signes d'une grossesse à risque } & \multicolumn{6}{|c|}{ Région } \\
\hline & Centre-Sud & Est & Sahel & Centre-Est & Centre-Nord & Ensemble \\
\hline Jeune âge/âge avancé de la mère & 24,9 & 13,6 & 59,8 & 26,3 & 27,1 & 26,2 \\
\hline Faible poids/poids élevé de la mère & $\overline{5,8}$ & 6,6 & 10,7 & 14,4 & 5,8 & 8,7 \\
\hline $\begin{array}{l}\text { Problèmes rencontrés lors des grossesses } \\
\text { antérieures }\end{array}$ & 2,7 & 0,0 & 1,8 & 4,2 & 2,3 & 2,4 \\
\hline Antécédents & 3,1 & 2,4 & 10,7 & 1,4 & 5,8 & 3,7 \\
\hline Absence de visites prénatales & 5,1 & 2,1 & 46,4 & 19,8 & 6,9 & 12,4 \\
\hline Ne sait pas & $\overline{62,0}$ & 74,1 & 10,7 & 61,1 & 59,1 & $\overline{59,4}$ \\
\hline Effectif & 295 & 288 & 116 & 361 & 260 & 1320 \\
\hline \multicolumn{7}{|l|}{$\begin{array}{l}\text { Quelles sont les conséquences d'une grossesse } \\
\text { précoce? }\end{array}$} \\
\hline Décès de la mère et/ou enfant & 30,6 & 24,1 & 55,9 & 50,7 & 36,3 & 37,8 \\
\hline Accouchement difficile/ césarienne & 51,2 & 41,3 & 82,9 & 38,7 & 35,3 & 45,2 \\
\hline Mère maladive & 7,6 & 9,4 & 11,7 & 7,0 & 6,2 & 7,9 \\
\hline Stérilité & 4,8 & 0,3 & 0,0 & 2,3 & 4,3 & 2,6 \\
\hline Fistule obstétricale & 0,0 & 0,7 & 0,9 & 0,9 & 0,4 & 0,5 \\
\hline Ne sait pas & 36,1 & 37,8 & 3,6 & 39,3 & 38,8 & 35,0 \\
\hline Effectif & 295 & 288 & 116 & 361 & 260 & 1320 \\
\hline
\end{tabular}




\section{Connaissances sur les fistules obstétricales}

La fistule obstétricale est la constitution d'une communication anormale entre la vessie et le vagin (fistule vésico-vaginale) ou entre la vessie et le rectum (fistule vesico-rectale). Elle survient d'ordinaire pendant un accouchement compliqué dû à l'étroitesse du bassin ou du rétrécissement de l'orifice vaginal et/ou vulvaire suite à l'excision.

Très peu d'adolescentes ont entendu parler de fistule obstétricale (moins de 10\%). L’écoulement permanent d'urine est le signe le plus connu de la fistule obstétricale.

Tableau 3.53 : Connaissances sur les fistules obstétricales

\begin{tabular}{lrrrrrr}
\hline \multirow{2}{*}{$\begin{array}{l}\text { Avoir attendu parler de fistule } \\
\text { obstétricale }\end{array}$} & \multicolumn{7}{c}{ Région } \\
\cline { 2 - 8 } Non & Centre-Sud & Est & Sahel & Centre-Est & Centre-Nord & Ensemble \\
\hline Oui & 92,1 & 94,4 & 82,0 & 87,3 & 94,5 & 90,9 \\
\hline Total & 7,9 & 5,6 & 18,0 & 12,7 & 5,5 & 9,1 \\
\hline Effectif & 100,0 & 100,0 & 100,0 & 100,0 & 100,0 & 100,0 \\
\hline & 295 & 288 & 116 & 361 & 260 & 1320 \\
\hline Signes des fistules obstétricales & & & & & & \\
\hline Ecoulement permanent d'urine & 43,5 & 56,3 & 20,0 & 72,3 & 25,0 & 49,2 \\
\hline Ecoulement permanent de selles & 4,3 & 0,0 & 5,0 & 51,1 & 0,0 & 20,8 \\
\hline $\begin{array}{l}\text { Ecoulement permanent d'urine et } \\
\text { de selles }\end{array}$ & 0,0 & 20,0 & 30,0 & 2,1 & 0,0 & 8,0 \\
\hline NSP & 30,4 & 25,0 & 45,0 & 19,1 & 35,0 & 28,6 \\
\hline Effectif & 23 & 16 & 21 & 46 & 14 & 120 \\
\hline
\end{tabular}

\section{Connaissances sur les visites pré et postnatales}

Le suivi de la grossesse est très important pour le bon déroulement de la grossesse et de l'accouchement. Il est recommandé au moins 4 visites prénatales chez la femme enceinte.

La majorité des adolescentes connaissent l'importance des visites prénatales pendant la grossesse. Cependant, elles sont peu nombreuses à savoir le nombre de visites. L'importance des visites postnatales est également bien connue par les adolescentes.

Tableau 3.54 : Connaissances des adolescentes sur les visites pré et postnatales

\begin{tabular}{|c|c|c|c|c|c|c|}
\hline \multirow{2}{*}{$\begin{array}{l}\text { Est ce qu'une femme doit } \\
\text { effectuer des visites pré- } \\
\text { natales }\end{array}$} & \multicolumn{6}{|c|}{ Région } \\
\hline & Centre-Sud & Est & Sahel & Centre-Est & Centre-Nord & Ensemble \\
\hline Non & 2,6 & 1,8 & 0,0 & 3,2 & 5,1 & 2,8 \\
\hline Oui & 96,3 & 98,2 & 99,1 & 89,1 & 91,1 & 94,0 \\
\hline NSP & 1,1 & 0,0 & 0,9 & 7,7 & 3,8 & 3,2 \\
\hline Total & 100,0 & 100,0 & 100,0 & 100,0 & 100,0 & 100,0 \\
\hline Effectif & 295 & 288 & 116 & 361 & 260 & 1320 \\
\hline \multicolumn{7}{|l|}{$\begin{array}{lll}\begin{array}{l}\text { Combien } \\
\text { prénatales }\end{array} & \text { de } & \text { visites } \\
\end{array}$} \\
\hline Une visite & 6,6 & 0,6 & 2,8 & 1,2 & 6,8 & 3,8 \\
\hline Deux visites & 10,0 & 2,8 & 0,0 & 4,6 & 14,8 & 7,3 \\
\hline Trois visites & 25,5 & 21,5 & 21,1 & 28,2 & 29,7 & 26,1 \\
\hline
\end{tabular}




\begin{tabular}{lrrrrrr}
\hline Quatre visites & 17,7 & 19,2 & 17,4 & 17,5 & 16,5 & 17,6 \\
\hline Plus de quatre visites & 34,3 & 55,4 & 58,7 & 30,7 & 30,1 & 38,1 \\
\hline NSP & 5,9 & 0,6 & 0,0 & 17,8 & 2,1 & 7,1 \\
\hline Total & 100,0 & 100,0 & 100,0 & 100,0 & 100,0 & 100,0 \\
\hline Effectif & 284 & 283 & 115 & 322 & 237 & 1241 \\
\hline
\end{tabular}

Est ce qu'une femme doit

effectuer des visites post

natales

\begin{tabular}{lrrrrrr}
\hline Oui & 96,3 & 98,2 & 99,1 & 89,1 & 91,1 & 94,0 \\
\hline Non & 2,6 & 1,8 & 0,0 & 3,2 & 5,1 & 2,8 \\
\hline NSP & 1,1 & 0,0 & 0,9 & 7,7 & 3,8 & 3,2 \\
\hline Total & 100,0 & 100,0 & 100,0 & 100,0 & 100,0 & 100,0 \\
\hline Effectif & 295 & 288 & 116 & 361 & 260 & 1320 \\
\hline
\end{tabular}

\subsection{Opportunités, attentes et besoins des adolescentes}

\subsubsection{Opportunités, attentes et besoins en éducation et en formation}

Très peu d'adolescentes ont reçu une formation professionnelle (7,4\%). Les pourcentages se situent entre 4,2\% pour le Centre-Est et 12,5\% pour le Centre-Sud.

Graphique 3.11 : Formation professionnelle des adolescentes enquêtées (en \%)

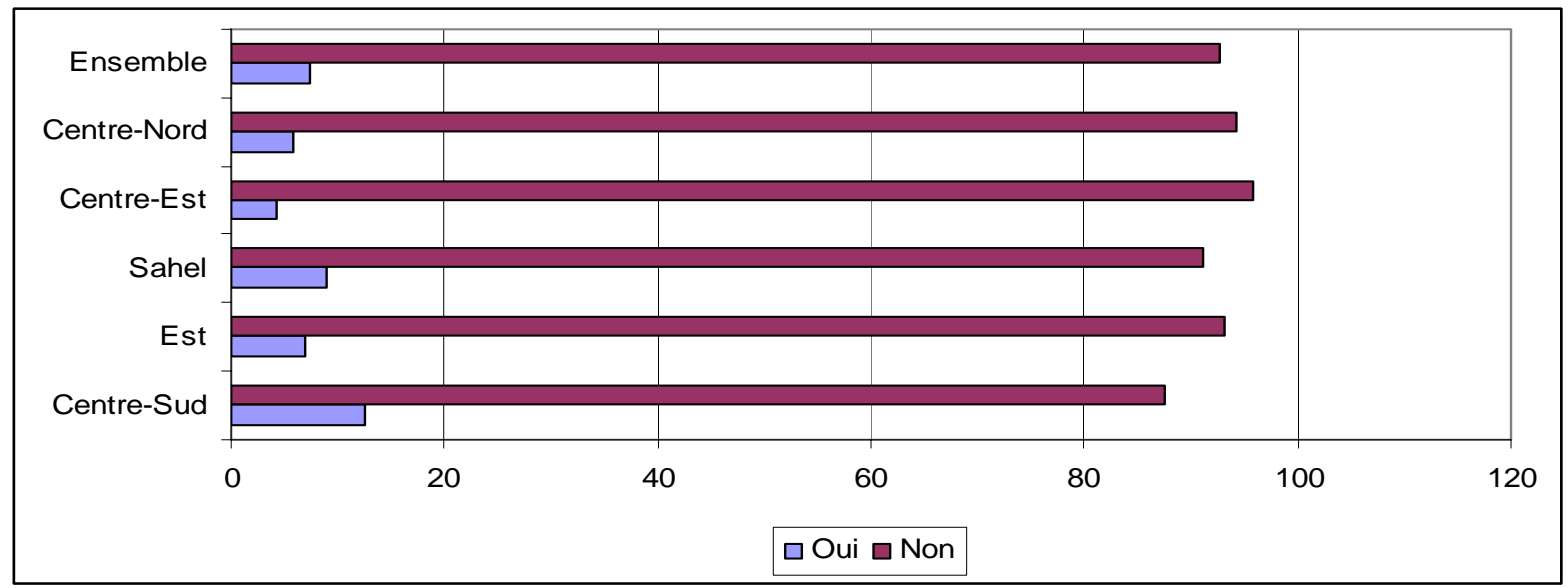

Effectifs : Centre-Sud (295), Est (288), Sahel (116), Centre-Est (361), Centre-Nord (260), Ensemble (1320)

Les types de formation professionnelle reçus par les adolescentes enquêtées varient d'une région à l'autre :

$\checkmark$ Au Centre-Sud, il s'agit de la gestion ou du commerce, du tissage/teinture/couture/broderie et de la transformation des produits ;

$\checkmark$ A l'Est, c'est essentiellement le tissage/teinture/couture/broderie ;

$\checkmark$ Au Sahel, on peut noter le tissage/teinture/couture/broderie, l'alphabétisation, le maraîchage et la coiffure ;

$\checkmark$ Au Centre-Est, il s'agit du tissage/teinture/couture/broderie ;

$\checkmark$ Au Centre-Nord, les activités professionnelles sont dominées par la gestion/commerce, le tissage/teinture/couture/broderie et la coiffure. 
Tableau 3.55 : Type de formation professionnelle reçus par les adolescentes enquêtées (en \%)

\begin{tabular}{lrrrrrr}
\hline $\begin{array}{l}\text { Type de formation } \\
\text { professionnelle reçu }\end{array}$ & \multicolumn{7}{c}{ Région } \\
\cline { 2 - 7 } & Centre-Sud & Est & Sahel & Centre-Est & Centre-Nord & Ensemble \\
\hline Transformation des produits & 16,2 & 0,0 & 0,0 & 7,1 & 0,0 & 7,5 \\
\hline Tissage/teinture/couture/broderie & 21,6 & 72,2 & 50,0 & 64,3 & 21,4 & 40,9 \\
\hline Maraîchage/pépinière & 2,7 & 0,0 & 10,0 & 0,0 & 0,0 & 2,2 \\
\hline Secrétariat & 2,7 & 5,6 & 0,0 & 0,0 & 0,0 & 2,2 \\
\hline Formation en animation & 0,0 & 0,0 & 0,0 & 0,0 & 7,1 & 1,1 \\
\hline Alphabétisation & 0,0 & 0,0 & 30,0 & 7,1 & 0,0 & 4,3 \\
\hline Gestion/commerce & 27,0 & 0,0 & 0,0 & 0,0 & 35,7 & 16,1 \\
\hline Maçonnerie/menuiserie & 0,0 & 5,6 & 0,0 & 0,0 & 0,0 & 1,1 \\
\hline Restauration & 5,4 & 0,0 & 0,0 & 7,1 & 7,1 & 4,3 \\
\hline Coiffure & 5,4 & 0,0 & 10,0 & 0,0 & 14,3 & 5,4 \\
\hline Formation en informatique & 2,7 & 0,0 & 0,0 & 7,1 & 7,1 & 3,2 \\
\hline Autre & 16,2 & 16,7 & 0,0 & 7,1 & 7,1 & 11,8 \\
\hline Total & 100,0 & 100,0 & 100,0 & 100,0 & 100,0 & 100,0 \\
\hline Effectif & 37 & 20 & 10 & 15 & 15 & 98 \\
\hline
\end{tabular}

Parmi celles qui n’ont pas fréquenté l'école formelle, très peu d'adolescentes sont alphabétisées, le pourcentage le plus bas étant enregistré au Centre-Est $(3,1 \%)$ et le plus élevé au Sahel (22\%).

Graphique 3.12 : Répartition des adolescentes enquêtées selon qu’elles savent lire ou écrire (en \%)

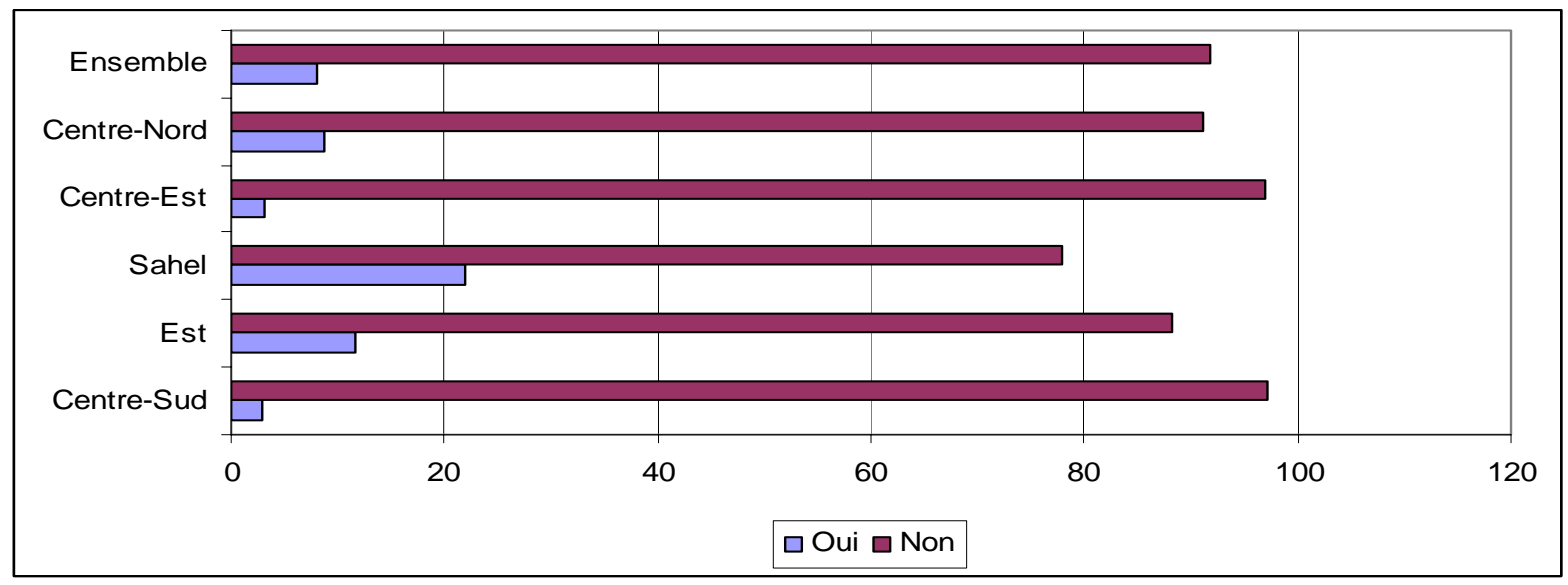

Effectifs : Centre-Sud (105), Est (77), Sahel (45), Centre-Est (66), Centre-Nord (56), Ensemble (349)

\section{Attentes et besoins de formation des adolescentes}

Selon les résultats, $80,8 \%$ des adolescentes qui ne fréquentent pas actuellement l'école souhaiteraient bénéficier d'une éducation ou d'une formation plus poussée. Parmi ceux-ci $45,8 \%$ d'entre elles souhaiteraient atteindre le supérieur, 46,4\% le niveau secondaire et seulement 2,4\% souhaiteraient s’arrêter au cycle primaire. 
Tableau 3.56 : Répartition des adolescentes selon le désir de poursuive une formation ou éducation plus poussée (en \%)

\begin{tabular}{lrrrrrr}
\hline $\begin{array}{l}\text { Souhaiteriez-vous bénéficier } \\
\text { d'une éducation ou d'une } \\
\text { formation plus poussée? }\end{array}$ & \multicolumn{7}{c}{ Région } \\
\cline { 2 - 8 } & Centre-Sud & Est & Sahel & Centre-Est & Centre-Nord & Ensemble \\
\hline Oui & 84,3 & 85,7 & 89,5 & 71,8 & 86,2 & 80,8 \\
\hline Non & 15,7 & 14,3 & 10,5 & 28,2 & 13,8 & 19,2 \\
\hline Total & 100,0 & 100,0 & 100,0 & 100,0 & 100,0 & 100,0 \\
\hline Effectif & 56 & 32 & 20 & 76 & 29 & 213 \\
\hline
\end{tabular}

Tableau 3.57 : Niveau d'instruction souhaité (en \%)

\begin{tabular}{lrrrrrr}
\hline Niveau d'instruction & \multicolumn{7}{c}{ Région } \\
\cline { 2 - 8 } & Centre-Sud & \multicolumn{1}{c}{ Est } & Sahel & Centre-Est & Centre-Nord & Ensemble \\
\hline Primaire & 4,3 & 4,0 & 0,0 & 1,9 & 0,0 & 2,4 \\
\hline Secondaire & 54,3 & 44,0 & 33,3 & 36,5 & 64,0 & 46,4 \\
\hline Supérieur & 28,3 & 44,0 & 66,7 & 61,5 & 32,0 & 45,8 \\
\hline Autre & 13,0 & 8,0 & 0,0 & 0,0 & 4,0 & 5,4 \\
\hline Total & 100,0 & 100,0 & 100,0 & 100,0 & 100,0 & 100,0 \\
\hline Effectif & 47 & 27 & 18 & 55 & 25 & 172 \\
\hline
\end{tabular}

\section{Besoins en formation professionnelle}

En matière de formation professionnelle, les adolescentes souhaiteraient se former dans les domaines tels que le tissage, teinture, couture et la broderie $(27,7 \%)$, dans la santé $(11,8 \%)$, dans la gestion ou le commerce $(10,3 \%)$ et dans une moindre mesure en informatique $(8,3 \%)$, en alphabétisation (6,4\%), en secrétariat (4\%) et en coiffure (4\%).

Par ailleurs, les résultats montrent que $89 \%$ des adolescentes seraient disponibles à participer dans des programmes visant à améliorer les domaines de l'éducation, de la santé et de l'emploi.

Tableau 3.58: Type de formation professionnelle souhaité par les adolescentes

\begin{tabular}{lrrrrrr}
\hline \multirow{2}{*}{$\begin{array}{l}\text { Type de formation } \\
\text { professionnelle souhaitée }\end{array}$} & \multicolumn{7}{c}{ Région } \\
\cline { 2 - 7 } & Centre-Sud & Est & Sahel & Centre-Est & Centre-Nord & Ensemble \\
\hline Transformation des produits & 3,7 & 0,3 & 0,9 & 0,6 & 1,9 & 1,5 \\
\hline Tissage/teinture/couture/broderie & 26,8 & 36,2 & 28,6 & 27,2 & 19,3 & 27,7 \\
\hline Maraîchage/pépinière & 1,0 & 0,0 & 0,0 & 0,6 & 0,4 & 0,5 \\
\hline Secrétariat & 3,1 & 1,4 & 0,9 & 5,1 & 7,7 & 4,0 \\
\hline Formation en animation & 0,3 & 0,0 & 0,9 & 1,1 & 0,4 & 0,5 \\
\hline Alphabétisation & 2,4 & 2,4 & 11,6 & 12,9 & 4,2 & 6,4 \\
\hline Gestion/commerce & 20,0 & 3,8 & 3,6 & 7,3 & 13,5 & 10,3 \\
\hline Gestion en dépôt pharmaceutique & 1,0 & 0,3 & 0,0 & 2,8 & 0,4 & 1,1 \\
\hline Formation en santé & 6,8 & 19,5 & 13,4 & 10,1 & 10,8 & 11,8 \\
\hline Maçonnerie/menuiserie & 0,0 & 2,1 & 0,0 & 0,0 & 0,0 & 0,5 \\
\hline Restauration & 4,7 & 1,4 & 0,0 & 6,2 & 1,9 & 3,4 \\
\hline Coiffure & 5,1 & 3,5 & 1,8 & 3,9 & 4,6 & 4,0 \\
\hline Formation en informatique & 1,7 & 2,1 & 2,7 & 3,7 & 3,5 & 2,8 \\
\hline Autre & 8,1 & 8,7 & 5,4 & 6,7 & 11,6 & 8,3 \\
\hline Ne sait pas & 15,3 & 18,1 & 29,5 & 11,8 & 19,7 & 17,0 \\
\hline Total & 100,0 & 100,0 & 100,0 & 100,0 & 100,0 & 100,0 \\
\hline Effectif & 295 & 288 & 116 & 361 & 260 & 1320 \\
\hline
\end{tabular}




\begin{tabular}{lrrrrrr}
\hline $\begin{array}{l}\text { Disponibilité des adolescentes } \\
\text { à participer a des } \\
\text { programmes de : }\end{array}$ & & & & & & \\
\hline Education & 87,1 & 94,1 & 75,9 & 92,7 & 88,1 & 89,4 \\
\hline Santé & 85,4 & 93,1 & 75,0 & 96,1 & 85,4 & 89,1 \\
\hline Emploi & 85,4 & 93,8 & 82,1 & 92,7 & 86,9 & 89,3 \\
\hline Effectif & 295 & 288 & 116 & 361 & 260 & 1320 \\
\hline
\end{tabular}

\section{Opportunités en éducation}

On note qu'en matière d'éducation les différentes localités concernées par l'enquête possèdent un certain nombre d'infrastructures. La quasi-totalité de celles-ci (97,9\%) possède une école primaire, $60,4 \%$ un lycée et $41,3 \%$ un CEG. Seulement $13,2 \%$ des localités ont un établissement d'enseignement technique. Au Centre-Sud (4,4\%) et au Centre-Est (8,8\%), très peu de localités ont un établissement d'enseignement technique.

Concernant la présence d'un centre d'alphabétisation, plus de la moitié (58\%) des adolescentes ont déclaré que leur localité possède un centre. Au Sahel, $91,1 \%$ des adolescentes enquêtées ont répondu par l'affirmative à la question si leur localité disposait d'un centre d'alphabétisation.

Cependant, la présence d'un centre de formation professionnelle dans les différentes localités n'est pas très fréquente. En effet, 32 \% des adolescentes ont déclaré que ce type de centre n'existe pas dans leur localité contre 36,4\% qui affirment son existence. Ceux qui déclarent ne rien savoir représentent 32\%. Les types de formations professionnelles dont disposent les différentes localités sont la coiffure ou la couture $(67,2 \%)$, la gestion ou le commerce $(15,3 \%)$ et dans une moindre mesure l'artisanat et la poterie $(9,1 \%)$ et la fabrication de produits alimentaires (4,7\%).

Tableau 3.59 : Les opportunités en matière d'éducation existant dans les régions

\begin{tabular}{lrrrrrr}
\hline $\begin{array}{l}\text { Les opportunités } \\
\text { d’éducation existant dans } \\
\text { les régions }\end{array}$ & \begin{tabular}{c} 
Centre-Sud \\
\cline { 2 - 7 }
\end{tabular} & Est & Sahel & Centre-Est & Centre-Nord & Ensemble \\
\hline Ecole maternelle & 40,0 & 51,7 & 43,8 & 41,1 & 49,2 & 45,0 \\
\hline Ecole primaire & 98,3 & 99,0 & 99,1 & 98,3 & 95,3 & 97,9 \\
\hline CEG & 42,4 & 45,5 & 25,0 & 43,9 & 39,1 & 41,3 \\
\hline Lycée & 40,3 & 71,2 & 76,8 & 59,2 & 65,9 & 60,4 \\
\hline Enseignement technique & 4,4 & 27,1 & 6,3 & 8,8 & 17,1 & 13,2 \\
\hline Autre & 1,0 & 2,1 & 0,9 & & 3,1 & 1,4 \\
\hline Effectif & 295 & 288 & 116 & 361 & 260 & 1320 \\
\hline
\end{tabular}

\subsubsection{Opportunités, attentes et besoins en santé de la reproduction}

\section{Attentes et besoins en santé sexuelle et reproductive}

On constate qu'en général la fréquentation des centres de santé par les adolescentes n’est pas une chose courante dans les localités. Au total, seulement 26,7\% des adolescentes enquêtées déclarent avoir déjà utilisé des services de santé de la reproduction, le plus faible pourcentage étant enregistré au Sahel (17\%) et le plus élevé au Centre-Est (32,2\%). 
Parmi les adolescentes ayant déjà utilisé des services de santé, 82\% d'entre elles déclarent avoir été satisfaites de ces services. Le degré de satisfaction varie selon la région, allant de 68,1\% au Centre-Est à 95,3\% à l’Est.

Les raisons de la non satisfaction évoquées par les enquêtées sont très variables par région.

Il ressort que l'éloignement du centre constitue un problème majeur à l'Est (100\%). Au Centre-Sud le temps d'attente (50\%), le mauvais accueil (40\%) et le manque de personnel (20\%) ont été cités comme raisons d'insatisfaction. Quant au Centre-Nord, l'éloignement du centre $(33,3 \%)$ et le mauvais accueil (33,3\%) sont les raisons avancées. Au Centre-Est, l'éloignement (26,9\%), les coûts élevés (26,9\%), le mauvais accueil $(15,4 \%)$ et le temps d'attente $(15,4 \%)$ ont été déclarés comme raisons d’insatisfaction.

Tableau 3.60 : Utilisation et niveau de satisfaction des centres de santé de la reproduction

\begin{tabular}{|c|c|c|c|c|c|c|}
\hline \multirow{2}{*}{$\begin{array}{l}\text { Utilisation de } \\
\text { centre de santé de } \\
\text { la reproduction }\end{array}$} & \multicolumn{6}{|c|}{ Région } \\
\hline & Centre-Sud & Est & Sahel & Centre-Est & Centre-Nord & Ensemble \\
\hline Oui & 27,4 & 22,4 & 17,0 & 32,2 & 27,4 & 26,7 \\
\hline Non & 72,6 & 77,6 & 83,0 & 67,8 & 72,6 & 73,3 \\
\hline Total & 100,0 & 100,0 & 100,0 & 100,0 & 100,0 & 100,0 \\
\hline Effectif & 295 & 288 & 116 & 361 & 260 & 1320 \\
\hline \multicolumn{7}{|l|}{$\begin{array}{l}\text { Degré de } \\
\text { satisfaction }\end{array}$} \\
\hline Très insatisfaite & 0,0 & 3,1 & 15,8 & 19,8 & 2,9 & 7,7 \\
\hline Insatisfaite & 12,7 & 0,0 & 5,3 & 9,9 & 5,7 & 7,4 \\
\hline Satisfaite & 81,0 & 95,3 & 78,9 & 68,1 & 90,0 & 82,0 \\
\hline Très satisfaite & 6,3 & 1,6 & 0,0 & 2,2 & 1,4 & 2,8 \\
\hline Total & 100,0 & 100,0 & 100,0 & 100,0 & 100,0 & 100,0 \\
\hline Effectif & 81 & 65 & 20 & 116 & 71 & 352 \\
\hline \multicolumn{7}{|l|}{$\begin{array}{l}\text { Pour quelles } \\
\text { raisons n'avez- } \\
\text { vous pas été } \\
\text { satisfaite? }\end{array}$} \\
\hline $\begin{array}{l}\text { Eloignement du } \\
\text { centre }\end{array}$ & 40,0 & 100,0 & 0,0 & 26,9 & 33,3 & 31,3 \\
\hline $\begin{array}{l}\text { Coûts élevés des } \\
\text { prestations }\end{array}$ & 10,0 & 0,0 & 0 & 26,9 & 11,1 & 18,8 \\
\hline Mauvais accueil & 40,0 & 0,0 & 0,0 & 15,4 & 33,3 & 22,9 \\
\hline $\begin{array}{l}\text { Temps d'attente } \\
\text { trop long }\end{array}$ & 50,0 & 0,0 & 0,0 & 15,4 & 11,1 & 20,8 \\
\hline $\begin{array}{l}\text { Manque de } \\
\text { personnel }\end{array}$ & 20,0 & 0,0 & 0,0 & 0,0 & 11,1 & 4,2 \\
\hline $\begin{array}{l}\text { Manque de } \\
\text { médicaments }\end{array}$ & 10,0 & 0,0 & 0,0 & 0,0 & 11,1 & 4,2 \\
\hline Autre raison & 20,0 & 0,0 & 0,0 & 3,8 & 0,0 & 6,3 \\
\hline Effectif & 37 & 0 & 6 & 36 & 15 & 98 \\
\hline
\end{tabular}

La fréquentation d'un centre de santé est conditionnée par un certain nombre de facteurs dont la qualité des services, la qualité des prestations et de l'accueil, etc. 
Dans l'ensemble, les besoins des adolescentes en santé sexuelle et reproductive ne sont pas pris en compte dans les 5 régions car moins de $50 \%$ des adolescentes sont satisfaites. Le Centre-Est $(42,3 \%)$ semble être la région où les besoins des adolescentes ont été le mieux pris en compte.

Les adolescentes enquêtées souhaitent que les améliorations suivantes soient faites en matière d'offre de services:

$\checkmark$ un centre de santé proche du domicile : 52,3\% des réponses au Centre-Est et 39,4\% au Centre-Nord ;

$\checkmark$ des coûts des médicaments abordables : 57,8\% des réponses au Centre-Est, 52,6\% au Sahel, 49,3\% au Centre-Nord et 42,2\% à l'Est ;

$\checkmark$ des médicaments disponibles : 41,3\% des réponses au Centre-Est, 32,9\% au CentreSud, $23,9 \%$ au Centre-Nord et $21,9 \%$ à l'Est ;

$\checkmark$ un personnel suffisant en quantité et en qualité : 34,2\% des réponses au Centre-Sud, 27,5\% au Centre-Est et 25,4\% au Centre-Nord ;

$\checkmark$ du personnel disponible : 53,2\% des réponses au Centre-Est et 21,1\% au Sahel.

Les besoins en services de santé exprimés par les adolescentes se profilent comme suit :

$\checkmark$ les besoins en planification familiale constituent 51,8\% des réponses au Centre-Est, 40,5\% au Sahel et 25,6\% au Centre-Sud ;

$\checkmark$ les besoins en centre d'écoute pour jeunes représentent 40,6\% des réponses au CentreEst ;

$\checkmark$ les besoins en services de vaccination comptent pour 41,5\% des réponses au CentreEst, 35,1\% au Sahel, 33,6\% au Centre-Nord, 26,6\% au Centre-Sud et 23,4\% à l'Est.

$\checkmark$ les services de counselling sont très peu demandés et concernent $4,2 \%$ des réponses pour l'ensemble des cinq régions ;

$\checkmark$ les besoins en centre de sensibilisation constituent 66,7\% des réponses du Sahel, $37,1 \%$ à l'Est et $22,1 \%$ au Centre-Est.

$\checkmark$ Les besoins d'informations en matière de santé sexuelle et reproductive, variables par région, peuvent se résumer aux aspects suivants :

o informations sur les IST : au Centre-Est (76,5\%), au Centre-Nord (39,0\%), à l'Est (35,4\%), Centre-Sud 34,2\% et au Sahel 27,9\% ;

o informations sur le Planning Familial : au Sahel (45\%), au Centre-Est (38,9\%), au Centre-Nord (24,7\%) et au Centre-Sud (22,9\%) ;

o informations sur les soins prénatals et postnatals: Sahel $(55,9 \%)$, Centre-Est $(33,6 \%)$;

o informations sur l'utilisation de la contraception : au Sahel $(44,1 \%)$ et au CentreEst (26,6\%).

Tableau 3.61 : Besoins des adolescentes en matière de santé de la reproduction

\begin{tabular}{|c|c|c|c|c|c|c|}
\hline \multirow{2}{*}{$\begin{array}{l}\text { Quel type d'amélioration } \\
\text { souhaiteriez-vous avoir? }\end{array}$} & \multicolumn{6}{|l|}{ Régions } \\
\hline & Centre-Sud & Est & Sahel & Centre-Est & Centre-Nord & Ensemble \\
\hline $\begin{array}{l}\text { Centre de santé proche du } \\
\text { domicile }\end{array}$ & 26,6 & 1,6 & 15,8 & 52,3 & 39,4 & 32,2 \\
\hline $\begin{array}{l}\text { Coûts des médicaments } \\
\text { abordables }\end{array}$ & 21,5 & 42,2 & 52,6 & 57,8 & 49,3 & 44,4 \\
\hline Médicaments disponibles & 32,9 & 21,9 & 10,5 & 41,3 & 23,9 & 30,4 \\
\hline $\begin{array}{l}\text { Personnel suffisant en } \\
\text { quantité et en qualité }\end{array}$ & 34,2 & 4,7 & 0,0 & 27,5 & 25,4 & 22,8 \\
\hline Personnel disponible & 11,4 & 10,9 & 21,1 & 53,2 & 11,3 & 25,1 \\
\hline Autre & 16,5 & 51,6 & 0,0 & 2,8 & 12,7 & 17,0 \\
\hline Effectif & 295 & 288 & 116 & 361 & 260 & 1320 \\
\hline
\end{tabular}




\begin{tabular}{|c|c|c|c|c|c|c|}
\hline $\begin{array}{l}\text { De quels services de santé } \\
\text { avez-vous besoin? }\end{array}$ & & & & & & \\
\hline Services de PF & 25,6 & 7,7 & 40,5 & 51,8 & 22,4 & 29,5 \\
\hline Centre d'écoute pour jeunes & 12,6 & 10,8 & 5,4 & 40,6 & 18,1 & 20,4 \\
\hline Vaccination & 26,6 & 23,4 & 35,1 & 41,5 & 33,6 & 32,1 \\
\hline Counselling & 2,7 & 0,7 & 0,9 & 8,4 & 5,4 & 4,2 \\
\hline Centre de sensibilisation & 13,7 & 37,1 & 66,7 & 22,1 & 18,1 & 26,5 \\
\hline Documentation/Posters & 0,3 & 1,4 & 0,0 & 2,8 & 0,0 & 1,1 \\
\hline Autre & 38,2 & 34,3 & 0,9 & 16,0 & 37,5 & 27,9 \\
\hline Effectif & 295 & 288 & 116 & 361 & 260 & 1320 \\
\hline \multicolumn{7}{|l|}{$\begin{array}{l}\text { Besoins en information en } \\
\text { Santé de la reproduction }\end{array}$} \\
\hline Sur les IST & 34,2 & 35,4 & 27,9 & 76,5 & 39,0 & 46,5 \\
\hline Sur le planning familial & 22,9 & 11,9 & 45,0 & 38,9 & 24,7 & 27,1 \\
\hline Sur les soins pré et postnatals & 10,9 & 21,4 & 55,9 & 33,6 & 18,1 & 24,7 \\
\hline $\begin{array}{l}\text { Sur l'utilisation de la } \\
\text { contraception }\end{array}$ & 15,7 & 8,1 & 44,1 & 26,6 & 13,9 & 19,1 \\
\hline Effectif & 295 & 288 & 116 & 361 & 260 & 1320 \\
\hline \multicolumn{7}{|l|}{$\begin{array}{l}\text { Satisfaction des besoins en } \\
\text { santé de la reproduction des } \\
\text { adolescentes mariées ou non }\end{array}$} \\
\hline Oui & 29,0 & 28,3 & 29,5 & 42,3 & 30,9 & 32,9 \\
\hline Non & 4,8 & 2,5 & 0,9 & 2,3 & 4,6 & 3,2 \\
\hline NSP & 66,2 & 69,3 & 69,6 & 55,5 & 64,5 & 63,9 \\
\hline Total & 100,0 & 100,0 & 100,0 & 100,0 & 100,0 & 100,0 \\
\hline Effectif & 295 & 288 & 116 & 361 & 260 & 1320 \\
\hline \multicolumn{7}{|l|}{ Si non, pourquoi ? } \\
\hline Coûts & 42,9 & 42,9 & 0,0 & 30,0 & 33,3 & 36,4 \\
\hline Pas de service spécifique & 14,3 & 0,0 & 100,0 & 0,0 & 8,3 & 9,1 \\
\hline Pas de personnel qualifié & 14,3 & 0,0 & 0,0 & 10,0 & 16,7 & 11,4 \\
\hline Autre & 28,6 & 57,1 & 0,0 & 60,0 & 41,7 & 43,2 \\
\hline Total & 100,0 & 100,0 & 100,0 & 100,0 & 100,0 & 100,0 \\
\hline Effectif & 14 & 7 & 1 & 8 & 12 & 42 \\
\hline
\end{tabular}

\subsubsection{Opportunités, attentes et besoins d'emploi et d'accès au crédit}

\section{Caractéristiques socioprofessionnelles des adolescentes}

La majorité des adolescentes enquêtées (56,1\%) sont des élèves. La proportion la plus élevée $(64,6 \%)$ des élèves est enregistrée dans la région du Centre-Nord alors que cette proportion est de 44,7\% dans le Centre-Sud.

Les travaux ménagers constituent la seconde activité principale qui occupe un grand nombre d'adolescentes (20,8\%). C'est dans la région du Centre-Sud (28,5\%) et au Sahel (27,7\%) que les pourcentages les plus élevées ont été observées, alors que le Centre-Est $(14,3 \%)$ et le Centre-Nord (15,4\%) ont enregistré les pourcentages les plus faibles.

L’agriculture $(4,7 \%)$, l'élevage $(1,1 \%)$, le commerce $(6,9 \%)$ et les autres (les citer) activités occupent moins les adolescentes. 
Tableau 3.62 : Activité principale des adolescentes (en \%)

\begin{tabular}{|c|c|c|c|c|c|c|}
\hline \multirow{2}{*}{$\begin{array}{l}\text { Quelle est votre activité } \\
\text { principale? }\end{array}$} & \multicolumn{6}{|c|}{ Région } \\
\hline & Centre-Sud & Est & Sahel & Centre-Est & Centre-Nord & Ensemble \\
\hline Aucun & 6,8 & 0,0 & 10,7 & 7,8 & 10,0 & 6,6 \\
\hline Travaux ménagers & 28,5 & 23,3 & 27,7 & 14,3 & 15,4 & 20,8 \\
\hline Coiffure/Couture/broderie & 0,7 & 2,4 & 4,5 & 0,8 & 1,2 & 1,5 \\
\hline $\begin{array}{l}\text { Transformation } \\
\text { produits }\end{array}$ & 0,3 & 0,0 & 0,9 & 0,0 & 0,0 & 0,2 \\
\hline Agriculture & 3,4 & 7,3 & 2,7 & 4,2 & 4,6 & 4,7 \\
\hline Elevage & 3,7 & 0,0 & 0,0 & 0,6 & 0,8 & 1,1 \\
\hline Artisanat & 0,0 & 0,3 & 0,0 & 0,8 & 0,0 & 0,3 \\
\hline Commerce & 8,8 & 3,8 & 7,1 & 10,4 & 3,1 & 6,9 \\
\hline Elève & 44,7 & 62,0 & 45,5 & 57,7 & 64,6 & $\overline{56,1}$ \\
\hline Ne sait pas & 0,3 & 0,0 & 0,0 & 1,7 & 0,0 & 0,5 \\
\hline Autre & 2,7 & 0,7 & 0,9 & 1,7 & 0,4 & 1,4 \\
\hline Total & 100,0 & 100,0 & 100,0 & 100,0 & 100,0 & 100,0 \\
\hline Effectif & 295 & 288 & 116 & 361 & 260 & 1320 \\
\hline
\end{tabular}

D’une manière générale, très peu d'adolescentes ont déjà effectué des travaux ménagers qui leur ont rapporté de l'argent en dehors de leur maison. Seulement 9,4\% d'entre elles ont déclaré avoir effectué des travaux domestiques rémunérés. Cette proportion est de 14,3\% dans le Centre-Sud et 5,4\% au Sahel.

Tableau 3.63 : Répartition des adolescentes selon l'exercice de travaux ménagers payants

\begin{tabular}{lrrrrrr}
\hline $\begin{array}{l}\text { Avoir } \begin{array}{c}\text { exercé } \\
\text { travaux } \\
\text { payant. }\end{array} \\
\text { domestiques }\end{array}$ & \multicolumn{7}{c}{ Région } \\
\cline { 2 - 8 } Oui & Centre-Sud & Est & Sahel & Centre-Est & Centre-Nord & Ensemble \\
\hline Non & 14,3 & 7,6 & 5,4 & 5,6 & 12,8 & 9,4 \\
\hline NSP & 85,7 & 92,4 & 94,6 & 94,1 & 85,7 & 90,2 \\
\hline Total & 0,0 & 0,0 & 0,0 & 0,3 & 1,6 & 0,4 \\
\hline Effectif & 100,0 & 100,0 & 100,0 & 100,0 & 100,0 & 100,0 \\
\hline
\end{tabular}

L'exercice d'une activité économique rémunérée par les adolescentes reste très faible dans toutes les régions. En effet, mis à part les travaux domestiques, 11,4\% des adolescentes déclarent avoir déjà effectué une activité génératrice de revenus. Il se situe à 29,5\% dans le Sahel, 14,6\% dans le Centre-Sud et seulement 4,9\% à l'Est.

La plupart des adolescentes ont débuté ces activités à un âge très jeune. En effet, 59,2\% des adolescentes enquêtées ont commencé une activité génératrice de revenus à moins de 15 ans. Cette proportion est de 70,5\% dans la région du Centre-Sud, 64,3\% à l'Est et atteint son plus bas niveau (45,5\%) dans le Sahel.

Tableau 3.64 : Activités économiques menées par les adolescentes (en \%)

\begin{tabular}{lrrrrrr}
\hline $\begin{array}{l}\text { Avez-vous déjà effectué } \\
\text { une activité } \\
\text { rémunératrice }\end{array}$ & Centre-Sud & Est & Sahel & Centre-Est & Centre-Nord & Ensemble \\
\cline { 2 - 8 } & 14,6 & 4,9 & 29,5 & 8,1 & 11,5 & 11,4 \\
\hline Oui & 85,4 & 95,1 & 70,5 & 91,9 & 88,5 & 88,6 \\
\hline Non & 100,0 & 100,0 & 100,0 & 100,0 & 100,0 & 100,0 \\
\hline Total & 294 & 288 & 112 & 356 & 260 & 1310 \\
\hline Effectif & & & & & & \\
\hline
\end{tabular}




\begin{tabular}{lrrrrrr}
\hline Age de début d'activité & & & & & & \\
\hline Moins de 15 ans & 70,5 & 64,3 & 45,5 & 58,6 & 56,3 & 59,2 \\
\hline $15-19$ ans & 29,5 & 35,7 & 54,5 & 41,4 & 43,8 & 40,8 \\
\hline Total & 100,0 & 100,0 & 100,0 & 100,0 & 100,0 & 100,0 \\
\hline Effectif & 44 & 14 & 33 & 29 & 32 & 152 \\
\hline
\end{tabular}

Les activités des adolescentes reposent essentiellement sur le commerce (37,3\%), les travaux domestiques $(9,3 \%)$ et la production alimentaire (4\%). Les autres types d'activités (la production alimentaire, la couture, la broderie) sont faiblement exercés par les adolescentes.

Dans le Centre-Sud, le commerce et la production alimentaire sont les activités qui occupent la majorité des adolescentes, dans le Sahel (48,5\%) et le Centre-Est $(51,7 \%)$ le commerce est la principale occupation des adolescentes enquêtées.

Le marché $(52,9 \%)$ et les domiciles des employés (16,3\%) sont les principaux lieux dans lesquels les adolescentes exercent leurs activités principales. Le pourcentage des adolescentes effectuant leurs activités au marché atteint 80\% dans la région de l'Est.

Tableau 3.65 : Type de travail exercé par les adolescentes (en \%)

\begin{tabular}{lrrrrrr}
\hline $\begin{array}{l}\text { Quel type de travail effectuez-vous } \\
\text { principalement à l'heure actuelle ? }\end{array}$ & Centre-Sud & Est & Sahel & Centre-Est & Centre-Nord & Ensemble \\
\hline Aucun & 18,6 & 64,3 & 27,3 & 17,2 & 51,6 & 31,3 \\
\hline Travaux domestiques & 14,0 & 7,1 & 0,0 & 10,3 & 12,9 & 9,3 \\
\hline $\begin{array}{l}\text { Production alimentaire, élevage, } \\
\text { bois, etc. destiné à la vente }\end{array}$ & 11,6 & 0,0 & 0,0 & 0,0 & 3,2 & 4,0 \\
\hline $\begin{array}{l}\text { Travaux dans d'autres exploitations } \\
\text { agricoles }\end{array}$ & 0,0 & 0,0 & 0,0 & 3,4 & 0,0 & 0,7 \\
\hline Emploi domestique payé & 0,0 & 0,0 & 0,0 & 6,9 & 3,2 & 2,0 \\
\hline Coiffure & 2,3 & 0,0 & 6,1 & 3,4 & 0,0 & 2,7 \\
\hline Couture/broderie & 2,3 & 0,0 & 9,1 & 0,0 & 0,0 & 2,7 \\
\hline Maçonnerie/menuiserie & & 0,0 & 3,0 & 0,0 & 0,0 & 0,7 \\
\hline Commerçant/vendeuse & 37,2 & 14,3 & 48,5 & 51,7 & 22,6 & 37,3 \\
\hline Animatrice/relais & 2,3 & & & & & 0,7 \\
\hline Autre & 11,6 & 14,3 & 6,1 & 6,9 & 6,5 & 8,7 \\
\hline Total & 100,0 & 100,0 & 100,0 & 100,0 & 100,0 & 100,0 \\
\hline Effectif & 43 & 14 & 33 & 29 & 31 & 150 \\
\hline & & & & & & \\
\hline Où effectuez-vous ce travail ? & & & & & & \\
\hline Dans la maison familiale/personnelle & 16,7 & 0,0 & 12,5 & 4,2 & 0,0 & 9,6 \\
\hline $\begin{array}{l}\text { Dans les } \\
\text { champs/familiaux/personnels }\end{array}$ & 2,8 & 0,0 & 0,0 & 0,0 & 6,7 & 1,9 \\
\hline $\begin{array}{l}\text { Dans des maisons appartenant à } \\
\text { d'autres }\end{array}$ & 11,1 & 20,0 & 8,3 & 25,0 & 26,7 & 16,3 \\
\hline Dans les champs des autres & 0,0 & & 0,0 & 4,2 & 0,0 & 1,0 \\
\hline Au marché & 50,0 & 80,0 & 54,2 & 50,0 & 53,3 & 52,9 \\
\hline Au magasin/boutique & 0,0 & 0,0 & 12,5 & 4,2 & 0,0 & 3,8 \\
\hline A l'atelier & 5,6 & 0,0 & 4,2 & 8,3 & 0,0 & 4,8 \\
\hline Autres & 13,9 & 0,0 & 8,3 & 4,2 & 13,3 & 9,6 \\
\hline Total & 100,0 & 100,0 & 100,0 & 100,0 & 100,0 & 100,0 \\
\hline Effectif & 36 & 5 & 24 & 24 & 15 & 104 \\
\hline & & & & & & \\
\hline
\end{tabular}




\section{Motivations des adolescentes pour le travail}

Plusieurs raisons poussent les adolescentes à travailler. Les raisons les plus couramment citées sont le besoin d'avoir de l'argent (64,3\%), la recherche de l'indépendance financière (26,2\%), pour ne pas être oisive (26,2\%) et la contribution au revenu familial (14,3\%). Ces raisons reviennent dans toutes les régions enquêtées. Un nombre important d'adolescentes travaillent dans l’intention de préparer leur trousseau de mariage.

Tableau 3.66 : Raisons évoquées par les adolescentes pour justifier leur travail (en \%)

\begin{tabular}{|c|c|c|c|c|c|c|}
\hline \multirow[t]{2}{*}{ Pourquoi travaillez-vous? } & \multicolumn{6}{|c|}{ Région } \\
\hline & $\begin{array}{r}\text { Centre- } \\
\text { Sud }\end{array}$ & Est & Sahel & Centre-Est & $\begin{array}{r}\text { Centre- } \\
\text { Nord }\end{array}$ & Ensemble \\
\hline Contribuer au revenu familial & 11,5 & 0,0 & 0,0 & 22,2 & 41,7 & 14,3 \\
\hline Economiser pour le mariage & 11,5 & 25,0 & 16,7 & 38,9 & 8,3 & 19,0 \\
\hline $\begin{array}{l}\begin{array}{l}\text { Economiser pour les frais de } \\
\text { scolarité }\end{array} \\
\end{array}$ & 11,5 & 0,0 & 4,2 & 5,6 & 8,3 & 7,1 \\
\hline $\begin{array}{l}\text { Avoir une activité/ne pas être } \\
\text { oisive }\end{array}$ & 7,7 & 25,0 & 41,7 & 22,2 & 41,7 & 26,2 \\
\hline Faire comme les autres & & 25,0 & 29,2 & 5,6 & & 10,7 \\
\hline Indépendance financière & 46,2 & 25,0 & 0,0 & 38,9 & 16,7 & 26,2 \\
\hline Pour avoir de l'argent & 46,2 & 50,0 & 75,0 & 83,3 & 58,3 & 64,3 \\
\hline $\begin{array}{l}\text { Prendre en charge ses parents } \\
\text { et/ou son ménage }\end{array}$ & 4,0 & 25,0 & 0,0 & 0,0 & 0,0 & 2,4 \\
\hline Autre & 4,0 & 50,0 & 8,7 & 0,0 & 8,3 & 7,3 \\
\hline Ne sait pas & 4,3 & 0,0 & 0,0 & 0,0 & 8,3 & 2,5 \\
\hline Effectif & 36 & 5 & 24 & 24 & 15 & 104 \\
\hline
\end{tabular}

La majorité des adolescentes actives exercent une activité permanente (54,4\%). Cependant $27,2 \%$ et $14,6 \%$ d'entre elles exercent respectivement une activité saisonnière ou journalière. Au Sahel, 75\% des adolescentes actives ont une activité permanente contre seulement 20,8\% qui ont une activité saisonnière. Dans le Centre-Sud, les proportions des adolescentes ayant une activité permanente et celles ayant une activité saisonnière s'également presque. Les activités permanentes priment dans les régions de l’Est et du Centre-Est.

La plupart des adolescentes exercent une activité indépendante (57,3\%) contre 14,6\% qui sont des salariées du privé et 15,5\% d'aides familiaux. La quasi-totalité de celles-ci (96,4\%) sont rémunérées en espèces contre seulement $2,4 \%$ et $1,2 \%$ respectivement en nature ou sous les deux formes.

Tableau 3. 67 : Nature de l'emploi exercé par les adolescentes (en \%)

\begin{tabular}{lrrrrrr}
\hline Nature de l'emploi & \multicolumn{7}{c}{ Région } \\
\cline { 2 - 8 } & Centre-Sud & \multicolumn{1}{c}{ Est } & Sahel & Centre-Est & Centre-Nord & Ensemble \\
\hline Permanent & 40,0 & 60,0 & 75,0 & 62,5 & 40,0 & 54,4 \\
\hline Saisonnier & 48,6 & 0,0 & 20,8 & 8,3 & 26,7 & 27,2 \\
\hline Journalier & 8,6 & 40,0 & 4,2 & 20,8 & 26,7 & 14,6 \\
\hline Autre & 2,9 & 0,0 & 0,0 & 8,3 & 6,7 & 3,9 \\
\hline Total & 100,0 & 100,0 & 100,0 & 100,0 & 100,0 & 100,0 \\
\hline Effectif & 35 & 5 & 24 & 24 & 15 & 103 \\
\hline & & & & & & \\
\hline $\begin{array}{l}\text { Situation dans cet } \\
\text { emploi }\end{array}$ & & & & & & \\
\hline \begin{tabular}{l} 
Indépendant \\
\hline
\end{tabular} & 54,3 & 60,0 & 66,7 & 50,0 & & 57,3 \\
\hline
\end{tabular}




\begin{tabular}{lrrrrrr}
\hline Salarié du privé & 11,4 & 20,0 & 16,7 & 12,5 & 20,0 & 14,6 \\
\hline Aide familial & 28,6 & 20,0 & 8,3 & 4,2 & 13,3 & 15,5 \\
\hline Apprenti/Stagiaire & 2,9 & 0,0 & 8,3 & 16,7 & 6,7 & 7,8 \\
\hline Bénévole & 2,9 & 0,0 & 0,0 & 0,0 & 0,0 & 1,0 \\
\hline Autre & 0,0 & 0,0 & 0,0 & 16,7 & 0,0 & 3,9 \\
\hline Total & 100,0 & 100,0 & 100,0 & 100,0 & 100,0 & 100,0 \\
\hline Effectif & 35 & 5 & 24 & 24 & 15 & 103 \\
\hline
\end{tabular}

\begin{tabular}{|c|c|c|c|c|c|c|}
\hline \multicolumn{7}{|l|}{$\begin{array}{l}\text { Le travail est-il } \\
\text { rémunéré? }\end{array}$} \\
\hline Oui & 69,4 & 100,0 & 91,7 & 87,5 & 73,3 & 80,8 \\
\hline Non & 30,6 & & 8,3 & 12,5 & 26,7 & 19,2 \\
\hline Total & 100,0 & 100,0 & 100,0 & 100,0 & 100,0 & 100,0 \\
\hline Effectif & 35 & 5 & 24 & 24 & 15 & 103 \\
\hline \multicolumn{7}{|l|}{$\begin{array}{l}\text { Nature de la } \\
\text { rémunération }\end{array}$} \\
\hline Espèce & 100,0 & 100,0 & 90,9 & 100,0 & 90,9 & 96,4 \\
\hline Nature & 0,0 & 0,0 & 4,5 & 0,0 & 9,1 & 2,4 \\
\hline $\begin{array}{lll}\text { Sous les } & \text { deux } \\
\text { formes } & & \\
\end{array}$ & 0,0 & 0,0 & 4,5 & 0,0 & 0,0 & 1,2 \\
\hline Total & 100,0 & 100,0 & 100,0 & 100,0 & 100,0 & 100,0 \\
\hline Effectif & 25 & 5 & 22 & 21 & 11 & 84 \\
\hline
\end{tabular}

\section{Gestion des revenus}

Une grande partie des adolescentes partagent leurs revenus avec les membres de leur famille. En effet, 87,9\% des adolescentes affirment partager leurs gains avec les parents. Ce résultat confirme les déclarations des adolescentes selon lesquelles, elles travaillent pour venir en aide à leurs familles au niveau des dépenses. Elles sont majoritaires (67,8\%) à décider elles-mêmes de l'utilisation de leurs gains contre 30,5\% qui déclarent que ce sont les parents qui décident.

Tableau 3.68: Gestion des gains reçus par les adolescentes (en \%)

\begin{tabular}{lrrrrrr}
\hline Utilisation de vos gains & \multicolumn{5}{c}{ Région } \\
\cline { 2 - 7 } & Centre-Sud & Est & Sahel & Centre-Est & Centre-Nord & Ensemble \\
\hline Garde tout moi-même & 20,0 & 40,0 & 40,9 & 47,6 & 20,0 & 33,7 \\
\hline En donne une partie & 72,0 & 40,0 & 59,1 & 52,4 & 80,0 & 62,7 \\
\hline Autre & 8,0 & 20,0 & 0,0 & 0,0 & 0,0 & 3,6 \\
\hline Total & 100,0 & 100,0 & 100,0 & 100,0 & 100,0 & 100,0 \\
\hline Effectif & 25 & 5 & 22 & 21 & 11 & 84 \\
\hline & & & & & & \\
\hline Avec qui partagez-vous \\
gains
\end{tabular}




\begin{tabular}{lrrrrrr}
\hline Moi-même & 75,0 & 66,7 & 62,5 & 58,3 & 75,0 & 67,8 \\
\hline Autre & 0,0 & 0,0 & 6,3 & 0,0 & 0,0 & 1,7 \\
\hline Total & 100,0 & 100,0 & 100,0 & 100,0 & 100,0 & 100,0 \\
\hline Effectif & 25 & 5 & 22 & 21 & 11 & 84 \\
\hline
\end{tabular}

Néanmoins, les adolescentes gardent sur elles une partie de leurs revenus. Cet argent est utilisé principalement pour l'achat des vêtements 79,5\%, pour la constitution du trousseau de mariage 22,9\% (dans le Sahel et le Centre-Est), comme fonds de commerce 18,1\% ou pour l'épargne (16,9\%) (à l'Est, Centre-Est et au Centre-Nord).

Tableau 3.69 : Utilisation de l'argent reçu par les adolescentes

\begin{tabular}{|c|c|c|c|c|c|c|}
\hline \multirow[t]{2}{*}{ Que faites-vous de l'argent que vous gagnez? } & \multicolumn{6}{|c|}{ Région } \\
\hline & Centre-Sud & Est & Sahel & Centre-Est & Centre-Nord & Ensemble \\
\hline Payer les frais de scolarité & 0,0 & 20,0 & 4,5 & 4,8 & 10,0 & 4,8 \\
\hline Prendre en charge ses parents et ou de son ménage & 0,0 & 0,0 & 22,7 & 14,3 & 30,0 & 13,3 \\
\hline Acheter de nouveaux vêtements & 68,0 & 80,0 & 90,9 & 85,7 & 70,0 & 79,5 \\
\hline Economiser pour le trousseau de mariage & 16,0 & 20,0 & 27,3 & 28,6 & 20,0 & 22,9 \\
\hline Fonds de commerce & 8,0 & 20,0 & 9,1 & 38,1 & 20,0 & 18,1 \\
\hline Epargne & 4,0 & 0,0 & 18,2 & 38,1 & 10,0 & 16,9 \\
\hline Achat de médicaments & 4,0 & 20,0 & 9,1 & 0,0 & 0,0 & 4,8 \\
\hline Autre & 24,0 & 20,0 & 18,2 & 4,8 & 20,0 & 16,9 \\
\hline Effectif & 25 & 5 & 22 & 21 & 11 & 84 \\
\hline \multicolumn{7}{|l|}{ Où gardez vous votre argent? } \\
\hline Caisse d'épargne & 0,0 & 20,0 & 4,5 & & 9,1 & 3,7 \\
\hline Tontine & 0,0 & 0,0 & 0,0 & 4,8 & 0,0 & 1,2 \\
\hline Avec un membre de la famille & 26,1 & 40,0 & 36,4 & 38,1 & 27,3 & 32,9 \\
\hline Par devers moi même & 52,2 & 20,0 & 54,5 & 57,1 & 36,4 & 50,0 \\
\hline Amie & 0,0 & 0,0 & 0,0 & 0,0 & 9,1 & 1,2 \\
\hline Autre & 21,7 & 20,0 & 4,5 & 0,0 & 18,2 & 11,0 \\
\hline Total & 100,0 & 100,0 & 100,0 & 100,0 & 100,0 & 100,0 \\
\hline Effectif & 25 & 5 & 22 & 21 & 11 & 84 \\
\hline
\end{tabular}

\section{Attentes et opportunités en matière d'emploi}

A la question de savoir dans quel secteur d'activité auriez-vous souhaité travailler, 32,6\% des adolescentes souhaitent exercer des travaux domestiques contre 23,3\% qui préfèrent l'agriculture/élevage et $21,9 \%$ d'entre elles souhaitent exercer une profession libérale. Les travaux domestiques sont le secteur d'activités dans lequel plus de la majorité des adolescentes 64,6\% souhaiteraient travailler; cette proportion est de 42,9\% dans le Sahel alors qu'il n'est que de $12,1 \%$ dans le Centre-Est. Ce choix est peut-être influencé par le faible niveau d'instruction des adolescentes.

Tableau 3.70 : Répartition des adolescentes selon le secteur d’activités souhaité travailler (en \%)

\begin{tabular}{lrrrrrr}
\hline Secteur d'activités souhaité travailler & \multicolumn{7}{c}{ Région } \\
\cline { 2 - 8 } & Centre-Sud & Est & Sahel & Centre-Est & Centre-Nord & Ensemble \\
\hline Aucun & 3,4 & 0,7 & 6,3 & 0,3 & 2,7 & 2,1 \\
\hline Agriculture/Elevage & 15,9 & 9,7 & 24,1 & 34,6 & 31,1 & 23,3 \\
\hline Administration & 9,2 & 2,8 & 3,6 & 2,8 & 7,8 & 5,3 \\
\hline Professions libérales & 30,5 & 6,3 & 17,0 & 27,8 & 23,3 & 21,9 \\
\hline
\end{tabular}




\begin{tabular}{lrrrrrr}
\hline Commerce & 2,4 & 5,6 & 0,0 & 16,6 & 1,9 & 6,7 \\
\hline Artisanat & 0,7 & 0,0 & 0,9 & 0,0 & 0,0 & 0,2 \\
\hline Travaux domestiques & 29,2 & 64,6 & 42,9 & 12,1 & 24,9 & 32,6 \\
\hline Autre & 8,8 & 10,4 & 5,4 & 5,9 & 8,2 & 8,0 \\
\hline Ne sait pas & 100,0 & 100,0 & 100,0 & 100,0 & 100,0 & 100,0 \\
\hline Effectif & 295 & 288 & 116 & 361 & 260 & 1320 \\
\hline
\end{tabular}

Pour 67,9\% des adolescentes, leur localité possède toutes les opportunités pour leur permettre d'atteindre leur souhait en matière d'emploi. Cette proportion est respectivement de $80 \%$ et $53,1 \%$ pour le Sahel et pour le Centre-Est.

Tableau 3.71 : Possibilité en emploi offerte par la localité (en \%)

\begin{tabular}{lrrrrrr}
\hline $\begin{array}{l}\text { Possibilité en } \\
\text { emploi offerte par } \\
\text { la localité }\end{array}$ & Centre-Sud & Est & Sahel & Centre-Est & Centre-Nord & Ensemble \\
\cline { 2 - 7 } Oui & 69,5 & 73,7 & 80,0 & 53,1 & 75,2 & 67,9 \\
\hline Non & 15,9 & 10,0 & 10,9 & 21,3 & 11,2 & 14,8 \\
\hline NSP & 14,6 & 16,3 & 9,1 & 25,6 & 13,6 & 17,3 \\
\hline Total & 100,0 & 100,0 & 100,0 & 100,0 & 100,0 & 100,0 \\
\hline Effectif & 295 & 288 & 116 & 361 & 260 & 1320 \\
\hline
\end{tabular}

\section{Accès au crédit}

L'appartenance à des groupes de tontine n'est pas une pratique couramment partagée chez les adolescentes. En effet, seulement 5\% des adolescentes ont déclaré qu'elles sont membres d'une tontine. Cependant, le Sahel se distingue des autres régions avec 15,2\% des adolescentes qui sont membres d'un groupe de tontine.

L'argent gagné à partir des tontines est destiné à l'achat des vêtements (52,5\%), au fonds de commerce (39\%), à la prise en charge de la famille (18\%) et également pour la constitution du trousseau de mariage $(16,4 \%)$.

Tableau 3.72 -Appartenance ou non à des groupes de tontine et utilisation des ressources (en \%)

\begin{tabular}{|c|c|c|c|c|c|c|}
\hline \multirow[t]{2}{*}{ Membre d'une tontine } & \multicolumn{6}{|c|}{ Région } \\
\hline & Centre-Sud & Est & Sahel & Centre-Est & Centre-Nord & Ensemble \\
\hline Oui & 3,4 & 3,8 & 15,2 & 4,8 & 4,2 & 5,0 \\
\hline Non & 96,6 & 96,2 & 84,8 & 95,2 & 95,8 & 95,0 \\
\hline Total & 100,0 & 100,0 & 100,0 & 100,0 & 100,0 & 100,0 \\
\hline Effectif & 295 & 288 & 116 & 361 & 260 & 1320 \\
\hline \multicolumn{7}{|l|}{$\begin{array}{l}\text { Qu'allez-vous faire avec } \\
\text { cette somme? }\end{array}$} \\
\hline Payer les frais de scolarité & 0,0 & 0,0 & 6,3 & 5,9 & 0,0 & 3,3 \\
\hline $\begin{array}{l}\text { Prendre en charge ses parents } \\
\text { et ou de son ménage }\end{array}$ & 10,0 & 0,0 & 18,8 & 29,4 & 20,0 & 18,0 \\
\hline $\begin{array}{l}\text { Acheter de nouveaux } \\
\text { vêtements }\end{array}$ & 20,0 & 50,0 & 68,8 & 64,7 & 40,0 & 52,5 \\
\hline $\begin{array}{l}\text { Economiser pour le trousseau } \\
\text { de mariage }\end{array}$ & 20,0 & 12,5 & 12,5 & 23,5 & 10,0 & 16,4 \\
\hline Fonds de commerce & 40,0 & 37,5 & 37,5 & 47,1 & 30,0 & 39,3 \\
\hline Epargne & & 12,5 & 12,5 & 23,5 & 20,0 & 14,8 \\
\hline Autre & 20,0 & 37,5 & 18,8 & 5,9 & 50,0 & 23,0 \\
\hline Effectif & 10 & 11 & 18 & 17 & 11 & 66 \\
\hline
\end{tabular}




\section{Accès aux micro-finances}

Les adolescentes n'ont pas l'habitude de contracter des crédits dans les institutions de microfinances bien que celles-ci soient présentes dans certaines localités. Cela est certainement dû aux manques d'informations sur les conditions d'accès.

Tableau 3.73 : Présence d'une institution de micro-finances dans la localité et accès au crédit des adolescentes (en \%)

\begin{tabular}{lrrrrrr}
\hline $\begin{array}{l}\text { Existence d'une } \\
\text { institution de micro- } \\
\text { finances dans votre } \\
\text { localité }\end{array}$ & \multicolumn{7}{l}{ Centre-Sud } & \multicolumn{1}{l}{ Est } & Sahel & Centre-Est & Centre-Nord & Ensemble \\
\hline Oui & 29,2 & 68,1 & 70,5 & 50,1 & 41,9 & 49,0 \\
\hline Non & 70,5 & 31,1 & 29,5 & 37,0 & 57,0 & 47,0 \\
\hline NSP & 0,3 & 0,8 & & 12,9 & 1,2 & 4,0 \\
\hline Total & 100,0 & 100,0 & 100,0 & 100,0 & 100,0 & 100,0 \\
\hline Effectif & 295 & 288 & 116 & 361 & 260 & 1320 \\
\hline
\end{tabular}

\begin{tabular}{lrrrrrr}
\hline $\begin{array}{l}\text { Accès } \\
\text { filles à les } \\
\text { finance }\end{array}$ & $\begin{array}{l}\text { jeunes } \\
\text { micro }\end{array}$ & & & & & \\
\\
\hline Oui & 27,9 & 35,2 & 38,0 & 61,9 & 25,9 & 40,5 \\
\hline Non & 36,0 & 41,5 & 35,4 & 9,7 & 33,3 & 29,6 \\
\hline NSP & 36,0 & 23,3 & 26,6 & 28,4 & 40,7 & 29,9 \\
\hline Total & 100,0 & 100,0 & 100,0 & 100,0 & 100,0 & 100,0 \\
\hline Effectif & 86 & 176 & 79 & 176 & 108 & 625 \\
\hline
\end{tabular}

\begin{tabular}{lrrrrrr}
\hline $\begin{array}{l}\text { Avoir eu recours à } \\
\text { ces microfinances }\end{array}$ & & & & & & \\
\hline Oui & 0,0 & 1,6 & 3,3 & 0,0 & 0,0 & 0,8 \\
\hline Non & 100,0 & 98,4 & 96,7 & 100,0 & 100,0 & 99,2 \\
\hline Total & 100,0 & 100,0 & 100,0 & 100,0 & 100,0 & 100,0 \\
\hline Effectif & 24 & 63 & 30 & 109 & 27 & 253 \\
\hline
\end{tabular}

\subsection{Les recours des adolescentes en cas de besoins d'information et de soutien}

$\mathrm{Au}$ cours de l'enquête, l'une des préoccupations était de savoir qui sont les personnes ressources auprès desquelles les adolescentes s'informent concernant les sujets tels les fiançailles, le mariage, la vie de famille, les problèmes de santé, les opportunités d'emploi, de scolarisation et sur la gestion des conflits. Les résultats de l'étude font ressortir:

\section{Fiançailles, mariage ou vie de famille}

Pour ce qui concerne les fiançailles, le mariage ou la vie de famille, la mère (22\%) et les amies $(17,4 \%)$ sont les deux principales personnes ressources auprès desquelles les adolescentes s'informent sur ces sujets. 14,6 d'entre elles affirment s'informer auprès de leur père.

\section{Questions de santé}

Le père $(28,7 \%)$ et la mère $(21,1 \%)$ sont les principales personnes chez qui les adolescentes s'informent sur les questions de santé. Les amies et les maris sont des personnes ressources respectivement pour 7,6\% et 5,4\% des cas. Environ 14,5\% des adolescentes n'en parlent à personne. 


\section{Opportunités d'emploi}

Concernant les opportunités d'emploi, le père est la principale personne chez qui les adolescentes s'informent (22,4\%). La mère $(9,1 \%)$ et les frères $(6,5 \%)$ viennent respectivement en seconde et troisième position. Une grande proportion des adolescentes $(40,3 \%)$ ne consulte personne pour ces questions.

\section{Opportunités de scolarisation}

Pour les questions concernant la scolarisation, 23\% des adolescentes s'informent auprès de leurs mères, $13,8 \%$ et 10,7\% s’informent respectivement chez leur père et chez leurs amies.

\section{Gestion de conflits}

La mère (23\%), le père $(13,8 \%)$ et les amies $(10,7 \%)$ sont les personnes à qui les adolescentes se réfèrent pour la gestion des conflits.

\section{Appartenance à un groupe ou association}

L'appartenance à un groupe ou à une association est relativement faible chez les adolescentes (5,7\%). Cette proportion varie entre 3,1\% au Centre-Est et 8,9\% dans le Sahel.

Pour les adolescentes appartenant à un groupe ou à une association, les plus nombreuses sont membres d'un groupe de tontine (29,3\%) et d'un groupement ou association $(21,3 \%)$.

Les raisons qui les motivent à appartenir à l'un ou l'autre groupe sont l'élargissement des connaissances $(59,5 \%)$, la recherche d'un soutien financier $(43,2 \%)$, le soutien émotionnel $(28,4 \%)$ et la recherche des opportunités $(13,5 \%)$.

Tableau 3.74: Personnes ressources pour appartenir à une association (en \%)

\begin{tabular}{|c|c|c|c|c|c|c|}
\hline \multirow{2}{*}{$\begin{array}{l}\text { Appartenance à une } \\
\text { association }\end{array}$} & \multicolumn{6}{|c|}{ Région } \\
\hline & Centre-Sud & Est & Sahel & Centre-Est & Centre-Nord & Ensemble \\
\hline Oui & 6,5 & 5,6 & 8,9 & 3,1 & 7,3 & 5,7 \\
\hline Non & 93,5 & 94,4 & 91,1 & 96,9 & 92,7 & 94,3 \\
\hline Total & 100,0 & 100,0 & 100,0 & 100,0 & 100,0 & 100,0 \\
\hline Effectif & 294 & 288 & 112 & 357 & 260 & 1311 \\
\hline \multicolumn{7}{|l|}{ Type d'association } \\
\hline ONG & 0,0 & 0,0 & 0,0 & 0,0 & 0,0 & 0,0 \\
\hline Club sportif & 0,0 & 12,5 & 10,0 & 27,3 & 10,5 & 10,7 \\
\hline Groupement/Association & 10,5 & 6,3 & 60,0 & 0,0 & 36,8 & 21,3 \\
\hline Tontine & 15,8 & 31,3 & 30,0 & 63,6 & 21,1 & 29,3 \\
\hline Autre & 73,7 & 43,8 & 0,0 & 9,1 & 36,8 & 38,7 \\
\hline Effectif & 19 & 16 & 10 & 11 & 19 & 75 \\
\hline \multicolumn{7}{|l|}{$\begin{array}{l}\text { Bénéfices tirés de la } \\
\text { fréquentation de ces } \\
\text { groupes }\end{array}$} \\
\hline $\begin{array}{l}\text { Elargissement des } \\
\text { connaissances }\end{array}$ & 77,8 & 50,0 & 70,0 & 27,3 & 63,2 & 59,5 \\
\hline $\begin{array}{l}\text { Elargissement des } \\
\text { opportunités }\end{array}$ & 22,2 & 6,3 & 20,0 & 9,1 & 10,5 & 13,5 \\
\hline Soutien émotionnel & 38,9 & 25,0 & 30,0 & 0,0 & 36,8 & 28,4 \\
\hline Soutien financier & 44,4 & 31,3 & 40,0 & 54,5 & 47,4 & 43,2 \\
\hline Autre & 0,0 & 18,8 & 10,0 & 36,4 & 15,8 & 14,9 \\
\hline Effectif & 19 & 16 & 10 & 11 & 19 & 75 \\
\hline
\end{tabular}




\subsection{Mobilité des adolescentes}

La mobilité des adolescentes permet de renseigner sur les créneaux propices pour les formations/apprentissage ou séances de sensibilisation en faveur de ces dernières. Il est donc important de savoir là où elles peuvent se rendre facilement et là où elles ont des difficultés d'accès afin de pouvoir anticiper toute entorse au bon déroulement des activités.

Il ressort que la grande majorité des adolescentes se sont déjà déplacées (98,9\%) dans différents endroits publics de leur localité. Quelle que soit la destination, elles ont fortement besoin d'une permission pour se déplacer. Cependant, il convient de noter qu'elles ont plus de possibilité de se rendre dans certains lieux que dans d'autres comme en témoignent les différents résultats du tableau.

Concernant la mobilité des adolescentes au cours des trois derniers mois ayant précédé l'enquête, il ressort que la majorité des adolescentes se sont rendues dans les lieux où elles avaient déclaré qu'elles avaient plus de possibilité de s'y rendre, et dans la plupart des cas elles y sont allées seules. Une faible proportion de celles ayant moins de possibilité s'est rendue dans les lieux dont l’accès ne leur était pas d’emblée autorisée.

Les lieux de loisirs, les débits de boisson, les centres communautaires/place publique, les lieux d'événements sportifs et les déplacements en dehors du village/ville sont des situations qui requièrent particulièrement une permission et un accompagnement. L'ami reste le principal accompagnant.

Tableau 75: Informations sur la mobilité des adolescentes

\begin{tabular}{|c|c|c|c|c|c|}
\hline Destination & $\begin{array}{l}\text { Possibilité } \\
\text { de vous } \\
\text { rendre dans } \\
\text { ces lieux } \\
\quad(\%)\end{array}$ & $\begin{array}{l}\text { Besoin } \\
\text { d'une } \\
\text { permis- } \\
\text { sion } \\
\quad(\%)\end{array}$ & $\begin{array}{l}\text { Adolescentes } \\
\text { qui se sont } \\
\text { rendues } \\
\text { dans ces lieux } \\
\text { au } \\
\text { cours des } 3 \\
\text { derniers } \\
\text { mois (\%) } \\
\end{array}$ & $\begin{array}{c}\text { Accom- } \\
\text { pagnée } \\
(\%)\end{array}$ & $\begin{array}{l}\text { Principal (e) } \\
\text { accompa- } \\
\text { gnant(e) } \\
\qquad(\%)\end{array}$ \\
\hline a)Marché de la localité & 97,1 & 61,8 & 91,7 & 22,8 & Ami $(49,1)$ \\
\hline b)Centre de santé de la localité pour vos propres besoins & 73,4 & 71,1 & 38,1 & 31,9 & Ami $(24,2)$ \\
\hline $\begin{array}{l}\text { c)Centre de santé de la localité pour les besoins d'autres } \\
\text { membres de la famille }\end{array}$ & 59 & 73,7 & 27,7 & 37,3 & Ami $(24,4)$ \\
\hline d)Domicile des voisins & 74,2 & 54,9 & 86,1 & 10,4 & Ami $(58,4)$ \\
\hline e)Domiciles des amis/amies & 93 & 58,6 & 88,8 & 13 & Ami $(66,9)$ \\
\hline f)Domiciles parentaux & 83,5 & 67,6 & 60,7 & 19,2 & Ami $(25,5)$ \\
\hline g)Mariage ou cérémonies religieuses & 76,8 & 76,6 & 38,4 & 42,5 & Ami $(54,1)$ \\
\hline h)La coiffeuse & 81,4 & 70,8 & 66,9 & 25,1 & Ami $(47,6)$ \\
\hline i)Le tailleur & 82,3 & 73,1 & 58,4 & 34,6 & Ami $(43,3)$ \\
\hline $\begin{array}{l}\text { j)Loisirs(cinéma/concerts/kermesse ;théâtre/centre } \\
\text { culturel ;danse, vidéo-club) }\end{array}$ & $* 45,9$ & 80,6 & $* * 15,6$ & $* * * 59,9$ & Ami $(68,6)$ \\
\hline k)Centres communautaires ;place publique & $* 43,3$ & 82,2 & $* * 12,5$ & $* * * 53,8$ & Ami $(68,3)$ \\
\hline l)Evénement sportif & $* 38$ & 76,8 & $* * 11,7$ & $* * * 53,4$ & Ami (60) \\
\hline m)Visite de condoléances/funérailles & $* 32,6$ & 74 & $* * 10,5$ & 48,1 & Ami $(38,3)$ \\
\hline n)Centre administratif & $* 33,1$ & 77,6 & $* * 8$ & 36,1 & Ami $(37,1)$ \\
\hline o)En dehors du village/ville & 51,7 & 92,1 & $* * 20,8$ & $* * * 50$ & Ami $(22,5)$ \\
\hline p)Participer à une réunion de groupe féminin & $* 32$ & 75,7 & $* * 5,5$ & 33,3 & Ami $(71,1)$ \\
\hline $\begin{array}{l}\text { q)Participer à une éducation } \\
\text { formelle/alphabétisation }\end{array}$ & $* 23$ & 80,3 & $* * 2,5$ & 26,7 & Ami $(77,8)$ \\
\hline r)Débit de boisson & $* 8,9$ & 68,1 & $* * 4,2$ & $* * * 56$ & Ami $(69,2)$ \\
\hline Effectif & 1320 & 1320 & 1320 & 1320 & 1320 \\
\hline
\end{tabular}




\subsection{Relation entre le mariage précoce et certaines caractéristiques sociodémographiques et économiques}

Cette section s'est penchée sur l'étude des associations possibles entre le mariage précoce et les variables suivantes : le milieu de résidence, la région, le niveau de vie, la fréquentation scolaire passée, le niveau d'instruction, la taille du ménage, la religion et l'ethnie. Le test du Khi-deux a été utilisé pour tester les différentes associations. Il ressort que seuls la région, le niveau de vie, le fait d'avoir fréquenté l'école ou pas, la religion et l'ethnie ont un lien avec le mariage précoce.

Tableau 3.76 : Relation entre le mariage précoce et certaines caractéristiques sociodémographiques et économiques

\begin{tabular}{|c|c|c|c|}
\hline \multirow[t]{2}{*}{ Variables } & \multicolumn{2}{|l|}{ Age au mariage } & \multirow{2}{*}{$\begin{array}{l}\text { Degré de signification du } \\
\text { Khi-deux (seuil de } \\
\text { signification }=5 \% \text { ) }\end{array}$} \\
\hline & Précoce & Non précoce & \\
\hline Milieu de résidence & & & ns \\
\hline Urbain & 60,8 & 53,5 & \\
\hline Rural & 39,2 & 46,5 & \\
\hline Ensemble & 100,0 & 100 & \\
\hline Région & & & *** \\
\hline Centre-Sud & 11,7 & 19,1 & \\
\hline Est & 17,6 & 16,3 & \\
\hline Sahel & 39,2 & 8,1 & \\
\hline Centre Est & 27,5 & 19,8 & \\
\hline Centre-Nord & 3,9 & 26,7 & \\
\hline Ensemble & 100,0 & 100 & \\
\hline \multicolumn{4}{|l|}{ Niveau de vie } \\
\hline Faible & 60 & 80,8 & $* *$ \\
\hline Moyen & 40 & 19,2 & \\
\hline Elevé & 0 & 0 & \\
\hline Ensemble & 100 & $100,0 \%$ & \\
\hline \multicolumn{4}{|c|}{ Fréquentation scolaire passée } \\
\hline Oui & 70,6 & 53,5 & $* *$ \\
\hline Non & 29,4 & 46,5 & \\
\hline Ensemble & 100 & 100 & \\
\hline Niveau d'instruction & & & Ns \\
\hline Primaire & 73,3 & 52,5 & \\
\hline Secondaire & 26,7 & 47,5 & \\
\hline Ensemble & 100,0 & 100,0 & \\
\hline Taille du ménage & & & Ns \\
\hline Moins de 6 & 15,7 & 23,5 & \\
\hline 6 à 9 & 56,9 & 55,3 & \\
\hline 10 et plus & 27,5 & 21,2 & \\
\hline Ensemble & 100 & 100 & \\
\hline Religion & & & $* * *$ \\
\hline
\end{tabular}




\begin{tabular}{lrc}
\hline Musulmane & 80 & 59,3 \\
\hline Catholique & 14 & 39,5 \\
\hline Protestante & 6 & 1,2 \\
\hline Ensemble & 100 & 100 \\
\hline & & \\
\hline Ethnie & 41,2 & $66,3 \quad * * *$ \\
\hline Mossi & 29,4 & 8,1 \\
\hline Peulh & 9,8 & 4,7 \\
\hline Gourmantché & 7,8 & 14 \\
\hline Bissa & 2 & 0 \\
\hline Dioula & 9,8 & 7 \\
\hline Autre & 100 & 100 \\
\hline Ensemble & $* * *$ significatif au seuil de $1 \% ; * *$ significatif au seuil de $5 \%$; ns = non significatif
\end{tabular}



L'analyse des résultats de l'étude montre que la situation des adolescentes n'est pas reluisante. L'ampleur du mariage précoce dans la zone d'étude montre bien que le problème est toujours d'actualité. La plupart d'entre elles sont des élèves, d'où le risque de leur déscolarisation pour le mariage.

Elles connaissent très peu le Code des Personnes et de la Famille et n'ont pas d'une manière générale déjà participé à une séance de sensibilisation sur le Code. Pour celles qui sont mariées, certaines sont d'avis que le choix de leur conjoint se fait quelquefois par ellesmêmes, mais dans bien des cas ce choix est fait par les parents en accord avec la jeune fille ou par les parents seuls.

La participation de l'adolescente mariée à la prise de décision dans le foyer est très faible. Les décisions concernant les charges du ménage, la santé, l'éducation et la santé des enfants sont généralement prises par l'homme. La discussion entre mari et femme n'est pas une chose fréquente. Dans les couples où ces discussions ont lieu, les thèmes les plus couramment abordés sont la vie du couple, les grossesses, les accouchements et les mariages. Les thèmes sur la planification familiale, la santé sexuelle et reproductive, l'éducation et la scolarisation des enfants et les opportunités en matière d’emploi sont plus rarement discutés.

Elles sont parfois exposées à des conflits dont les motifs sont relatifs à la gestion du ménage, aux violences conjugales, aux soins des enfants et à la mobilité. Elles ont souvent recours aux parents de leur conjoint pour le règlement des conflits dans leur couple. Les structures telles que la Justice, l’Action Sociale sont peu sollicitées.

Pour les questions concernant la santé sexuelle et reproductive et les opportunités en matière d'emploi, la mère et le père des adolescentes constituent les principales personnes ressources qui informent les adolescentes.

Les adolescentes connaissent mal les signes d'une grossesse à risque, les conséquences d'une grossesse précoce. Elles ont très peu entendu parler de fistule obstétricale, mais semblent connaître l'importance des visites prénatales et postnatales. Bien qu'ayant une opinion favorable sur le planning familial, elles utilisent peu les méthodes contraceptives et les raisons de la non utilisation ne sont pas toujours explicites à l'exception la stérilité.

Une large proportion d'entre elles a entendu parler du Sida mais seulement très peu ont fait le test du VIH. Les adolescentes n'ont pas beaucoup utilisé les services de santé sexuelle et reproductive. Cependant, parmi celles qui ont déjà utilisé ces services, la majorité se déclare satisfaite.

En matière d'emploi, elles s'occupent essentiellement des travaux domestiques et exercent très rarement des emplois rémunérés. Elles aimeraient bien effectué des travaux domestiques rémunérés.

- La majorité d'entre elles souhaiteraient bénéficier d'une éducation ou d'une formation professionnelle. Il existe dans les régions étudiées des opportunités en matière d'éducation, mais très peu d'établissements d'enseignement technique et de formation professionnelle.

- Les améliorations souhaitées en matière de santé sexuelle et reproductive portent sur la proximité des services, la disponibilité et la réduction des coûts des médicaments, la 
qualification et l'effectif du personnel. Les besoins exprimés sont axés sur la planification familiale, le centre d'écoute pour jeunes, la vaccination, le counseling, le centre de sensibilisation. Les besoins en informations sur la santé sexuelle et reproductive s'adressent aux IST, au planning familial, aux soins pré et postnatals et à l'utilisation de la contraception.

Au regard des résultats qui découlent de l'étude, les recommandations suivantes peuvent être formulées afin de contribuer à améliorer la situation des adolescentes et à réduire l'ampleur du mariage précoce dans la zone d'étude :

- $\quad$ sensibiliser davantage les parents, l'entourage et les adolescentes sur les inconvénients du mariage précoce ;

- sensibiliser les chefs de ménage, l'entourage et les adolescentes sur le Code des personnes et de la famille ;

- appuyer financièrement les adolescentes qui souhaitent fréquenter ou poursuivre leurs études ;

- renforcer la connaissance des adolescentes sur la santé sexuelle et reproductive et mettre à leur disposition des services appropriés ;

- créer ou renforcer des structures d'accueil pour des formations professionnelles accessibles aux adolescentes ;

- faciliter l'accès des adolescentes aux crédits, les sensibiliser sur les activités génératrices de revenus et renforcer leurs capacités en matière de gestion. 


\section{BIBLIOGRAPHIE}

1. Annuaires Statistiques du MEBA (2005, 2006, 2007)

2. Cissé M., Kaboré I., Saloucou L., Mar M., Zio B. : Analyse situationnelle des services de santé de la reproduction au Burkina Faso, 2007.

3. Guiella G. : Santé Sexuelle et de la Reproduction des Jeunes au Burkina Faso : Un Etat des Lieux, Occasional Report, New York: The Alan Guttmacher Institute, 2004, No. 12.

4. Guiella G et WOOG V: Santé sexuelle et de la reproduction des adolescents au Burkina Faso : Résultats de l'Enquête Nationale sur les Adolescents du Burkina Faso 2004. Occasional Report, New York: Guttmacher Institute, 2006, No. 21.

5. Household Survey Manual: Diarrhoea Case Management, Morbidity, and Mortality, Diarrhoeal Disease Control Programme, World Health Organisation, Geneva, 1989.

6. Kaboré G, Traoré K, Lingani Y, Souloucou L (2004) ; Adolescence et vie conjugale : exemple de deux localités du Burkina Faso.

7. Recensement général de la population et de l'habitation de 2006 : Résultats définitifs ; INSD (2008)

8. Rapport de l'Enquête Démographique et de Santé du Burkina Faso 2003, INSD (2004)

9. Susheela and Renee Samara (1996), Early Mariage Among Women in Developing Countries 



\section{ANNEXES}

\section{Annexe1 : Tableaux complémentaires}

Tableau A1 : Lien de parenté des membres avec le chef de ménage

\begin{tabular}{lrrrrrr}
\hline $\begin{array}{l}\text { Lien de parenté } \\
\text { avec le chef de } \\
\text { ménage }\end{array}$ & \multicolumn{1}{c}{ Centre-Sud } & \multicolumn{1}{c}{ Est } & Sahel & Centre-Est & Centre-Nord & Total \\
\cline { 2 - 8 } CM & 14,1 & 16,0 & 13,8 & 20,1 & 15,1 & 16,1 \\
\hline Epouse/époux & 17,2 & 15,2 & 14,3 & 17,6 & 16,0 & 16,3 \\
\hline Fils/fille & 50,6 & 48,2 & 42,5 & 47,6 & 44,2 & 47,2 \\
\hline Gendre/bru & 0,2 & 0,4 & 0,1 & 0,5 & 0,3 & 0,3 \\
\hline Beau fils/fille & 1,1 & 0,3 & 1,6 & 0,6 & 1,3 & 0,9 \\
\hline Petit fils/ fille & 3,2 & 3,1 & 3,9 & 2,4 & 6,6 & 3,8 \\
\hline Père/mère & 1,7 & 1,9 & 4,1 & 1,2 & 1,7 & 1,8 \\
\hline $\begin{array}{l}\text { Beau-père/belle- } \\
\text { mère }\end{array}$ & 0,0 & 0,1 & 0,2 & 0,0 & 0,1 & 0,1 \\
\hline Frère/sœur & 3,3 & 4,1 & 6,2 & 4,4 & 3,9 & 4,1 \\
\hline Coépouse & 0,1 & 0,0 & 0,0 & 0,0 & 0,3 & 0,1 \\
\hline $\begin{array}{l}\text { Enfant de la } \\
\text { conjointe }\end{array}$ & 0,2 & 0,1 & 0,1 & 0,1 & 0,1 & 0,1 \\
\hline Autre parent & 3,2 & 2,6 & 4,7 & 2,4 & 4,4 & 3,3 \\
\hline $\begin{array}{l}\text { Sans lien de } \\
\text { parenté }\end{array}$ & 1,8 & 3,0 & 0,3 & 1,7 & 2,2 & 2,0 \\
\hline Neveu/nièce & 3,4 & 4,9 & 8,1 & 1,4 & 3,7 & 3,8 \\
\hline Total & 100,0 & 100,0 & 100,0 & 100,0 & 100,0 & 100,0 \\
\hline Effectif & 2552 & 2289 & 998 & 2424 & 2298 & 10561 \\
\hline
\end{tabular}

Tableau A2 : Répartition par sexe des membres du ménage

\begin{tabular}{lrrrrrr}
\hline Sexe & \multicolumn{7}{c}{ Région } \\
\cline { 2 - 7 } & Centre-Sud & \multicolumn{1}{c}{ Est } & \multicolumn{1}{c}{ Sahel } & Centre-Est & Centre-Nord & Centre-Sud \\
\hline Masculin & 47,7 & 48,0 & 45,5 & 47,8 & 46,3 & 47,3 \\
\hline Féminin & 52,3 & 52,0 & 54,5 & 52,2 & 53,7 & 52,7 \\
\hline Total & 100,0 & 100,0 & 100,0 & 100,0 & 100,0 & 100,0 \\
\hline Effectif & 2552 & 2289 & 998 & 2424 & 2298 & 10561 \\
\hline
\end{tabular}

Tableau A3 : Nombre de chambres à coucher dans les ménages

\begin{tabular}{|c|c|c|c|c|c|c|}
\hline \multirow{2}{*}{$\begin{array}{l}\text { Nombre de } \\
\text { chambres à } \\
\text { coucher de } \\
\text { votre } \\
\text { maison }\end{array}$} & \multicolumn{6}{|c|}{ Région } \\
\hline & Centre-Sud & Est & Sahel & Centre-Est & Centre-Nord & Total \\
\hline 1 & 10,1 & 21,3 & 9,4 & 29,1 & 8,7 & 17,7 \\
\hline 2 & 18,8 & 30,8 & 34,1 & 27,7 & 29,0 & 27,3 \\
\hline 3 & 22,2 & 26,2 & 29,0 & 22,5 & 25,8 & 24,4 \\
\hline 4 & 19,4 & 11,4 & 10,9 & 11,3 & 13,0 & 13,3 \\
\hline 5 & 14,0 & 5,4 & 8,0 & 4,5 & 9,6 & 8,0 \\
\hline 6 & 7,0 & 2,7 & 2,9 & 2,0 & 5,2 & 4,0 \\
\hline 7 ou plus & 8,4 & 2,2 & 5,8 & 2,9 & 8,7 & 5,3 \\
\hline Total & 100,0 & 100,0 & 100,0 & 100,0 & 100,0 & 100,0 \\
\hline Effectif & 356 & 367 & 138 & 488 & 345 & 1694 \\
\hline
\end{tabular}


Tableau A4 : Répartition des adolescentes selon le type d'école fréquentée (en \%)

\begin{tabular}{|c|c|c|c|c|c|c|}
\hline \multirow{2}{*}{$\begin{array}{l}\text { Type d'école } \\
\text { fréquentée }\end{array}$} & \multicolumn{6}{|c|}{ Région } \\
\hline & Centre-Sud & Est & Sahel & Centre-Est & Centre-Nord & Total \\
\hline Ecole formelle & 100,0 & 100,0 & 100,0 & 99,3 & 100,0 & 99,8 \\
\hline $\begin{array}{l}\text { Ecole non- } \\
\text { formelle }\end{array}$ & 0,0 & 0,0 & 0,0 & 0,7 & 0,0 & 0,2 \\
\hline Total & 100,0 & 100,0 & 100,0 & 100,0 & 100,0 & 100,0 \\
\hline Effectif & 189 & 211 & 71 & 295 & 203 & 969 \\
\hline
\end{tabular}

Tableau A5 : Indicateurs sur l’âge des conjoints des adolescentes (en \%)

\begin{tabular}{|c|c|c|c|c|c|c|}
\hline \multirow{2}{*}{$\begin{array}{l}\text { Age du conjoint } \\
\text { (Années) }\end{array}$} & \multicolumn{6}{|c|}{ Région } \\
\hline & Centre-Sud & Est & Sahel & Centre-Est & Centre-Nord & Total \\
\hline Mode & 20,0 & 24,0 & 22,0 & 30,0 & 25,0 & 30,0 \\
\hline Médiane & 25,0 & 24,0 & 25,0 & 30,0 & 27,0 & 26,0 \\
\hline Moyenne & 26,8 & 26,2 & 25,6 & 30,6 & 27,3 & 27,2 \\
\hline Ecart type & 8,1 & 6,2 & 3,7 & 9,3 & 4,0 & 6,7 \\
\hline Effectif & 20 & 19 & 23 & 18 & 16 & 96 \\
\hline
\end{tabular}

Tableau A6 : Niveau d'instruction des conjoints des adolescentes (en \%)

\begin{tabular}{|c|c|c|c|c|c|c|}
\hline \multirow[t]{2}{*}{ Niveau d'instruction } & \multicolumn{6}{|c|}{ Région } \\
\hline & Centre-Sud & Est & Sahel & Centre-Est & Centre-Nord & Total \\
\hline Aucun & 65,0 & 47,4 & 63,6 & 38,9 & 50,0 & 53,7 \\
\hline Primaire & 20,0 & 26,3 & 18,2 & 50,0 & 37,5 & 29,5 \\
\hline Secondaire & 15,0 & 26,3 & 18,2 & 5,6 & 12,5 & 15,8 \\
\hline Supérieur & 0,0 & 0,0 & 0,0 & 5,6 & 0,0 & 1,1 \\
\hline Total & 100,0 & 100,0 & 100,0 & 100,0 & 100,0 & 100,0 \\
\hline
\end{tabular}

Tableau A7 : Répartition des conjoints selon l’activité principale (en \%)

\begin{tabular}{|c|c|c|c|c|c|c|}
\hline \multirow[t]{2}{*}{ Activité principale } & \multicolumn{6}{|c|}{ Région } \\
\hline & Centre-Sud & Est & Sahel & Centre-Est & Centre-Nord & Total \\
\hline Aucun & 10,0 & 0,0 & 0,0 & 11,1 & 0,0 & 4,2 \\
\hline Agriculture & 60,0 & 36,8 & 68,2 & 16,7 & 31,3 & 44,2 \\
\hline Elevage & 5,0 & 0,0 & 0,0 & 0,0 & 0,0 & 1,1 \\
\hline Artisanat & 0,0 & 21,1 & 0,0 & 5,6 & 0,0 & 5,3 \\
\hline Commerce & 10,0 & 21,1 & 22,7 & 27,8 & 18,8 & 20,0 \\
\hline Elèves & 0,0 & 5,3 & 0,0 & 0,0 & 0,0 & 1,1 \\
\hline Autre & 15,0 & 15,8 & 9,1 & 38,9 & 50,0 & 24,2 \\
\hline Total & 100,0 & 100,0 & 100,0 & 100,0 & 100,0 & 100,0 \\
\hline
\end{tabular}


Tableau A8: Raisons pour l'âge souhaité au mariage chez les adolescentes

\begin{tabular}{lrrrrrr}
\hline Pourquoi cet âge? & \multicolumn{7}{c}{ Région } \\
\cline { 2 - 9 } & Centre-Sud & \multicolumn{1}{c}{ Est } & \multicolumn{1}{c}{ Sahel } & Centre-Est & Centre-Nord & Total \\
\hline Choix personnel & 77,4 & 61,0 & 65,7 & 66,2 & 71,5 & 68,8 \\
\hline Décision des parents & 0,8 & 0,8 & 0,0 & 4,8 & 0,4 & 1,7 \\
\hline Pratique courant de ma communauté & 1,9 & 1,3 & 1,0 & 9,2 & 0,9 & 3,4 \\
\hline Autre & 16,2 & 29,2 & 32,3 & 17,4 & 19,7 & 21,4 \\
\hline Ne sait pas & 3,8 & 7,6 & 1,0 & 2,4 & 7,5 & 4,7 \\
\hline Total & 100,0 & 100,0 & 100,0 & 100,0 & 100,0 & 100,0 \\
\hline Effectif & 265 & 236 & 99 & 293 & 228 & 1121 \\
\hline
\end{tabular}

Tableau A10 : Caractéristiques de murs des logements par région (\%)

\begin{tabular}{lrrrrrr}
\hline \multirow{2}{*}{ Type de murs } & \multicolumn{7}{c}{ Région } \\
\cline { 2 - 8 } & Centre-Sud & \multicolumn{1}{c}{ Est } & \multicolumn{1}{c}{ Sahel } & Centre-Est & Centre-Nord & \multicolumn{1}{c}{ Total } \\
\hline Banco & 48,5 & 18,0 & 66,7 & 30,9 & 33,6 & 35,3 \\
\hline Banco amélioré & 20,2 & 63,5 & 19,6 & 38,9 & 33,3 & 37,6 \\
\hline Ciment & 29,1 & 18,0 & 13,0 & 29,7 & 32,5 & 26,3 \\
\hline NA (Non applicable) & 1,7 & 0,0 & 0,0 & 0,2 & 0,0 & 0,4 \\
\hline Paille & 0,6 & 0,5 & 0,7 & 0,2 & 0,3 & 0,4 \\
\hline Autre & 0,0 & 0,0 & 0,0 & 0,0 & 0,3 & 0,1 \\
\hline Total & 100,0 & 100,0 & 100,0 & 100,0 & 100,0 & 100,0 \\
\hline Effectif & $\mathbf{3 5 7}$ & $\mathbf{3 6 7}$ & $\mathbf{1 3 8}$ & $\mathbf{4 8 8}$ & $\mathbf{3 4 5}$ & $\mathbf{1 6 9 5}$ \\
\hline
\end{tabular}

Tableau A11 : Caractéristiques de la toiture des logements par région (en \%)

\begin{tabular}{|c|c|c|c|c|c|c|}
\hline \multirow[t]{2}{*}{ Type de toiture } & \multicolumn{6}{|c|}{ Région } \\
\hline & Centre-Sud & Est & Sahel & Centre-Est & Centre-Nord & Total \\
\hline Banco & 2,2 & 1,4 & 61,6 & 0,2 & 1,4 & 6,1 \\
\hline Tôle ondulée & 51,5 & 78,7 & 34,1 & 81,4 & 90,4 & 72,5 \\
\hline Tôle bac & 3,4 & 2,7 & 2,9 & 2,7 & 3,8 & 3,1 \\
\hline Béton & 0,6 & 0,5 & 0,7 & 0,2 & 0,3 & 0,4 \\
\hline Paille & 42,3 & 16,6 & 0,7 & 15,6 & 4,1 & 17,9 \\
\hline Total & 100,0 & 100,0 & 100,0 & 100,0 & 100,0 & 100,0 \\
\hline Effectif & 357 & 367 & 138 & 488 & 345 & 1695 \\
\hline \multirow[t]{2}{*}{ Type de toilettes } & \multicolumn{6}{|c|}{ Région } \\
\hline & Centre-Sud & Est & Sahel & Centre-Est & Centre-Nord & Total \\
\hline Pas de toilette/nature & 34,3 & 42,6 & 16,7 & 35,2 & 8,4 & 29,6 \\
\hline WC avec chasse d'eau & 5,1 & 2,7 & 5,1 & 1,4 & 20,0 & 6,6 \\
\hline $\begin{array}{l}\text { Fosse/latrine } \\
\text { rudimentaire }\end{array}$ & 31,4 & 18,0 & 54,3 & 26,5 & 36,8 & 30,1 \\
\hline Latrine améliorée & 29,2 & 36,6 & 23,9 & 36,8 & 34,8 & 33,7 \\
\hline Total & 100,0 & 100,0 & 100,0 & 100,0 & 100,0 & 100,0 \\
\hline Effectif & 353 & 366 & 138 & 486 & 345 & 1688 \\
\hline
\end{tabular}


Tableau A13 : Principal combustible utilisé pour la cuisine par région (en \%)

\begin{tabular}{lrrrrrr}
\hline Principal combustible & \multicolumn{7}{c}{ Région } \\
\cline { 2 - 8 } & Centre-Sud & Est & Sahel & Centre-Est & Centre-Nord & Total \\
\hline Bouse & 0,6 & 0,3 & 0,7 & 0,0 & 0,3 & 0,3 \\
\hline Bois/ paille & 87,1 & 88,6 & 92,8 & 84,6 & 84,1 & 86,5 \\
\hline Charbon de bois & 0,8 & 4,6 & 1,4 & 1,6 & 2,9 & 2,4 \\
\hline Pétrole & 0,0 & 0,5 & 0,7 & 0,0 & 0,3 & 0,2 \\
\hline Gaz & 11,5 & 5,4 & 4,3 & 13,3 & 12,5 & 10,3 \\
\hline Autre & 0,0 & 0,5 & 0,0 & 0,4 & 0,0 & 0,2 \\
\hline Total & 100,0 & 100,0 & 100,0 & 100,0 & 100,0 & 100,0 \\
\hline Effectif & 357 & 367 & 138 & 488 & 345 & 1695 \\
\hline
\end{tabular}

Tableau A14 : Statut d'occupation du logement

\begin{tabular}{lrrrrrrr}
\hline $\begin{array}{l}\text { Êtes-vous } \\
\text { propriétaire de } \\
\text { votre logement ? }\end{array}$ & \multicolumn{1}{c}{ Centre-Sud } & Est & Sahel & Centre-Est & Centre-Nord & Total \\
\hline Propriétaire & 77,2 & 79,0 & 89,9 & 69,3 & 73,6 & 75,6 \\
\hline Locataire & 18,5 & 19,3 & 8,0 & 24,6 & 20,0 & 19,9 \\
\hline Autre & 4,2 & 1,6 & 2,2 & 6,1 & 6,4 & 4,5 \\
\hline Total & 100,0 & 100,0 & 100,0 & 100,0 & 100,0 & 100,0 \\
\hline Effectif & 356 & 367 & 138 & 488 & 345 & 1694 \\
\hline
\end{tabular}

Tableau A14: Personnes ressources pour les fiançailles

\begin{tabular}{lrrrrrr}
\hline $\begin{array}{l}\text { Fiançailles, } \\
\text { mariage ou vie de } \\
\text { famille ? }\end{array}$ & Centre-Sud & Est & Sahel & Centre-Est & Centre-Nord & Total \\
\hline $\begin{array}{l}\text { N'en parle à } \\
\text { personne }\end{array}$ & 34,0 & 46,2 & 14,3 & 21,0 & 38,5 & 32,3 \\
\hline Père & 3,7 & 5,2 & 2,7 & 40,1 & 7,3 & 14,6 \\
\hline Mère & 29,9 & 21,9 & 21,4 & 14,8 & 23,5 & 22,0 \\
\hline Frère & 0,3 & 0,3 & 0,9 & 1,4 & 1,9 & 1,0 \\
\hline Sour & 1,4 & 0,7 & 0,9 & 2,5 & 1,9 & 1,6 \\
\hline Mari & 3,1 & 3,1 & 0,0 & 3,9 & 2,3 & 2,9 \\
\hline Coépouse & 0,0 & 0,3 & 0,0 & 0,0 & 0,0 & 0,1 \\
\hline Amie & 21,1 & 13,9 & 43,8 & 10,9 & 14,6 & 17,4 \\
\hline Cousine & 0,7 & 0,0 & 0,0 & 0,0 & 0,4 & 0,2 \\
\hline Autre parent & 3,7 & 0,3 & 5,4 & 1,4 & 2,3 & 2,2 \\
\hline Autre parente & 0,7 & 2,1 & 10,7 & 1,1 & 1,2 & 2,1 \\
\hline Enseignant & 0,0 & 1,4 & 0,0 & 0,0 & 0,0 & 0,3 \\
\hline Employeur & 0,0 & 0,0 & 0,0 & 1,7 & 0,0 & 0,5 \\
\hline Autre & 1,4 & 4,5 & 0,0 & 1,1 & 6,2 & 2,8 \\
\hline Total & 100,0 & 100,0 & 100,0 & 100,0 & 100,0 & 100,0 \\
\hline Effectif & 295 & 288 & 116 & 361 & 260 & 1320 \\
\hline
\end{tabular}


Tableau A15 : Personnes ressources pour sur les problèmes de santé

\begin{tabular}{lrrrrrr}
\hline $\begin{array}{l}\text { Questions de } \\
\text { santé }\end{array}$ & \multicolumn{7}{c}{ Région } & \multicolumn{2}{c}{} \\
\cline { 2 - 8 } $\begin{array}{l}\text { N'en parle à } \\
\text { personne }\end{array}$ & 22,1 & 10,4 & 5,4 & 1,1 & 32,7 & 14,5 \\
\hline Père & 11,2 & 11,8 & 4,5 & 51,0 & 8,5 & 21,1 \\
\hline Mère & 37,1 & 35,1 & 22,3 & 22,7 & 23,1 & 28,7 \\
\hline Frère & 2,0 & 2,4 & 1,8 & 5,6 & 4,6 & 3,6 \\
\hline Sœur & 2,4 & 4,2 & 0,9 & 3,1 & 1,2 & 2,6 \\
\hline Mari & 5,8 & 6,3 & 7,1 & 4,8 & 4,2 & 5,4 \\
\hline Coépouse & 0,0 & 0,3 & 0,0 & 0,3 & 0,4 & 0,2 \\
\hline Amie & 8,5 & 3,8 & 35,7 & 2,5 & 5,8 & 7,6 \\
\hline Cousine & 1,0 & 0 & 0 & 0,3 & 0,0 & 0,3 \\
\hline Autre parent & 5,1 & 2,8 & 3,6 & 2,5 & 4,2 & 3,6 \\
\hline Autre parente & 1,7 & 4,9 & 10,7 & 1,7 & 1,5 & 3,1 \\
\hline Enseignant & 0,0 & 2,4 & 0,0 & 0,0 & 1,2 & 0,8 \\
\hline Employeur & 0,0 & 0,3 & 0,0 & 2,2 & 0,0 & 0,7 \\
\hline Autre & 3,1 & 15,3 & 8,0 & 2,2 & 12,7 & 7,9 \\
\hline Total & 100,0 & 100,0 & 100,0 & 100,0 & 100,0 & 100,0 \\
\hline Effectif & 294 & 288 & 112 & 357 & 260 & 1311 \\
\hline
\end{tabular}

Tableau A16: Personnes ressources pour les opportunités d'emploi

\begin{tabular}{lrrrrrr}
\hline $\begin{array}{l}\text { Opportunités } \\
\text { d'emploi }\end{array}$ & \multicolumn{7}{c}{ Région } & & \\
\cline { 2 - 7 } & Centre-Sud & Est & Sahel & Centre-Est & $\begin{array}{r}\text { Centre- } \\
\text { Nord }\end{array}$ & Total \\
\hline $\begin{array}{l}\text { N'en parle à } \\
\text { personne }\end{array}$ & 62,6 & 44,4 & 40,2 & 16,9 & 42,3 & 40,3 \\
\hline Père & 19,4 & 18,1 & 14,7 & 29,3 & 24,2 & 22,4 \\
\hline Mère & 6,5 & 6,6 & 11,8 & 14,1 & 6,9 & 9,1 \\
\hline Frère & 2,4 & 5,2 & 4,9 & 13,5 & 3,5 & 6,5 \\
\hline Sœeur & 1,4 & 1,7 & 1,0 & 3,4 & 2,3 & 2,2 \\
\hline Mari & 1,0 & 2,4 & 0,0 & 1,7 & 3,1 & 1,8 \\
\hline Coépouse & 0,0 & 0,3 & 0,0 & 0,0 & 0,0 & 0,1 \\
\hline Amie & 1,7 & 5,2 & 16,7 & 1,7 & 3,8 & 4,1 \\
\hline Cousine & 0,3 & 0,0 & 1,0 & 0,0 & 0,4 & 0,2 \\
\hline Autre parent & 1,0 & 1,7 & 2,9 & 10,7 & 4,6 & 4,7 \\
\hline Autre parente & 0,3 & 3,1 & 2,9 & 1,1 & 0,0 & 1,3 \\
\hline Enseignant & 0,7 & 8,3 & 2,9 & 5,1 & 5,8 & 4,8 \\
\hline Employeur & 0,3 & 0,0 & 0,0 & 1,7 & 0,4 & 0,6 \\
\hline Autre & 2,4 & 2,8 & 1,0 & 0,8 & 2,7 & 2,0 \\
\hline Total & 100,0 & 100,0 & 100,0 & 100,0 & 100,0 & 100,0 \\
\hline Effectif & 295 & 288 & 116 & 361 & 260 & 1320 \\
\hline
\end{tabular}

Tableau A17 : Personnes ressources pour la scolarisation

\begin{tabular}{lrrrrrr}
\hline $\begin{array}{l}\text { Opportunité de } \\
\text { scolarisation }\end{array}$ & \multicolumn{7}{c}{ Région } & & \\
\cline { 2 - 8 } & Centre-Sud & Est & Sahel & Centre-Est & Centre-Nord & \multicolumn{1}{c}{ Total } \\
\hline $\begin{array}{l}\text { N'en parle à } \\
\text { personne }\end{array}$ & 62,6 & 44,4 & 40,2 & 16,9 & 42,3 & 40,3 \\
\hline Père & 19,4 & 18,1 & 14,7 & 29,3 & 24,2 & 22,4 \\
\hline Mère & 6,5 & 6,6 & 11,8 & 14,1 & 6,9 & 9,1 \\
\hline Frère & 2,4 & 5,2 & 4,9 & 13,5 & 3,5 & 6,5 \\
\hline
\end{tabular}




\begin{tabular}{lrrrrrr}
\hline Sœur & 1,4 & 1,7 & 1,0 & 3,4 & 2,3 & 2,2 \\
\hline Mari & 1,0 & 2,4 & 0,0 & 1,7 & 3,1 & 1,8 \\
\hline Coépouse & 0,0 & 0,3 & 0,0 & 0,0 & 0,0 & 0,1 \\
\hline Amie & 1,7 & 5,2 & 16,7 & 1,7 & 3,8 & 4,1 \\
\hline Cousine &, 3 & & 1,0 & & 4 &, 2 \\
\hline Autre parent & 1,0 & 1,7 & 2,9 & 10,7 & 4,6 & 4,7 \\
\hline Autre parente &, 3 & 3,1 & 2,9 & 1,1 & & 1,3 \\
\hline Enseignant &, 7 & 8,3 & 2,9 & 5,1 & 5,8 & 4,8 \\
\hline Employeur &, 3 & & & 1,7 & 0,4 &, 6 \\
\hline Autre & 2,4 & 2,8 & 1,0 &, 8 & 2,7 & 2,0 \\
\hline Total & 100,0 & 100,0 & 100,0 & 100,0 & 100,0 & 100,0 \\
\hline Effectif & 295 & 288 & 116 & 361 & 260 & 1320 \\
\hline
\end{tabular}

Tableau A18 : Personnes ressources pour la gestion des conflits

\begin{tabular}{lrrrrrr}
\hline $\begin{array}{l}\text { Gestion de } \\
\text { conflits }\end{array}$ & \multicolumn{7}{c}{ Région } & & \\
\cline { 2 - 8 } & Centre-Sud & \multicolumn{1}{c}{ Est } & Sahel & Centre-Est & Centre-Nord & \multicolumn{1}{c}{ Total } \\
\hline $\begin{array}{l}\text { N'en parle à } \\
\text { personne }\end{array}$ & 43,5 & 25,4 & 27,3 & 1,1 & 58,1 & 29,5 \\
\hline Père & 15,6 & 5,6 & 2,7 & 28,9 & 5,0 & 13,8 \\
\hline Mère & 15,0 & 31,0 & 31,8 & 24,2 & 17,7 & 23,0 \\
\hline Frère & 0,0 & 2,8 & 1,8 & 10,7 & 1,9 & 4,1 \\
\hline Sœur & 0,3 & 3,5 & 0,9 & 4,2 & 1,2 & 2,3 \\
\hline Mari & 2,7 & 2,8 & 0,9 & 5,1 & 2,3 & 3,1 \\
\hline Coépouse & 0,0 & & 0,0 & 0,3 & 0,0 & 0,1 \\
\hline Amie & 14,3 & 12,9 & 24,5 & 5,3 & 5,8 & 10,7 \\
\hline Cousine & 0,0 & & 0,0 & 0,3 & 0,0 & 0,1 \\
\hline Autre parent & 7,1 & 2,1 & 2,7 & 12,1 & 2,3 & 6,0 \\
\hline Autre parente & 0,0 & 5,6 & 7,3 & 1,7 & 0,4 & 2,4 \\
\hline Enseignant & 0,0 & 0,3 & 0,0 & 0,3 & 0,0 & 0,2 \\
\hline Employeur & 0,0 & 0,0 & 0,0 & 3,7 & 0,4 & 1,1 \\
\hline Autre & 1,4 & 8,0 & 0,0 & 2,2 & 5,0 & 3,7 \\
\hline Total & 100,0 & 100,0 & 100,0 & 100,0 & 100,0 & 100,0 \\
\hline Effectif & 295 & 288 & 116 & 361 & 260 & 1320 \\
\hline
\end{tabular}


Tableau A19: Utilisation, type et lieu d'approvisionnement de méthodes contraceptives et les raisons de sa non utilisation chez les adolescentes

\begin{tabular}{|c|c|c|c|c|c|c|}
\hline \multirow{2}{*}{$\begin{array}{l}\text { Utilisation de méthode } \\
\text { contraceptive }\end{array}$} & \multicolumn{6}{|c|}{ Région } \\
\hline & Centre-Sud & Est & Sahel & Centre-Est & Centre-Nord & Ensemble \\
\hline Non & 41,3 & 46,6 & 71,7 & 71,5 & 49,0 & 56,7 \\
\hline Oui & 58,7 & 53,4 & 28,3 & 28,5 & 51,0 & 43,3 \\
\hline Total & 100,0 & 100,0 & 100,0 & 100,0 & 100,0 & 100,0 \\
\hline Effectif & 110 & 106 & 95 & 148 & 105 & 564 \\
\hline \multicolumn{7}{|l|}{$\begin{array}{l}\text { Type de méthode } \\
\text { utilisée }\end{array}$} \\
\hline Pilule & 4,7 & 1,8 & 19,2 & 4,7 & 9,4 & 6,6 \\
\hline$\overline{D I U}$ & 0,0 & 0,0 & 0,0 & 0,0 & 0,0 & 0,0 \\
\hline Norplant & 1,6 & 3,6 & 3,8 & 2,3 & 3,8 & 2,9 \\
\hline Injection & 4,7 & 7,1 & 19,2 & 7,0 & 7,5 & 7,9 \\
\hline Condom & 59,4 & 67,9 & 53,8 & 79,1 & 69,8 & 66,5 \\
\hline Spermicide & 0,0 & 1,8 & 0,0 & 4,7 & 0,0 & 1,2 \\
\hline Ogino/calendrier & 0,0 & 7,1 & 15,4 & 0,0 & 1,9 & 3,7 \\
\hline Retrait & 0,0 & 0,0 & 0,0 & 2,3 & 0,0 & 0,4 \\
\hline $\begin{array}{l}\text { Gris-gris ou méthode } \\
\text { traditionnelle }\end{array}$ & 0,0 & 0,0 & 0,0 & 2,3 & 0,0 & 0,4 \\
\hline Abstinence & 31,3 & 17,9 & 0,0 & 11,6 & 13,2 & 17,4 \\
\hline Collier & 0,0 & 0,0 & 0,0 & 0,0 & 3,8 & 0,8 \\
\hline Effectif & 65 & 58 & 29 & 45 & 54 & 251 \\
\hline \multicolumn{7}{|l|}{$\begin{array}{l}\text { Raisons de la non } \\
\text { utilisation de la } \\
\text { contraception }\end{array}$} \\
\hline $\begin{array}{l}\text { Manque } \\
\text { d'information/source }\end{array}$ & 4,8 & 0,0 & 1,3 & 13,2 & 1,1 & 5,0 \\
\hline $\begin{array}{l}\text { Difficulté d'accès aux } \\
\text { méthodes }\end{array}$ & 0,0 & 1,7 & 1,3 & 12,3 & 0,0 & $\overline{4,0}$ \\
\hline Religion & 1,6 & 5,0 & 5,3 & 2,6 & 2,3 & 3,2 \\
\hline $\begin{array}{l}\text { Opposition du } \\
\text { partenaire } \\
\end{array}$ & 3,2 & 5,0 & 2,6 & 4,4 & 3,4 & 3,7 \\
\hline $\begin{array}{l}\text { Crainte des effets } \\
\text { secondaire }\end{array}$ & 0,0 & 1,7 & 0,0 & 7,0 & 3,4 & 3,0 \\
\hline N'approuve pas le PF & 0,0 & 0,0 & 0,0 & 0,9 & 0,0 & 0,2 \\
\hline $\begin{array}{l}\text { Veux avoir une } \\
\text { grossesse }\end{array}$ & 1,6 & 5,0 & 10,5 & 3,5 & 3,4 & 4,7 \\
\hline $\begin{array}{l}\text { Actuellement en } \\
\text { grossesse }\end{array}$ & 4,8 & 6,7 & 6,6 & 5,3 & 3,4 & 5,2 \\
\hline Partenaire absent & 0,0 & 1,7 & 7,9 & 0,9 & 1,1 & 2,2 \\
\hline Allaitement & 4,8 & 1,7 & 3,9 & 4,4 & 0,0 & 3,0 \\
\hline Autres & 52,4 & 67,2 & 55,8 & 64,9 & 44,3 & 57,1 \\
\hline Effectif & 45 & 48 & 66 & 103 & 51 & 313 \\
\hline \multicolumn{7}{|l|}{$\begin{array}{l}\text { Lieu } \\
\text { d'approvisionnement }\end{array}$} \\
\hline Pharmacie & 20,3 & 63,6 & 26,9 & 66,7 & 27,8 & 39,2 \\
\hline Centre de santé & 7,8 & 13,6 & 34,6 & 5,1 & 11,1 & 12,3 \\
\hline Marché & 3,1 & 11,4 & 0,0 & 0,0 & 1,9 & 3,5 \\
\hline Boutique & 31,3 & 9,1 & 3,8 & 20,5 & 25,9 & 20,7 \\
\hline Mari & 1,6 & 0,0 & 0,0 & 0,0 & 1,9 & 0,9 \\
\hline Autres & 35,9 & 2,3 & 34,6 & 7,7 & 31,5 & 23,3 \\
\hline Total & 100,0 & 100,0 & 100,0 & 100,0 & 100,0 & 100,0 \\
\hline Effectif & 65 & 58 & 29 & 45 & 54 & 251 \\
\hline
\end{tabular}


Tableau A20 : Connaissance d'un endroit où se procurer le préservatif

\begin{tabular}{|c|c|c|c|c|c|c|}
\hline \multirow{2}{*}{$\begin{array}{l}\text { Savez-vous où se } \\
\text { procurer des } \\
\text { préservatifs? }\end{array}$} & \multicolumn{6}{|c|}{ Région } \\
\hline & Centre-Sud & Est & Sahel & Centre-Est & Centre-Nord & Ensemble \\
\hline Non & 8,6 & 14,3 & 7,7 & 0,0 & 5,7 & 7,7 \\
\hline Oui & 91,4 & 82,9 & 92,3 & 100,0 & 91,4 & 90,9 \\
\hline NSP & 0,0 & 2,9 & 0,0 & 0,0 & 2,9 & 1,4 \\
\hline Total & 100,0 & 100,0 & 100,0 & 100,0 & 100,0 & 100,0 \\
\hline Effectif & 34 & 35 & 17 & 27 & 35 & 148 \\
\hline \multicolumn{7}{|l|}{ Quel endroit? } \\
\hline $\begin{array}{l}\text { Pharmacie/dépôt } \\
\text { pharmaceutique }\end{array}$ & 46,9 & 69,0 & 91,7 & 72,0 & 68,8 & 66,2 \\
\hline Centre de santé & 3,1 & 13,8 & 83,3 & 20,0 & 12,5 & 18,5 \\
\hline Marché & 21,9 & 27,6 & 8,3 & 8,0 & 9,4 & 16,2 \\
\hline Boutique & 87,5 & 44,8 & 75,0 & 64,0 & 93,8 & 73,8 \\
\hline$\overline{\text { Effectif }}$ & 34 & 35 & 17 & 27 & 35 & 148 \\
\hline
\end{tabular}

Tableau A21 : Présence d'un centre d'alphabétisation dans les localités

\begin{tabular}{lrrrrrr}
\hline $\begin{array}{l}\text { Présence de centre } \\
\text { d'alphabétisation }\end{array}$ & \multicolumn{7}{c}{ Région } \\
\cline { 2 - 7 } & Centre-Sud & \multicolumn{1}{c}{ Est } & Sahel & Centre-Est & Centre-Nord & Ensemble \\
\hline Oui & 53,2 & 74,0 & 91,1 & 41,2 & 55,4 & 58,0 \\
\hline Non & 46,8 & 26,0 & 8,9 & 58,8 & 44,6 & 42,0 \\
\hline Total & 100,0 & 100,0 & 100,0 & 100,0 & 100,0 & 100,0 \\
\hline Effectif & 295 & 288 & 116 & 361 & 260 & 1320 \\
\hline
\end{tabular}

Tableau A22 : Centre d'alphabétisation accessible aux adolescentes dans leur localité

\begin{tabular}{|c|c|c|c|c|c|c|}
\hline \multirow{2}{*}{$\begin{array}{l}\text { Centre d'alpha } \\
\text { accessible aux } \\
\text { adolescentes }\end{array}$} & \multicolumn{6}{|l|}{ Région } \\
\hline & Centre-Sud & Est & Sahel & Centre-Est & Centre-Nord & Ensemble \\
\hline$\overline{\text { Oui }}$ & 91,1 & 98,3 & 100,0 & 96,3 & 88,7 & 94,7 \\
\hline Non & 8,9 & 1,7 & & 3,7 & 11,3 & 5,3 \\
\hline Total & 100,0 & 100,0 & 100,0 & 100,0 & 100,0 & 100,0 \\
\hline Effectif & 295 & 288 & 116 & 361 & 260 & 1320 \\
\hline
\end{tabular}

Tableau A23 : Présence de centres de formation professionnelle dans les localités

\begin{tabular}{lrrrrrr}
\hline $\begin{array}{l}\text { Présence de centre } \\
\text { de formation } \\
\text { professionnelle }\end{array}$ & \multicolumn{1}{c}{ Centre-Sud } & \multicolumn{1}{c}{ Est } & Sahel & Centre-Est & Centre-Nord & Ensemble \\
\hline Oui & 32,8 & 40,2 & 43,2 & 30,4 & 41,7 & 36,4 \\
\hline Non & 45,7 & 29,7 & 26,1 & 25,9 & 28,2 & 31,7 \\
\hline NSP & 21,5 & 30,1 & 30,6 & 43,7 & 30,1 & 32,0 \\
\hline Total & 100,0 & 100,0 & 100,0 & 100,0 & 100,0 & 100,0 \\
\hline Effectif & 295 & 288 & 116 & 361 & 260 & 1320 \\
\hline
\end{tabular}


Tableau A24 : Type de formation professionnelle dans les localités

\begin{tabular}{lrrrrrrr}
\hline $\begin{array}{l}\text { Quel type de formation } \\
\text { professionnelle ? }\end{array}$ & \multicolumn{7}{c}{ Région } \\
\cline { 3 - 9 } & Centre-Sud & \multicolumn{1}{c}{ Est } & Sahel & Centre-Est & Centre-Nord & Ensemble \\
\hline Coiffeuse/couture & 61,7 & 69,8 & 83,0 & 55,7 & 73,8 & 67,2 \\
\hline Gestion/commerce & 8,5 & 19,0 & 4,3 & 27,4 & 10,3 & 15,3 \\
\hline Artisanat/poterie & & 4,3 & 3,4 & 6,4 & 17,9 & 12,1 & 9,1 \\
\hline $\begin{array}{l}\text { Fabrication } \\
\text { alimentaires }\end{array}$ & produits & 1,1 & 0,9 & 14,9 & 6,6 & 5,6 & 4,7 \\
\hline Autre & & 13,7 & 52,6 & 17,0 & 16,3 & 15,0 & 24,5 \\
\hline Effectif & 97 & 116 & 50 & 110 & 108 & 480 \\
\hline
\end{tabular}

Tableau A25 : Fréquence des biens ou équipements possédés par les ménages selon le niveau de vie

\begin{tabular}{llrrr}
\hline Variables & Modalité & \multicolumn{3}{c}{ Niveau de vie } \\
\cline { 3 - 5 } & & Pauvre & Moyen & \multicolumn{2}{c}{ Riche } \\
\hline Possession de radio & & $68,3 \%$ & $81,3 \%$ & $100,0 \%$ \\
\hline Possession de télévision & & $7,3 \%$ & $12,5 \%$ & $50,0 \%$ \\
\hline Possession de téléphone & & $31,7 \%$ & $62,5 \%$ & $100,0 \%$ \\
\hline Electricité & & $0,0 \%$ & $0,0 \%$ & $50,0 \%$ \\
\hline Possession de Mobylette & & $4,9 \%$ & $50,0 \%$ & $100,0 \%$ \\
\hline $\begin{array}{l}\text { Principal moyen de } \\
\text { transport }\end{array}$ & Vélo & $92,7 \%$ & $56,3 \%$ & $50,0 \%$ \\
\hline \multirow{nyyyy}{*}{ Type de murs } & Mobylette/moto & $2,4 \%$ & $37,5 \%$ & $50,0 \%$ \\
\cline { 2 - 5 } & Banco & $73,2 \%$ & $43,8 \%$ & $0,0 \%$ \\
\cline { 2 - 5 } & Banco amélioré & $26,8 \%$ & $50,0 \%$ & $50,0 \%$ \\
\cline { 2 - 5 } & Ciment & $0,0 \%$ & $6,3 \%$ & $50,0 \%$ \\
\hline Type de toilettes & Pas de toilette/nature & $65,9 \%$ & $12,5 \%$ & $0,0 \%$ \\
\cline { 2 - 5 } & Fosse/latrine rudimentaire & $31,7 \%$ & $43,8 \%$ & $50,0 \%$ \\
\cline { 2 - 5 } & Latrine améliorée & $0,0 \%$ & $0,0 \%$ & $50,0 \%$ \\
\hline
\end{tabular}

Graphique A1 : Présence de conflits dans les couples

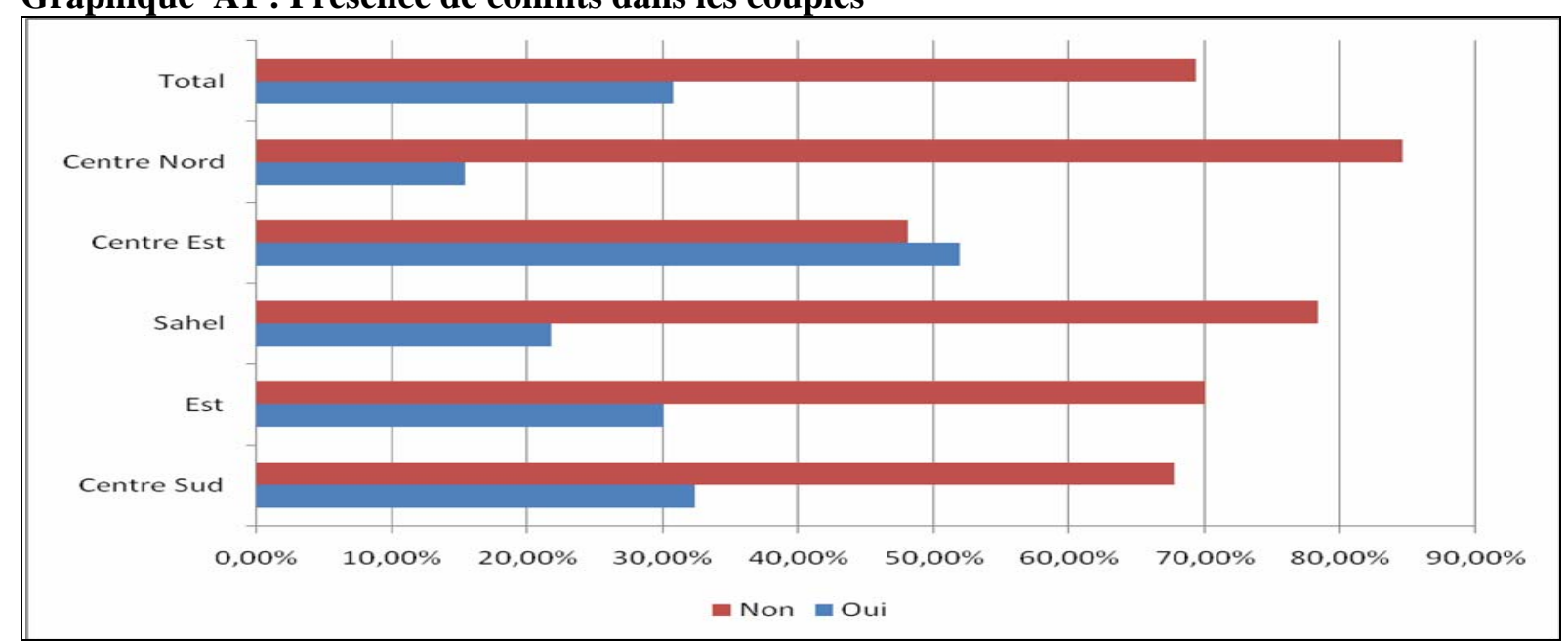

